NORTHWESTERN UNIVERSITY

NONLINEAR RESONANCE ISLANDS AND MODULATIONAL EFFECTS IN A PROTON SYNCHROTRON

\author{
A DISSERTATION \\ SUBMITTED TO THE GRADUATE SCHOOL \\ IN PARTIAL FULFILLMENT OF THE REQUIREMENTS \\ for the degree \\ DOCTOR OF PHILOSOPHY
}

Field of Physics and Astronomy

By

Todd J. Satogata

EVANSTON, ILLINOIS

February 1993 
c Copyright by Todd J. Satogata 1993

All Rights Reserved 


\title{
ABSTRACT \\ NONLINEAR RESONANCE ISLANDS AND MODULATIONAL EFFECTS IN A PROTON SYNCHROTRON
}

\author{
Todd J. Satogata
}

We examine both one-dimensional and two-dimensional nonlinear resonance islands created in the transverse phase space of a proton synchrotron by nonlinear magnets. We also examine application of the theoretical framework constructed to the phenomenon of modulational diffusion in a collider model of the Fermilab Tevatron.

For the one-dimensional resonance island system, we examine the effects of two types of modulational perturbations on the stability of these resonance islands: tune modulation and beta function modulation. Hamiltonian models are presented which predict stability boundaries that depend on only three parameters: the strength and frequency of the modulation and the frequency of small oscillations inside the resonance island. These models are compared to particle tracking with excellent agreement. The tune modulation model is also successfully tested in experiment, where frequency domain analysis coupled with tune modulation is demonstrated to be useful in measuring the strength of a nonlinear resonance.

Nonlinear resonance islands are also examined in two transverse dimensions in the presence of coupling and linearly independent crossing resonances. We present a first-order Hamiltonian model which predicts fixed point locations, but does not reproduce small oscillation frequencies seen in tracking; therefore in this circumstance such a model is inadequate. Particle tracking is presented which shows evidence of two-dimensional persistent signals, and we make suggestions on methods for observing such signals in future experiment. 
Finally, we apply the tune modulation stability diagram to the explicitly twodimensional phenomenon of modulational diffusion in the Fermilab Tevatron with beam-beam kicks as the source of nonlinearity. We find that the amplitude growth created by this mechanism in simulation is exponential rather than root-time as predicted by modulational diffusion models. Finally, we comment upon the luminosity and lifetime limitations such a mechanism implies in a proton storage ring. 


\section{ACKNOWLEDGEMENTS}

I would like to thank the members of my thesis committee, Professons Bruno Gobbi, John Ketterson and in particular Robert Oakes, for their patience and understanding. Their valuable commentary at the dissertation defense improved this document greatly over its original version.

Graduate school is not without tense moments, and many people made even those moments bright with their presence. In particular, Marco Fatuzzo and Salah Chaurize, each housemates who were always there in a pinch; Brian O'Reilly, whose roguish charm and conversation were a delight; Kevin Mcllhany, who was always there to keep me up late asking the right questions and refusing the wrong answers; and Scott Kalbfeld, a smiling face who put up with my appearing only once a month for lunch. I would also like to graciously thank the members of the departmental administration, especially Chris Lopes, for enduring the antics of one of their wayward brethren.

I have made many fine friends not only in personal experience, but across the widening expanse of computer networks such as the Internet. The diversity of minds and their willingness to share new visions and cast new light on old have made me more complete than I was before. Scott, Deb, Juli, Doug, Jeannine, and above all, Sandra, were always there with their unflagging support and understanding.

I first learned of the existence of the field of accelerator physics from Leo Michelotti in a course taught many years ago at Northwestern. To him in particular I owe a debt of gratitude for his tutelage whenever I had a technical questions about dynamics. The Accelerator Physics group at Fermilab hosted me during this research, and a more interactive and dynamic group of physicists and engineers I have never seen. The comments and companionship of Jim Holt, Bill Gabella, Jian-Ping Shan and especially François Ostiguy made my stay with them 
rewarding.

The E778 collaboration includes many fine people as well as outstanding physicists, and I am honored and grateful to have been counted among their company. In particular Ben Cole, Dick Talman, Lia Meriminga and George Tsironis were always there with ideas, with directions and with heart.

Above all the rest of my academic associates there is my advisor and my friend, Steve Peggs. Without his patience, his insight, his humor and his energy, this dissertation would be nothing but a sparkle and a dream.

Finally, I must offer my heartfelt love and thanks to my parents, Blythe and Frank, from who I learned so much about a lifetime of wonderment, and to my precious wife Sue, with whom I continue to share this amazing journey. They are all, in their own ways, constant inspirations. 


\section{TABLE OF CONTENTS}

ABSTRACT Tii

ACKNOWLEDGEMENTS v v

TABLE OF CONTENTS vii

LIST OF FIGURES ix

Chapter

1. INTRODUCTION 1

2. ACCELERATOR PHYSICS FUNDAMENTALS

2.1 Accelerator Coordinate Systems 5

2.2 Transverse Linear Motion in a Synchrotron 9

2.3 Generating Functions and Canonical Transformation $\quad 15$

$\begin{array}{ll}\text { 2.4 Linear Longitudinal Motion and Chromaticity } & 18\end{array}$

3 NONLINEAR RESONANCE ISLANDS AND ONE-DIMENSIONAL $\begin{array}{ll}\text { PERSISTANT SIGNALS } & 21\end{array}$

3.1 The First Order Nonlinear One-Turn Hamiltonian 21

3.2 The One-Dimensional Nonlinear N-Turn Hamiltonian 28

3.3 Parameterization and Character of Resonant Motion 30

3.4 First Order Nonlinear Tracking and Simulation 33

3.5 Measurements in Real Accelerators 38

4 PERTURBATIONS OF NONLINEAR RESONANCES - TUNE

MODULATION AND BETA MODULATION 45

4.1 Sources of Tune Modulation and Beta Modulation 46

4.2 The N-Turn Hamiltonian for Tune Modulated

One-Dimensional Resonances $\quad 49$

4.3 Structure of the $\left(q, Q_{M}\right)$ Parameter Space 50

4.4 The N-Turn Hamiltonian for Beta Modulated 
4.5 Comparing Hamiltonian Results to Simulation 62

5. TUNE MODULATION AND EXPERIMENT E778 68

5.1 Requirements for the Experiment $\quad 70$

5.2 Local Preparation for the Experiment $\quad 74$

5.3 The Experimental Run 81

5.4 Data Analysis and Results 85

6. PERSISTENT SIGNALS IN TWO TRANSVERSE DIMENSIONS 97

$\begin{array}{ll}\text { 6.1 Theoretical Predictions } & 97\end{array}$

6.2 Tracking of Two-Dimensional Resonance Islands $\begin{array}{ll}\text { and Persistent Signals } & 102\end{array}$

$\begin{array}{ll}\text { 6.3 A Possible Experiment } & 110\end{array}$

7. MODULATIONAL DIFFUSION 113

$\begin{array}{ll}\text { 7.1 Characteristics of Modulational Diffusion } & 114\end{array}$

7.2 The Tevatron Situation and an Operational Model 117

$\begin{array}{lr}7.3 \text { Simulation Results } & 120\end{array}$

7.4 Conclusions and Future Directions $\quad 126$

$\begin{array}{lr}\text { 8. CONCLUDING REMARKS } & 129\end{array}$

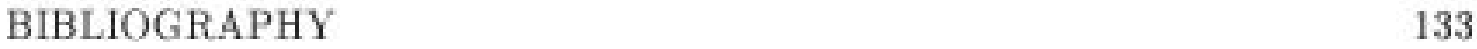




\section{LIST OF FIGURES}

Figure

page

2.1 - Local coordinate system for a synchrotron.

2.2 - Phase space ellipse transformation.

2.3 - Sample turn-by-turn Poincaré plot from tracking.

2.4 - The generating function mnemonic square.

3.1 - Isolated $5 Q_{x}$ resonance islands in various coordinate systems.

3.2 - A simple lattice for nonlinear kick projection.

3.3 - The octupole-decapole simulation lattice.

3.4 - Island tune scaling with octupole strength, theory versus tracking.

3.5 - Island tune scaling with decapole strength, theory versus tracking.

3.6 - Decoherence of a beam kicked into nonresonance phase space.

3.7 - Motion of a small beam kicked into a nonlinear resonance island.

3.8 - Motion of a large beam kicked to overlap a nonlinear resonance island. 43

3.9 - Sample single BPM turn-by-turn data from E778, showing decoherence.

3.10 - Sample single BPM turn-by-turn dta from E778, showing resonant capture.

4.1 - The $\left(q, Q_{M}\right)$ parameter plane for tune modulation with $N=5$.

4.2 - Phase spaces at various points in the $\left(q, Q_{M}\right)$ parameter plane.

4.3 - The $\left(\Delta \beta / \beta, Q_{M}\right)$ parameter plane for beta modulation with $N=5$.

4.4 - Odfp simulation of the $\left(q, Q_{M}\right)$ stability curves for tune modulation.

4.5 - Odfp simulation of the $\left(\Delta \beta / \beta, Q_{M}\right)$ stability curves for beta modulation. 66

5.1 - 1991 E778 lattice, showing sextupole, kicker and BPM locations.

5.2 - Block diagram of the E778 data acquisition system.

5.3 - Camel graphical user interface for LeCroy 6810 control. 
5.4 - DC QXR quadrupole calibration of tune shift versus current.

5.5 - Modulated QXR quadrupole voltage calibration with AC current.

5.6 - Phase space at E17 kicker, with sextupole configuration 91.0.

5.7 - Phase space at E17 kicker, with sextupole configuration 911 .

5.8 - Voltage vs. amplitude E17 kicker calibration.

5.9 - Sample decoherent turn-by-turn data.

5.10 - Sample $Q_{x}=2 / 5$ resonant turn-by-turn data.

5.11 - Sample BPM turn-by-turn data for tune modulated persistent signal decay, showing sudden decay.

5.12 - Systematic tune modulation plane scans.

5.13 - Variation of tune with amplitude for sextupole configuration 91_0, including predictions from tracking.

5.14 - Variation of tune with amplitude for sextupole configuration 91_1, including predictions from tracking.

5.15 - Persistent signal decay in the frequency domain, for 91.0 configuration. $0 \mathrm{~Hz}<Q_{M}<50 \mathrm{~Hz}$, with $q=0.0002$.

5.16 - Same as Figure 4.13, except for $50 \mathrm{~Hz}_{2}<Q_{M}<100 \mathrm{~Hz}$.

5.17 - Same as Figure 4.13, except for $100 \mathrm{~Hz}<Q_{M}<150 \mathrm{~Hz}$.

5.18 - Same as Figure 4.13, except for $150 \mathrm{~Hz}<Q_{M}<200 \mathrm{~Hz}$.

5.19 - Same as Figure 4.13, except for $200 \mathrm{~Hz}<Q_{M}<250 \mathrm{~Hz} . \quad 92$

5.20 - Same as Figure 4.13, except for $250 \mathrm{~Hz}<Q_{M}<300 \mathrm{~Hz}$.

5.21 - Same as Figure 4.13, except for $300 \mathrm{~Hz}<Q_{M}<350 \mathrm{~Hz}$.

5.22 - Same as Figure 4.13, except for $0 \mathrm{~Hz}_{z}<Q_{M}<350 \mathrm{~Hz}$.

5.23 - Persistent signal decay rate for chirps in Figures 4.13-20, including decay rate from simulation.

5.24 - Tune modulation parameter plane $\left(q: Q_{M}\right)$, showing results for 91_0 configuration with $Q_{1}=6.3 \times 10^{-3}$. 
6.1 -Comparison of two-dimensional octupole detuning to tracking.

6.2 - The tune plane for the octupole-decapole lattice, showing first order decapole resonances and second-order octupole resonances.

6.3 - Four-dimensional phase space projections of a two-dimensional persistent signal.

6.4 - Magnified view of motion very near a four-dimensional fixed-point. $\quad 108$

6.5 - Two-dimensional island tunes versus decapole strength, from tracking. 110

7.1 - One-dimensional regular and chaotic motion.

7.2 - Resonances and scales for modulational diffusion.

7.3 - The tune plane for typical Fermilab 1992 collider operations.

7.4 - Maximum vertical amplitudes over 10 and 100 synchrotron periods, tracked with the Tevatron collider lattice.

7.5 - Same as Figure 6.4, tracked over 1000 and 10000 synchrotron periods. 122

7.6 - Character of vertical amplitude growth.

7.7 - Exponential vertical amplitude growth rates versus scaled distance to nearest coupling resonance. 


\section{CHAPTER 1 INTRODUCTION}

High energy physics, the study of matter at its most fundamental observed level, is divided into two general classes of research: theory and experiment. The drive of the theorist is to condense explanations of a broad class of phenomena to a simple model. The experimentalist must deal with existing technologies and abilities, seeking to observe exceedingly rare "events" in an attempt to reconcile our observations of nature with theory. These two work hand in hand, providing physicists with a progressively more coherent, cohesive and cogent understanding of the way nature works.

Experimental high energy physics accomplishes its task with particle accelerators, devices which raise the energies of particles such as protons and electrons, and in exotic cases, antiprotons, muons and other species, and collide them with other particles. (Accelerators also are used for other applications such as radiotherapy and coherent gamma ray production.) The objective is to create and observe events that occur at high energies, such as production of massive highly unstable particles (such as the top quark or the Higgs boson) or events that signal the effects of exceedingly weak processes (such as CP violation observed in B-meson systems).

Because these events are so weak, the number of events produced per unit time per unit cross section must be high in order to provide reasonable statistics for experimentalists. This is measured in terms of a quantity called the luminosity, a quantity depending on the frequency $f$ with which bunches of particles interact, the number $N$ of particles in each bunch and the transverse beam size $\sigma$. For 
round beams such as those at the Fermilab Tevatron,

$$
L=\frac{f N^{2}}{4 \pi \sigma^{2}}
$$

The current maximum luminosity achieved with a hadron collider, $L=8.97 \cdot 10^{30}$ $\mathrm{cm}^{2} \mathrm{~s}^{-1}$, was achieved at the Tevatron in April, 1993. Any effect that removes particles from the beam (reducing $N$ ) or increases the transverse size of the beam $\sigma$ reduces the luminosity, or the efficiency of the accelerator in producing events of interest to high energy physicists.

Particle motion around an accelerator is approximately, but not completely, linear. Even without magnetic field and alignment errors (always present at the $10^{-4}$ level) the presence of sextupoles commonly used to correct the chromaticity also introduces nonlinear kicks. Nonlinearities, therefore, cannot be removed they must be understood and corrected if their presence adversely affects accelerator performance and operations. The focus of this thesis is on effects, called resonances, driven by these nonlinearities - how their strengths can be measured, and how they might interact with modulations existing within the accelerator to affect the stability of particles in a storage ring or collider.

The study of the long-term stability of particles traveling around one of these devices is also a study of fundamental issues in classical dynamics, dating back to Poincaré's investigation of the long-term stability of the solar system in the late 19 th century. Coincidentally, typical timescales are roughly the same order of magnitude for both systems: the solar system has existed in its present form for a few billion years $\left(10^{9}\right.$ Earth orbits), and protons and antiprotons are stored in the Fermilab Tevatron for a few billion revolutions between beam dumps and refills. In order to prevent luminosity degradation in a collider over this time, the mechanisms responsible for growth of transverse particle oscillations about the central orbit over timescales up to billions of turns must be investigated. Such 
mechanisms fall into three categories — slow, medium and fast.

Fast amplitude growth is typically caused by severe distortions of the orbit, either by intense magnetic field errors (such as a reversed-polarity corrector or strong nonlinear fields) or by a major fault condition such as blockage of the beam pipe. Here the timescales for particle loss, either at the blockage or at the physical aperture, range from fractions of a turn to turns. Such losses are usually easy to diagnose with beam position and loss monitors, and it is of particular importance to guard against such losses in machines with superconducting magnets, where these losses could easily lead to a magnet quench.

Medium timescale amplitude growth occurs over timescales ranging from tens to thousands of revolutions. This growth is characteristically driven by distortions of the particle orbit, called resonances, created by nonlinear magnetic fields. Strong isolated resonances perturb the amplitudes of the orbit, possibly leading to loss at the physical aperture in tens to hundreds of turns. If many resonances are driven, nearby resonance structures in the phase space of particle motion can overlap, causing stochastic motion with timescales of up to thousands of turns (Schoch 1958). These sorts of mechanisms and structures have been investigated in the context of resonant trapping (Chao and Month 1974, Chao et. al. 1987b) and general nonlinear dynamics (Lichtenberg and Lieberman 1983) as applied to accelerators. Experience with such systems in real machines leads to constraints on the horizontal and vertical tunes at which operation is acceptable, reasonably far from loss-creating resonances.

Slow amplitude growth occurs over much much longer timescales, from $10^{4}$ to $10^{9}$ turns. These timescales are macroscopic, seconds to hours of actual accelerator operation, and are therefore normally very difficult to diagnose. Most mechanisms that drive slow growth depend strongly on the coupled multidimensional nature of the particle motion and weak sources of stochasticity or noise. Modu- 
lational (or thick-layer) diffusion, Arnold (or thin-layer) diffusion and amplitude growth driven by weak external noise all fall into this category (Lichtenberg and Lieberman 1983). Modulational diffusion (Vivaldi 1984, Chirikov et. al. 1985) is of particular interest in this dissertation because, as the name implies, it is driven by the modulation of a parameter of the dynamical system, in this case the accelerator tune.

Chapter 2 contains a review of fundamental concepts of accelerator physics that are relevant to remainder of this dissertation. A discrete Hamiltonian approach describing one-dimensional resonance islands is described in Chapter 3, including description of the primary tracking program used to simulate particles under the influence of a single nonlinear resonance. Chapter 4 extends this analysis to include both the effects of tune modulation and beta function modulation, comparing simulation and theory with excellent agreement. The tune modulation portion of Fermilab experiment E778 is described in Chapter 5, where particles trapped by nonlinear resonance islands were observed in a real accelerator and then detrapped in a controlled way with tune modulation. Chapter 6 returns to unperturbed resonance islands, extending the one-dimensional results of Chapter 3 to two transverse dimensions, and Chapter 7 investigates modulational diffusion, a multidimensional phenomena driven by tune modulation in two transverse dimensions. 


\section{CHAPTER 2 ACCELERATOR PHYSICS FUNDAMENTALS}

This chapter describes the coordinate systems and magnet strength definitions used in the remainder of this thesis. Because a thorough background in accelerator physics is not assumed and definitions used by various researchers within the field typically vary, aspects of the field are also discussed here that are relevant to the remainder of this work. In $\S 2.1$ the local transverse coordinate system used to expand transverse motion in a synchrotron is described, as well as the definitions of both linear and nonlinear magnet strengths. In $\S 2.2$ the transverse linear dynamics of a strong-focusing synchrotron are discussed. In $\S 2.3$ the discrete Hamiltonian formalism that will be used to investigate the nonlinear dynamics of this system is introduced, and the forms of generating functions and transformations that will be applied to the discrete Hamiltonian in Chapters 3 and 4 are described; $\S 2.4$ returns to investigate the longitudinal motion in a synchrotron and how this motion couples to the transverse dimension.

Table (2.1) lists typical values of many quantities relevant to accelerator operations at the Tevatron collider, the Indiana University Cyclotron Facility (IUCF) cooling ring and the SSC collider. Many of these quantities are not mentioned here in detail, but are listed for completeness.

\subsection{ACCELERATOR COORDINATE SYSTEMS}

Typically the equilibrium orbit, or closed orbit, around a synchrotron can be approximated as a circle with a constant radius $\rho$ - this is equivalent to stating that the accelerator approximately consists of nothing but dipole magnets, ignor-

ing the effects of vertical bends and long straight sections. (For example, $75 \%$ of the Fermilab Tevatron and $80 \%$ of the Fermilab Booster circumferences consist 


\begin{tabular}{|l|c|c|c|c|}
\hline Parameter & $\begin{array}{c}\text { Symbol } \\
(\text { units })\end{array}$ & $\begin{array}{c}\text { FNAL } \\
\text { Tev }\end{array}$ & $\begin{array}{c}\text { IUCF } \\
\text { Cooler }\end{array}$ & $\begin{array}{c}\text { SSC } \\
\text { Collider }\end{array}$ \\
\hline \hline Horizontal Tune & $Q_{x}$ & 20.586 & 3.82 & 123.28 \\
Vertical Tune & $Q_{y}$ & 20.575 & 4.85 & 123.78 \\
Synchrotron Tune & $Q_{s}$ & $5.7 \cdot 10^{-4}$ & $5 \cdot 10^{-4}$ & $1.2 \cdot 10^{-3}$ \\
Revolution Freq. & $f_{\text {rev }}(\mathrm{kHz})$ & 47.7 & $10^{3}$ & 3.4 \\
Minimum Beta & $\beta^{\star}(\mathrm{m})$ & 0.5 & 1 & 1 \\
Maximum Beta & $\beta_{\max }(\mathrm{m})$ & 200 & 50 & $8 \cdot 10^{3}$ \\
Dispersion (ave. $)$ & $\eta(\mathrm{m})$ & 0.5 & 0.2 & 1 \\
Momentum Spread & $\sigma_{p} / p$ & $2 \cdot 10^{-4}$ & $4 \cdot 10^{-5}$ & $10^{-4}$ \\
Kinetic Energy & $E(\mathrm{GeV})$ & 900 & 0.045 & $2 \cdot 10^{4}$ \\
Rigidity & $B \rho(\mathrm{T}-\mathrm{m})$ & $3 \cdot 10^{3}$ & 3.6 & $7 \cdot 10^{4}$ \\
Bend Radius & $\rho(\mathrm{m})$ & $7.5 \cdot 10^{2}$ & 1.2 & $1.0 \cdot 10^{4}$ \\
\hline
\end{tabular}

Table 2.1: Various operational accelerator parameters for the Fermilab Tevatron collider (1993 collider lattice), the IUCF cooling storage ring and the SSC collider (Design Report). For the colliders the values given refer to a single beam.

of dipole magnets.) All transverse motion is expanded in the transverse displacements from this equilibrium closed orbit, with $\hat{x}$ being defined in the outward radial direction. The direction of beam travel on the closed orbit is defined as $\hat{s}$, always tangential to the closed orbit; then $\hat{y} \equiv-\hat{x} \times \hat{s}$ and the triplet $(\hat{x}, \hat{y}, \hat{s})$ forms a right-handed coordinate system. This agrees with the convention used in the MAD 8.1 and TEAPOT lattice design and tracking programs (Grote and Iselin 1990, Schachinger and Talman 1985), as well as that of Edwards and Syphers (Edwards and Syphers 1987).

In such a coordinate system, the magnetic dipole bending field $\vec{B}_{0}$ for a positively charged particle is oriented in the $+\hat{y}$ direction; this makes it natural to speak of a field error as being positive if it points in this direction. For a typical proton synchrotron with normal temperature dipoles this magnetic field can be as high as 2 Tesla; with superconducting dipoles such as those used in RHIC, the 

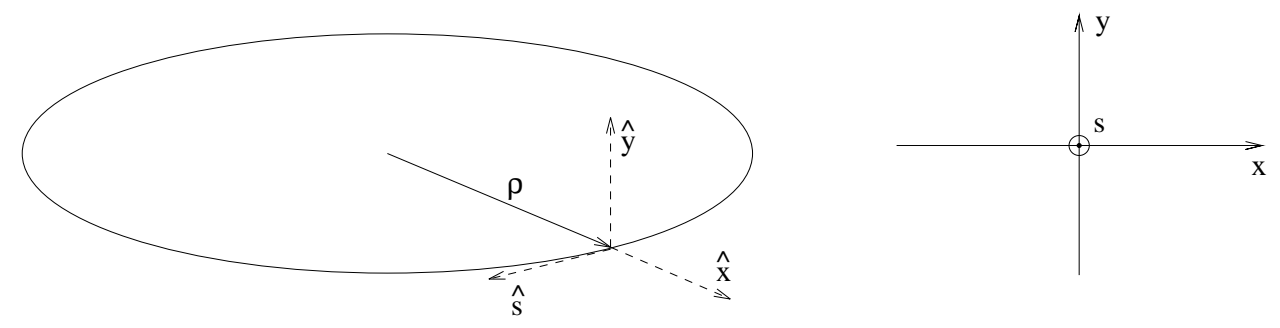

Figure 2.1: The local coordinate system in a synchrotron.

CERN LHC, the Tevatron and the SSC, $B_{0}$ can be as high as 6.6 Tesla. Magnetic fields and field errors can now be analytically expanded by

$$
i \Delta B_{x}+\Delta B_{y}=B_{0} \sum_{n=0}^{\infty}\left(b_{n}+i a_{n}\right)(x+i y)^{n},
$$

where the $b_{n}$ denote multipole strengths of normal field components and the $a_{n}$ denote multipole skew field components. The units of these multipole strengths are $m^{-n}$, and the strengths themselves range from $.3 m^{-2}$ for sextupole correctors in the Tevatron to approximately $10^{-4} \mathrm{~m}^{-n}$ for higher order multipole errors from fringe fields or magnet coil misalignments.

This field expansion agrees with the multipole strength conventions used in TEAPOT, but does not agree with the conventions used by MAD 8.1, which instead uses a Taylor expansion for the magnetic field on the midplane of the magnet, $y=0$. A comparison of the two definitions for normal multipole strengths gives the relationship

$$
b_{n}(\text { thesis })=b_{n}(\text { TEAPOT })=\frac{B_{n}(\mathrm{MAD})}{n ! B_{0}}=\frac{1}{n ! B_{0}}\left(\frac{\partial^{n} B_{y}}{\partial x^{n}}\right)_{y=0} .
$$

Typically magnetic multipole strengths are measured from angular fourier analysis of field strengths for a distribution of angular positions at a constant radius from the magnet center bore.

In this dissertation we make the normally realistic approximation that kicks from nonlinear magnetic fields are small and applied over a negligible magnet 
length $L$, or that the transverse momentum change from the nonlinearity is small compared to the total momentum of the kicked particle. The following discussion is therefore only relevant for kicks applied over short distances such as those from dipole fringe fields, quadrupoles and correctors; it does not trivially apply to multipole errors within long dipoles. The small-amplitude kicks over a short multipole are given by

$$
\Delta x^{\prime}=-\frac{L}{|B \rho|} \Delta B_{y}, \quad \Delta y^{\prime}=\frac{L}{|B \rho|} \Delta B_{x} .
$$

Here $|B \rho|$ is the magnetic rigidity, related to the particle's total momentum $p$ and charge $e$ by $|B \rho|=p / e$; units are such that $|B \rho| \approx 3.3357 p c$ for a particle with electron or proton charge when $p c$ is expressed in $\mathrm{GeV}$ and $B \rho$ in $\mathrm{T}$ - $\mathrm{m}$. The prime denotes differentiation with respect to the longitudinal coordinate $s$, so $x^{\prime} \equiv d x / d s$. We then have

$$
\Delta x^{\prime}-i \Delta y^{\prime}=-\frac{B_{0} L}{|B \rho|} \sum_{n=0}^{\infty}\left(b_{n}+i a_{n}\right)(x+i y)^{n} .
$$

For the $n=0$ case (the primary dipole bending field) $b_{0}=1$ in the horizontal plane and the quantity $B_{0} L /|B \rho|$ is the bend angle for each main dipole.

In the case of a normal quadrupole, for example, $n=1$ and

$$
\Delta x^{\prime}-i \Delta y^{\prime}=-\frac{B_{0} L}{|B \rho|} b_{1}(x+i y)
$$

For a positive normal quadrupole strength $b_{1}$ a particle displaced positively in the $\hat{x}$ direction receives a negative (focusing) kick. In the $\hat{y}$ direction, however, a positively displaced particle is given a positive (defocusing) kick.

In this thesis dispersive effects are ignored except for a few comments about sources of beta modulation in Chapter 4 - particle energies are held constant and there is no variation in the rigidity $|B \rho|$ which affects relative field strengths. For simplicity in the notation we therefore absorb the leading term (the dipole bend 
angle) into the multipole strength terms and rewrite the multipole kick expansion $(2.4)$ as

$$
\Delta x^{\prime}-i \Delta y^{\prime}=-\sum_{n=1}^{\infty}\left(\tilde{b}_{n}+i \tilde{a}_{n}\right)(x+i y)^{n}
$$

where $(\tilde{b}, \tilde{a})_{n} \equiv(b, a)_{n} B_{0} L /|B \rho|$. These are called the normalized multipole strengths and have the units $m^{-n}$.

\subsection{TRANSVERSE LINEAR MOTION IN A SYNCHROTRON}

By far the most influential paper in accelerator physics has been that of Courant and Snyder (1958), which laid the foundations for much of the field. There the transverse linear motion near the closed orbit of an alternating-gradient synchrotron was shown to be parameterized by a pair of quantities, $\beta(s)$ and $\alpha(s) \equiv$ $-\beta^{\prime}(s) / 2$, for each transverse plane of oscillation. With the dispersion function $\eta(s)$ (see Equation (2.33)) these parameters are commonly referred to as the lattice functions of the accelerator.

The "beta function" $\beta(s)$ has units of length — it ranges from approximately half a meter to 200 meters in the Tevatron collider lattice. As will be shown

shortly, the amplitude of transverse particle oscillations scales with $\sqrt{\beta(s)}$ in motion around the accelerator. At the interaction regions of many colliders, a low-beta insertion is designed to lower the beta function at the beam crossing point, thus reducing the actual beam size and increasing the luminosity.

Motion of particles in the two transverse planes is coupled even in the linear approximation by a variety of perturbations such as longitudinal solenoidal fields from experimental detectors, normal dipole and quadrupole rotation errors, vertical dipole bends and deliberately installed skew quadrupoles. With the assumption that the accelerator under consideration is flat and that there are no significant solenoidal fields, this coupling is usually small and treated perturba- 
tively. Such a treatment is followed in this thesis, and allows the linear motion in each of the transverse planes to be treated as independent.

In either transverse dimension the motion of a particle through the straight drift sections, dipoles and quadrupoles of a synchrotron is described by Hill's equation,

$$
x^{\prime \prime}(s)+K(s) x(s)=0,
$$

where $K(s)$ the focusing strength in that plane, piecewise continuous and periodic over one revolution of the machine. If the synchrotron has a superperiodicity, $K(s)$ naturally also has this superperiodicity - however, this symmetry is normally broken by low-beta insertions or other practical necessities.

Because Hill's equation is so similar to the equation of motion of a harmonic oscillator, it is typically solved by substituting a harmonic solution where both the amplitude and phase depend on $s$ :

$$
\begin{aligned}
x(s) & =\sqrt{2 J \beta(s)} \cos \psi(s) \\
& =a(s) \cos \psi(s) .
\end{aligned}
$$

The choice of normalization here is motivated by transformations derived in the next section, where $J$ is shown to be the action canonical to the phase $\psi(s)$. Substitution of this ansatz into Equation (2.7) gives two differential relations for $\psi(s)$ and $\beta(s):$

$$
\begin{aligned}
\frac{d}{d s}\left(\beta \psi^{\prime}\right) & =0, \\
2 \beta^{\prime \prime} \beta-\left(\beta^{\prime}\right)^{2}-4 \beta^{2}\left(\psi^{\prime}\right)^{2}+4 \beta^{2} K(s) & =0 .
\end{aligned}
$$

Equation (2.9) can be integrated immediately, using the standard convention that chooses the constant of integration as one, to find the integrated phase,

$$
\psi(s)=\int_{0}^{s} \frac{d S}{\beta(S)} .
$$


Similarly the phase advance over any section of the ring $\left(s_{1}, s_{2}\right)$ can be defined:

$$
\Delta \psi=\int_{s_{1}}^{s_{2}} \frac{d S}{\beta(S)}
$$

With this $\psi^{\prime}$, Equation (2.10) for the betatron function $\beta(s)$ takes the standard forms:

$$
\begin{gathered}
2 \beta^{\prime \prime} \beta-\left(\beta^{\prime}\right)^{2}+4 \beta^{2} K(s)=4, \\
1+\alpha^{\prime} \beta+\alpha^{2}=\beta^{2} K(s) .
\end{gathered}
$$

With periodic boundary conditions and piecewise continuous $K(s)$ this equation can be solved numerically to give the betatron function $\beta$ as a function of $s$.

The tune $Q$ in each plane is defined as the long-term average number of transverse betatron oscillations executed in that plane in each traversal of the ring, or the average transverse oscillation frequency divided by the revolution frequency of the machine. It is found in the linear approximation by taking the total phase advance over one traversal and dividing by $2 \pi$ :

$$
Q \equiv \frac{1}{2 \pi} \oint d S / \beta(S)
$$

Generally the solution to any linear second-order differential equation such as Equation (2.7) is uniquely determined by the initial conditions of $x$ and $x^{\prime}$. It has been shown (Courant and Snyder 1958) that the linear motion in each plane over any section $\left(s_{1}, s_{2}\right)$ of the ring can be completely described by

$$
\left(\begin{array}{c}
x \\
x^{\prime}
\end{array}\right)_{2}=M_{21}\left(\begin{array}{c}
x \\
x^{\prime}
\end{array}\right)_{1}
$$

where

$$
M_{21}=\left(\begin{array}{cc}
\sqrt{\frac{\beta_{2}}{\beta_{1}}}\left(\cos \Delta \psi+\alpha_{1} \sin \Delta \psi\right) & \sqrt{\beta_{1} \beta_{2}} \sin \Delta \psi \\
\frac{-\left(1+\alpha_{1} \alpha_{2}\right) \sin \Delta \psi-\left(\alpha_{2}-\alpha_{1}\right) \cos \Delta \psi}{\sqrt{\beta_{1} \beta_{2}}} & \sqrt{\frac{\beta_{1}}{\beta_{2}}}\left(\cos \Delta \psi-\alpha_{2} \sin \Delta \psi\right)
\end{array}\right) .
$$

The same mapping also holds for motion in the $y$ plane, using lattice functions and phase advances there. The linear transformation matrix $M_{21}$ is a combination 
of rotations and scalings which map the particles around a rotation on an ellipse in the phase space $\left(x, x^{\prime}\right)$; it is unwieldy in this basis because the parameters $\beta$ and $\psi$ are more natural for a circular coordinate system as will be shown in the next section.

The transformation matrix $M_{21}$ is actually a concatenation of several individual transformation matrices which can be found by solving simpler versions of Hill's equation. For the case where $K$ is zero, as is true in straight drift sections and approximately true in dipoles of length $L$,

$$
M_{21}(K=0)=\left(\begin{array}{cc}
1 & L \\
0 & 1
\end{array}\right)
$$

For the case where $K$ is a nonzero constant, as in a quadrupole of length $L$,

$$
M_{21}(K=\text { constant })=\left(\begin{array}{cc}
\cos (L \sqrt{K}) & \frac{1}{\sqrt{K}} \sin (L \sqrt{K}) \\
-\sqrt{K} \sin (L \sqrt{K}) & \cos (L \sqrt{K})
\end{array}\right)
$$

Keeping the integrated magnet strength $K L=\tilde{b}_{1}$ constant while taking the thin magnet limit $L \rightarrow 0$ here gives the transformation matrix of a thin horizontal quadrupole kick,

$$
M_{21}(\text { thin quad })=\left(\begin{array}{cc}
1 & 0 \\
-\tilde{b_{1}} & 1
\end{array}\right)
$$

Since a horizontally focusing quadrupole defocuses equally in the vertical direction (see Equation (2.5)), reversing the sign of $\tilde{b}_{1}$ in the above mapping gives the corresponding thin quadrupole kick in the vertical plane.

For the subjects examined in this dissertation, motion is only observed once per accelerator turn in a Poincaré surface of section; in this approach, the transverse phase space is observed in discrete time steps instead of continuously around the ring. This method of visualization is more practical in application, because beam position monitors and other diagnostic equipment measure beam properties on a turn-by-turn basis. The longitudinal coordinate $s$ is transformed to an integer 

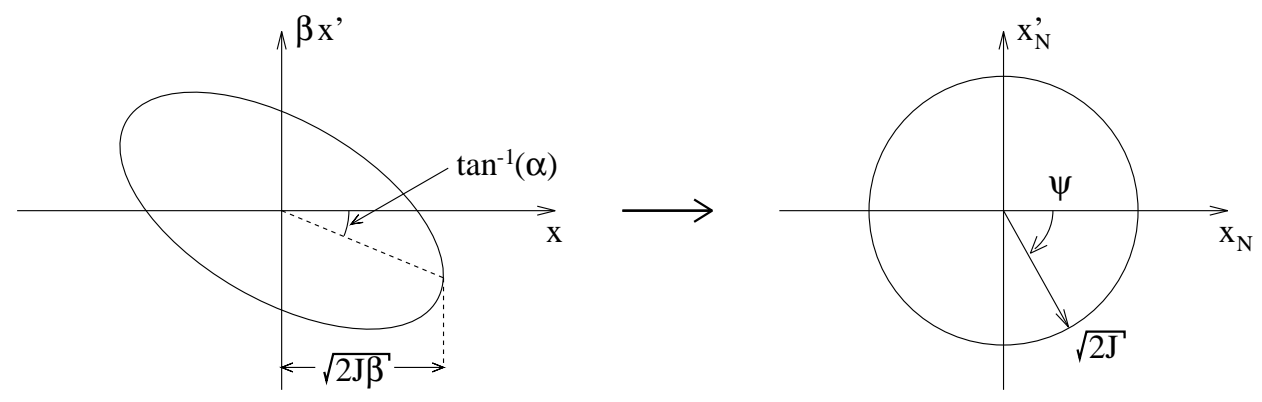

Figure 2.2: The phase space ellipse transformation (2.23) from physical transverse coordinates $\left(x, x^{\prime}\right)$ to normalized transverse coordinates $\left(x_{N}, x_{N}^{\prime}\right)$. The physical amplitude of betatron oscillations at a point with beta function $\beta$ is $a=\sqrt{2 J \beta}$.

turn-number coordinate $t \equiv[s / 2 \pi \rho]$ so the mapping represented by Equations (2.15) and (2.16) now becomes a "one-turn map":

$$
\left(\begin{array}{c}
x \\
x^{\prime}
\end{array}\right)_{t+1}=M\left(\begin{array}{c}
x \\
x^{\prime}
\end{array}\right)_{t}
$$

where the linear one-turn transformation matrix is

$$
M=\left(\begin{array}{cc}
\cos (2 \pi Q)+\alpha \sin (2 \pi Q) & \beta \sin (2 \pi Q) \\
-\frac{\left(1+\alpha^{2}\right)}{\beta} \sin (2 \pi Q) & \cos (2 \pi Q)-\alpha \sin (2 \pi Q)
\end{array}\right)
$$

and the lattice functions $\beta$ and $\alpha$ are those of the observation point. It is also interesting to note that imposing a small thin quadrupole kick on the one-turn map gives the tune shift associated with a quadrupole error of strength $\tilde{b}_{1}$ as

$$
\Delta Q_{x, y}(\mathrm{quad})= \pm \frac{\tilde{b}_{1} \beta(\mathrm{quad})}{4 \pi}
$$

with the positive sign referring to the horizontal plane and the negative to the vertical. 


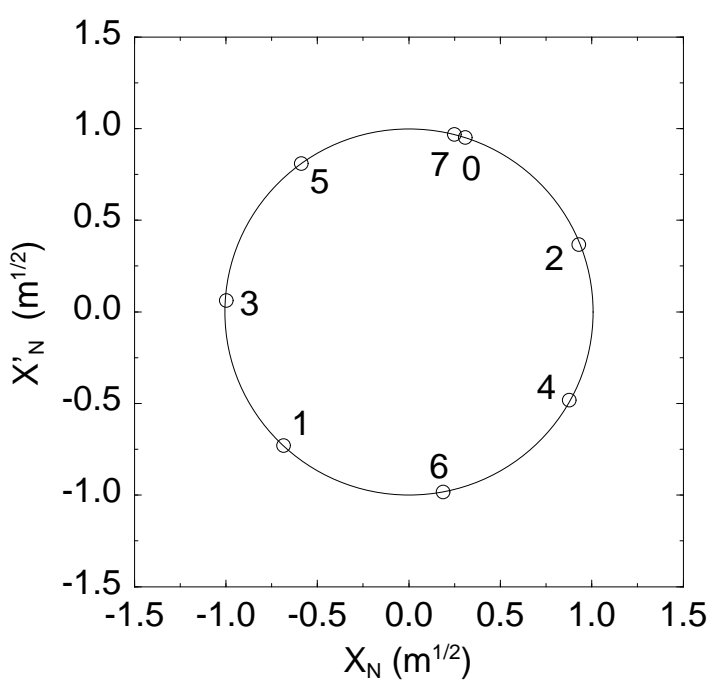

Figure 2.3: Seven turns of a linear one-dimensional turn-by-turn Poincaré plot in normalized coordinates $\left(x_{N}, x_{N}^{\prime}\right)$, with tune $Q=.57$ near the $4 Q=7$ resonance. This is simulation of a single particle following the linear one-turn map of Equation (2.20); each successive point lies on a circular contour.

A coordinate transformation exists that transforms the elliptical mapping of Equation (2.21) into a circular one, so that linear motion consists of a rotation around this circle. One transformation to these "normalized coordinates" $\left(x_{N}, x_{N}^{\prime}\right)$ is given by

$$
\begin{aligned}
x(s) & =\sqrt{\beta(s)} x_{N}(s), \\
x^{\prime}(s) & =\frac{1}{\sqrt{\beta(s)}} x_{N}^{\prime}(s)-\frac{\alpha(s)}{\sqrt{\beta(s)}} x_{N}(s) .
\end{aligned}
$$

Both $x_{N}$ and $x_{N}^{\prime}$ have units of $m^{1 / 2}$ as do all distances in the normalized coordinate system. After this transformation both $M_{12}$ and $M$ become pure rotation matrices, rotating in the clockwise direction in the $\left(x_{N}, x_{N}^{\prime}\right)$ plane by angles of $\Delta \psi$ and $2 \pi Q$ respectively. Both $\alpha$ and $\beta$ are functions of the longitudinal coordinate $s$, but due to the periodicity of the lattice functions they are independent of $t$ and thus so is the one-turn mapping. 
Because linear phase space motion is nothing more than a rotation, if the rotation angle is a rational multiple of $2 \pi$ (or the tune $Q$ is rational), the particle returns to the same phase space position in some number of turns. A perturbation at a particular location in the accelerator having the same periodicity in the phase angle will always kick the particle in the same way, possibly creating amplitude growth, luminosity degradation and particle loss. Generalizing to both transverse dimensions, the resonance condition on the horizontal and vertical tunes is given by

$$
j Q_{x}+k Q_{y}=l
$$

where $j, k$ and $l$ are integers. The perturbations mentioned above, periodic in the phase, drive these resonances - they are caused by nonlinear forces such as nonlinear magnet errors and magnetic forces felt by particles comprising colliding beams (the beam-beam force). Following (2.24), resonances have nonlinear kicks with a phase dependence $\cos \left(j \psi_{x}+k \psi_{y}\right)$.

\subsection{GENERATING FUNCTIONS AND CANONICAL TRANSFORMATIONS}

Hill's equation, Equation (2.7), is also the equation of motion for a harmonic oscillator with a time-dependent restoring force and no damping. A nonautonomous Hamiltonian can be written for such an oscillator:

$$
H\left(x, p_{x} ; s\right)=\frac{p_{x}^{2}}{2}+K(s) \frac{x^{2}}{2},
$$

where $p_{x}$ is the canonical momentum associated with the position $x$. Application of Hamilton's equations immediately gives $p_{x}=x^{\prime}$, so the coordinate $x^{\prime}$ is the canonical conjugate of the position $x$. This Hamiltonian can be canonically transformed to the form of an action-angle harmonic oscillator with the time-dependent type 1 generating function (Goldstein 1980, Chapter 9):

$$
G_{1}\left(\left[x, x^{\prime}\right] \rightarrow[\psi, J] ; s\right)=-\frac{x^{2}}{2 \beta(s)}[\tan \psi(s)+\alpha(s)] .
$$


This nontrivially produces the coordinate transformations and new Hamiltonian

$$
\begin{aligned}
x & =\sqrt{2 J \beta} \cos \psi(s), \\
x^{\prime} & =-\sqrt{2 J / \beta}[\sin \psi(s)+\alpha(s) \cos \psi(s)], \\
H(\psi, J ; s) & =H\left(x, x^{\prime} ; s\right)+\frac{d G_{1}}{d s}=\frac{J}{\beta(s)} .
\end{aligned}
$$

This is exactly the same as the harmonic solution, Equation (2.8), of Hill's equation mentioned previously, but this approach also identifies the appropriate canonical coordinates for a Hamiltonian analysis of the linear problem.

Motion will be expressed in a "discrete" Hamiltonian formalism throughout this thesis, where one-turn and N-turn maps similar to the linear map of Equation (2.20) are generated from the discrete Hamiltonian $H$ and the corresponding discrete forms of Hamilton's equations:

$$
\frac{\Delta x_{N}}{\Delta t}=\frac{\partial H}{\partial x_{N}^{\prime}}, \quad \frac{\Delta x_{N}^{\prime}}{\Delta t}=-\frac{\partial H}{\partial x_{N}} .
$$

The linear one-turn motion over the ring is reproduced if the linear Hamiltonian (2.27) is integrated over one ring revolution. Then the discrete Hamiltonian and one-turn discrete equations of motion for the canonical coordinates $(\psi, J)$ are:

$$
\begin{gathered}
H_{1}(\psi, J)=2 \pi Q J, \\
\Delta \psi=2 \pi Q, \\
\Delta J=0 .
\end{gathered}
$$

Since the beta function is periodic around the ring, the linear one-turn Hamiltonian $H_{1}$ is independent of turn number if the tune of the synchrotron is constant.

In order for the discrete forms of Hamilton's equation to retain their form when coordinate transformations are applied to the Hamiltonian, it is necessary and sufficient to require these transformations to be canonical. In this dissertation a variety of (possibly time-dependent) generating functions are used to generate these canonical transformations (Goldstein 1980, Landau and Lifshitz 1975). 


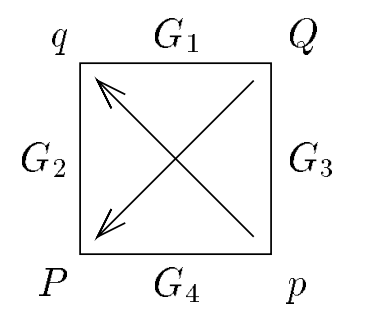

$$
\begin{array}{lll}
G_{1}(q, Q): & p=\partial G_{1} / \partial q & P=-\partial G_{1} / \partial Q \\
G_{2}(q, P): & p=\partial G_{2} / \partial q & Q=\partial G_{2} / \partial P \\
G_{3}(p, Q): & q=-\partial G_{3} / \partial p & P=-\partial G_{3} / \partial Q \\
G_{4}(p, P): & q=-\partial G_{4} / \partial p & Q=\partial G_{4} / \partial P
\end{array}
$$

Figure 2.4: The generating function mnemonic square. The generating functions $G_{i}$ are functions of the coordinates that bracket them, and partial derivatives in the direction of the arrows are positive. The Hamiltonian is also changed by an amount $d G_{i} / d t$ in the transformation, if the generating function is time-dependent.

There are four common types of canonical transformations from coordinates $(q, p)$ to $(Q, P)$, imaginatively named type 1 through type 4 . For example, the type 1 generating function used above depends only on the old and new coordinates $q$ and $Q$, not on the momenta $p$ and $P$; it gives the transformation equations

$$
\begin{aligned}
p & =\frac{\partial G_{1}(q, Q ; t)}{\partial q}, \\
P & =-\frac{\partial G_{1}(q, Q ; t)}{\partial Q}, \\
H(q, p ; t) & \rightarrow H(Q, P ; T)+\frac{d G_{1}(q, Q ; t)}{d t} .
\end{aligned}
$$

The four types of generating functions and their respective transformation equations may be conveniently summarized using a mnemonic square similar to that used in thermodynamics, as shown in Figure (2.4). All linear transformations, time dependent or not, are canonical - when these are used the coordinate transformations will be stated for clarity. Other types of canonical transformations applied here are stated explicitly by their generating functions.

At several places in this thesis, discrete Hamiltonia are "integrated" (or, more properly, summed) to give equations of motion over timescales of $N$ turns, where $N$ is an integer. This requires rescaling the time coordinate; however the product of the Lagrange action and time used to derive Hamilton's equations from a least- 
action principle (Goldstein 1980, p. 364),

$$
d \mathcal{L} \equiv[p \dot{q}-H(p, q)] d t
$$

remains invariant under this transformation if the Hamiltonian is scaled to suit

the time change. Since this is implicit in the summation process, the only change necessary for such a transformation to respect the difference forms of Hamilton's equations is the rescaling of the time coordinate, $t \rightarrow N t \equiv T$.

\subsection{LONGITUDINAL MOTION AND CHROMATICITY}

In the context of the stability of transverse motion, longitudinal dynamics are important because they provide a mechanism for tune modulation to exist in every machine which uses RF systems to longitudinally stabilize and accelerate particle beams. The parameter used to quantify the coupling of the fractional momentum offset to the transverse tune in each plane is called the chromaticity $\xi_{i}$; it is defined by

$$
\Delta Q_{i}=\xi_{i} \delta
$$

in each plane. Here $\delta \equiv \Delta p / p_{0}$ is the fractional momentum offset from the ideal design momentum $p_{0}$. European convention differs significantly in definition of the chromaticity, using the fractional tune shift $\Delta Q / Q$ instead of $\Delta Q$. Chromatic effects arise from the momentum dependence of the focusing strength of quadrupoles - if a particle has a larger energy than the design energy, it is focused less strongly and executes a smaller number of betatron oscillations in one machine revolution. For a simple uncorrected alternating-gradient synchrotron the chromaticity is roughly equal in magnitude and opposite in sign to the tune.

For most machines such a large net chromaticity is undesirable, as particles with even small fractional momentum offsets can experience tune shifts large enough to shift them onto undesirable resonances. Chromaticity is adjusted with the 
addition of correction sextupoles at points of nonzero dispersion in each plane, since dispersion is the coupling between momentum offset and transverse position; for example, horizontal dispersion gives

$$
x(\text { total })=x(\text { closed orbit })+\eta_{x} \frac{\Delta p}{p_{0}} .
$$

Examining Equation (2.6) for a normal sextupole $(n=2)$ and including the effect of dispersion in the horizontal plane to first order in momentum offset, we find that sextupoles give kicks linear in displacement, or momentum-dependent quadrupole kicks:

$$
\Delta x^{\prime}-i \Delta y^{\prime}=-\tilde{b}_{2}\left[(x+i y)^{2}+2 \frac{\Delta p}{p} \eta_{x}(x+i y)\right] .
$$

For maximum efficiency a normal chromaticity correction sextupole would be placed at a point with high horizontal dispersion. Using the tune shift from a quadrupole kick, Equation (2.22), one can immediately find the contribution to the total chromaticity from a distribution of normal sextupoles:

$$
\begin{gathered}
\xi_{x}=\frac{1}{2 \pi} \sum_{i} \eta_{x}\left(s_{i}\right) \beta_{x}\left(s_{i}\right) \tilde{b}_{2}\left(s_{i}\right) \\
\xi_{y}=-\frac{1}{2 \pi} \sum_{i} \eta_{x}\left(s_{i}\right) \beta_{y}\left(s_{i}\right) \tilde{b}_{2}\left(s_{i}\right) .
\end{gathered}
$$

It is also important to note that though sextupoles can correct the chromaticity, they also introduce nonlinear kicks and transverse coupling.

Particles with momentum offsets subject to RF focusing also execute synchrotron oscillations, where $\delta$ is not constant but varies sinusoidally as though it were being modulated. Although investigation of the longitudinal phase space of a particle is complicated (Edwards and Syphers 1987, chapter 2), for longitudinal beam distributions much smaller than the RF bucket size it is a good approximation that all particles have their momentum offsets oscillating at the same frequency, the synchrotron frequency $Q_{s}$. In the absence of explicit tune 
modulation (as was used in E778) and strong power supply ripple, this is the source of tune modulation that motivates the long-term stability inquiries raised in later chapters. 


\section{CHAPTER 3 \\ NONLINEAR RESONANCE ISLANDS AND ONE-DIMENSIONAL PERSISTENT SIGNALS}

In this chapter a discrete Hamiltonian approach to two-dimensional transverse nonlinear resonances is presented that gives perturbative results exact to first order in nonlinear magnet strength. One-turn and N-turn Hamiltonians are derived from a projection map (Peggs 1982) in §3.1-2 respectively; these Hamiltonians can be considered to respectively generate the one-turn and N-turn Poincaré surface of section maps. Certain phase space structures, called "resonance islands", can appear with one-dimensional resonant motion as pictured in Figure (3.1). These structures are shown to be parameterized by several experimentally accessible quantities including the island tune, $Q_{\mathrm{I}}$, in $\S 3.3$; a computational single particle tracking simulation is described in $\S 3.4$ and compared to theoretical prediction for the $5 Q_{x}$ resonance. Various methods used to experimentally investigate nonlinear resonance islands are discussed in $\S 3.5$, in particular those used in experiment CE22 at the IUCF Cooling Ring and for this thesis in experiment E778 at Fermilab.

\subsection{THE FIRST ORDER NONLINEAR ONE-TURN HAMILTONIAN}

In the previous chapter the action-angle parameterization was presented for the uncoupled linear transverse one-turn particle motion in a synchrotron. The next issue is how to include the effects of nonlinear magnets to form a discrete Hamiltonian that more adequately describes motion in a real accelerator. A perturbative approach that is correct to first order in nonlinear magnet strengths is used here; however, there exist a multitude of other methods, both perturbative (Michelotti 1986a) and non-perturbative (Gabella 1991), that give similarly-structured 


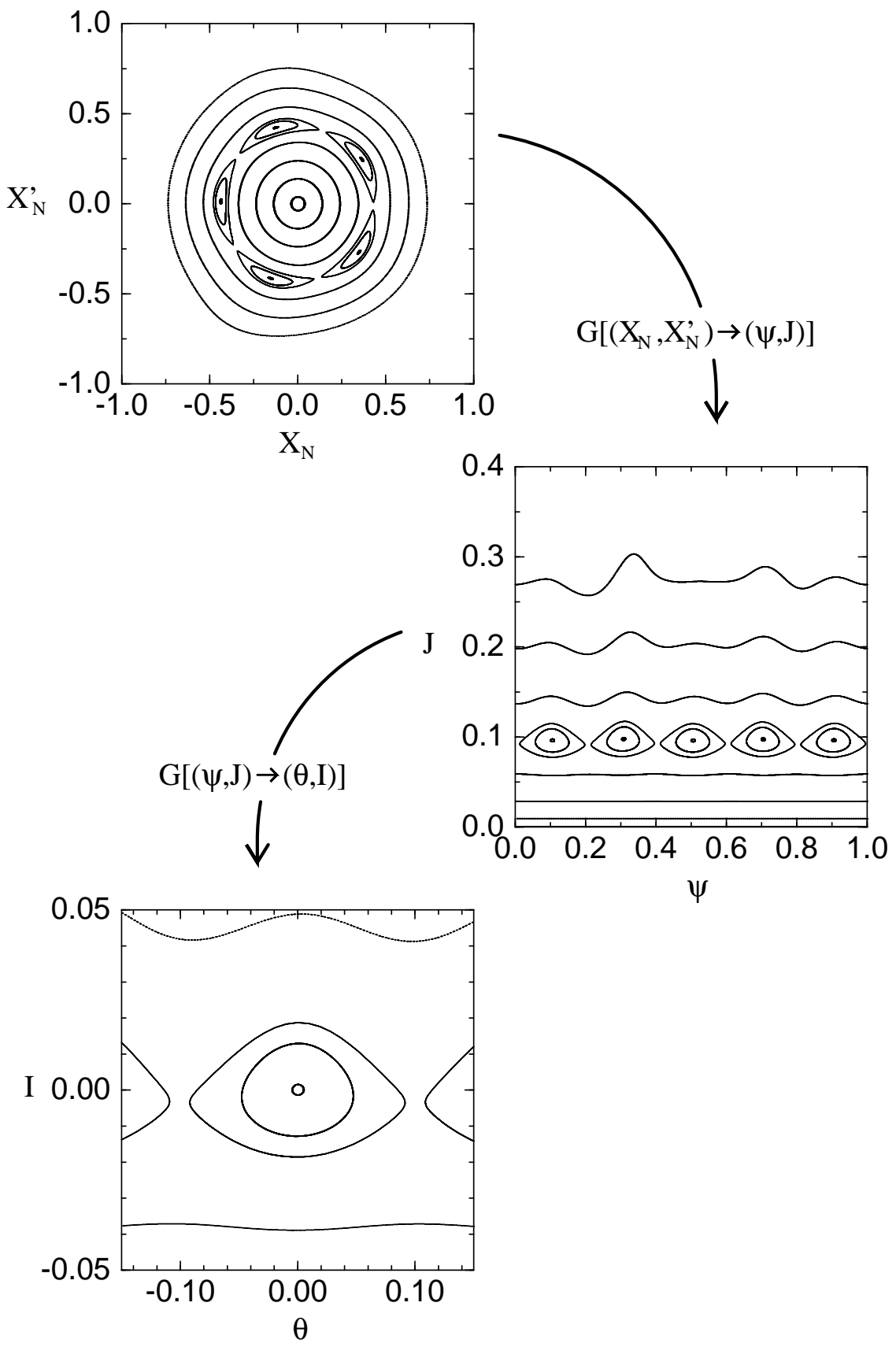

Figure 3.1: Isolated $5 Q_{x}$ resonance islands in various phase space coordinate systems. Data is taken from simulation and tracking using the octupole/decapole lattice described in Section 3.4, which drives the resonance seen here to first order in decapole strength. 
Hamiltonians.

Consider a synchrotron with a single thin nonlinearity of normalized strength $\tilde{b}_{n}$, where $n$ represents the order of the nonlinearity. Transverse single-particle motion around the ring consists of three parts: a linear phase advance to the nonlinearity, the nonlinear kick and another linear phase advance back to the starting point. Since the linear portion of this motion is already well-described by the Hamiltonian of the previous chapter, it is removed here by a reverse rotation through the ring while ignoring the nonlinearity. This has the effect of projecting the kick at the phase advance of the nonlinearity to a nonlinear one-turn map of the initial particle coordinates.

Denote the horizontal and vertical phase advances at the location of the nonlinearity by $\left(\phi_{n l, x}, \phi_{n l, y}\right)$ (as measured from a reference point) and the linear beta functions by $\left(\beta_{n l, x}, \beta_{n l, y}\right)$. The nonlinear kicks at this location in normalized coordinates are then given by substitution the of normalized coordinate definitions into the nonlinear kick expansion, Equation (2.6):

$$
\begin{aligned}
& \Delta x_{N}^{\prime}=-\tilde{b}_{n} \sum_{k=0}^{n / 2}\left(\begin{array}{c}
n \\
2 k
\end{array}\right)(-1)^{k} \beta_{n l, x}^{\frac{n+1}{2}-k} x_{N}^{n-2 k} \beta_{n l, y}^{k} y_{N}^{2 k} \\
& \Delta y_{N}^{\prime}=\tilde{b}_{n} \sum_{k=0}^{n / 2-1}\left(\begin{array}{c}
n \\
2 k+1
\end{array}\right)(-1)^{k} \beta_{n l, x}^{\frac{n-1}{2}-k} x_{N}^{n-2 k-1} \beta_{n l, y}^{k+1} y_{N}^{2 k+1} .
\end{aligned}
$$

This nonlinearity is a perturbation at a particular phase advance within the machine. To quantify this consider the "projection mapping":

$$
\left(\begin{array}{c}
\Delta x_{N} \\
\Delta x_{N}^{\prime} \\
\Delta y_{N} \\
\Delta y_{N}^{\prime}
\end{array}\right)=\mathbf{R}^{-1}\left(\phi_{n l, x}, \phi_{n l, y}\right)\left(\begin{array}{l}
\mathbf{K}_{x} \\
\mathbf{K}_{y}
\end{array}\right) \mathbf{R}\left(\phi_{n l, x}, \phi_{n l, y}\right)\left(\begin{array}{c}
x_{N} \\
x_{N}^{\prime} \\
y_{N} \\
y_{N}^{\prime}
\end{array}\right)
$$

where $\mathbf{R}$ is the $4 \times 4$ block diagonal linear rotation matrix

$$
\mathbf{R}\left(\phi_{x}, \phi_{y}\right) \equiv\left(\begin{array}{cccc}
\cos \phi_{x} & \sin \phi_{x} & 0 & 0 \\
-\sin \phi_{x} & \cos \phi_{x} & 0 & 0 \\
0 & 0 & \cos \phi_{y} & \sin \phi_{y} \\
0 & 0 & -\sin \phi_{y} & \cos \phi_{y}
\end{array}\right)
$$




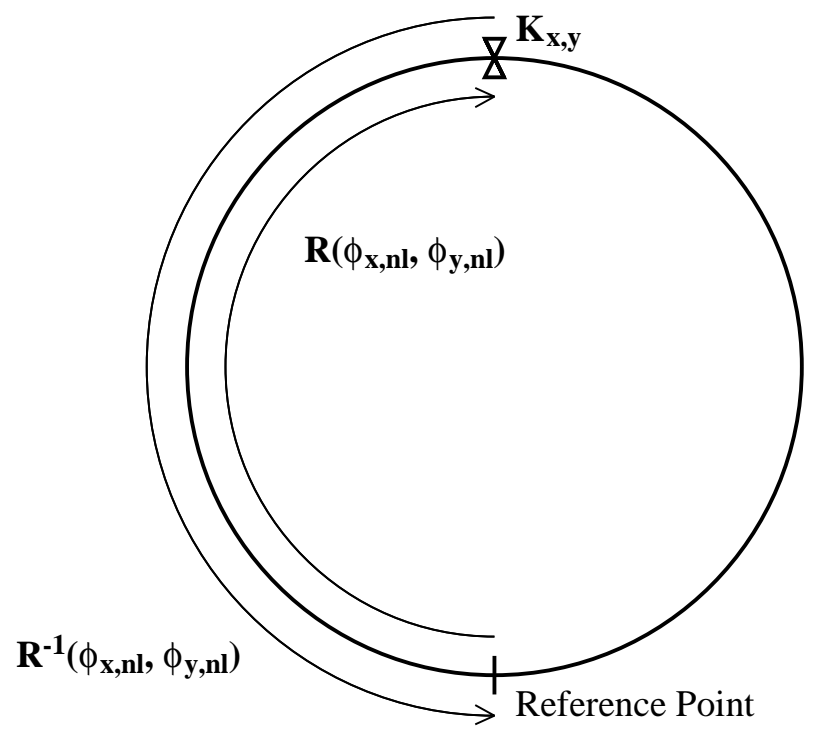

Figure 3.2: A simple lattice for the projection of a nonlinear kick onto a mapping of initial particle coordinates.

and the $\mathbf{K}_{i}$ symbolize the nonlinear kicks (3.1) in each plane. This accurately accounts for the nonlinear motion - the linear portion of the motion has been removed by the inverse rotation $\mathbf{R}^{-1}$.

If $x_{N}^{\prime}$ is the canonical momentum for $x$, the mapping of Equation (3.2) is actually integrable. A discrete one-turn nonlinear Hamiltonian $H_{1, n l}\left(x_{N}, x_{N}^{\prime}, y_{N}, y_{N}^{\prime}\right)$ can be written such that discrete forms of Hamilton's equations are obeyed as in the previous chapter:

$$
\left(\begin{array}{c}
\Delta x_{N} \\
\Delta x_{N}^{\prime} \\
\Delta y_{N} \\
\Delta y_{N}^{\prime}
\end{array}\right)=\left(\begin{array}{c}
\partial H_{1, n l} / \partial x_{N}^{\prime} \\
-\partial H_{1, n l} / \partial x_{N} \\
\partial H_{1, n l} / \partial y_{N}^{\prime} \\
-\partial H_{1, n l} / \partial y_{N}
\end{array}\right)
$$

The Hamiltonian can be written in these coordinates but it is much more meaningful to write it in action-angle coordinates $\left(\psi_{x}, J_{x}, \psi_{y}, J_{y}\right)$, which are related to 
the normalized coordinates via the canonical transformation

$$
\begin{aligned}
G_{1}\left(\left[x_{N}, x_{N}^{\prime}\right]\right. & \rightarrow[\psi, J])=-\frac{x_{N}^{2}}{2} \tan \psi \\
x_{N} & =\sqrt{2 J} \cos \psi \\
x_{N}^{\prime} & =-\sqrt{2 J} \sin \psi
\end{aligned}
$$

in each plane. Including the linear portion of the motion and denoting the unperturbed linear base tunes by $\left(Q_{x 0}, Q_{y 0}\right)$, we find the explicit form of the one-turn first-order nonlinear Hamiltonian:

$$
\begin{aligned}
H_{1}\left(\psi_{x}, J_{x}, \psi_{y}, J_{y}\right)= & 2 \pi Q_{x 0} J_{x}+2 \pi Q_{y 0} J_{y} \\
+\tilde{b}_{n} \sum_{k=0}^{n / 2} & \frac{n !(-1)^{k} 2^{\frac{n+1}{2}}}{(2 k) !(n-2 k+1) !}\left(\beta_{n l, x} J_{x}\right)^{\frac{n-2 k+1}{2}}\left(\beta_{n l, y} J_{y}\right)^{k} \\
& \times \cos ^{n-2 k+1}\left(\psi_{x}+\phi_{n l, x}\right) \cos ^{2 k}\left(\psi_{y}+\phi_{n l, y}\right),
\end{aligned}
$$

where the $n / 2$ limit in the sum is rounded up. This Hamiltonian may be written in another form as a useful ansatz:

$$
\begin{aligned}
H_{1}\left(\psi_{x}, J_{x}, \psi_{y}, J_{y}\right)= & 2 \pi Q_{x 0} J_{x}+2 \pi Q_{y 0} J_{y} \\
& +\frac{1}{2} \alpha_{x x} J_{x}^{2}+\alpha_{x y} J_{x} J_{y}+\frac{1}{2} \alpha_{y y} J_{y}^{2} \\
& +\sum_{k, l} V_{k l}\left(J_{x}, J_{y}\right) \cos \left(k \psi_{x}+l \psi_{y}+\phi_{k l}\right)
\end{aligned}
$$

The first line of the Hamiltonian contains the linear phase advance terms as in the previous chapter. The second line contains some of the so-called "shear terms", which are not phase dependent but instead cause action-dependent tunes via

$$
\begin{aligned}
& Q_{x}\left(J_{x}, J_{y}\right)=\left\langle\frac{\partial H_{1}}{\partial J_{x}}\right\rangle=Q_{x 0}+\frac{1}{2 \pi}\left(\alpha_{x x} J_{x}+\alpha_{x y} J_{y}\right) \\
& Q_{y}\left(J_{x}, J_{y}\right)=\left\langle\frac{\partial H_{1}}{\partial J_{y}}\right\rangle=Q_{y 0}+\frac{1}{2 \pi}\left(\alpha_{x y} J_{x}+\alpha_{y y} J_{y}\right)
\end{aligned}
$$

Here the brackets mean to average over all phases $\phi_{x}$ and $\phi_{y}$ - this phase averaging is explicitly incorrect in the case of resonant motion where only a certain subset of particle phases are reached. 
The last line of the Hamiltonian (3.7) contains phase dependent terms, or resonances, that are driven by this multipole, obtained by expanding the trigonometric functions in Equation (3.6). Equation (3.7) is a general form of the Hamiltonian for all orders of the nonlinear strength, with first order terms given exactly by Equation (3.6); these are tabulated for sextupole, octupole and decapole magnets in Table (3.1). The $V_{k l}$ coefficients are resonance strengths which depend on the actions, and the $\phi_{k l}$ are the relative phases of the resonance driving terms.

The treatment of several multipoles to first order is straightforward, because to that order there are no interactions between the nonlinearities. Thus the firstorder nonlinear Hamiltonian for any collection of multipoles is simply the sum of their individual nonlinear Hamiltonians. For higher order nonlinear contributions to the $\alpha_{i j}$ and $V_{k l}$ coefficients this procedure is inadequate, and other techniques must be used to evaluate these coefficients such as successive iteration of the discrete mapping (Peggs and Talman 1986), Deprit's algorithm (Michelotti 1986a,Michelotti 1986b) or application of successive Moser transformations (Merminga and $\mathrm{Ng} 1989$ ).

Examination of Table (3.1) demonstrates that sextupoles contain no shear terms to first order, but instead drive three sets of nonlinear resonances: $Q_{x}, 3 Q_{x}$, and $Q_{x} \pm 2 Q_{y}$. Shear terms quadratic in action, which correspond to detuning, are driven to second order in the sextupole strength; explicit formulae for this tune shift exist (Peggs and Talman 1986, Michelotti 1986a) and have been verified against simple particle tracking. Generally, the Hamiltonian of Equation (3.6) demonstrates that for a multipole kick of order $n$, the highest order resonance driven to first order in the multipole strength is $(n+1) Q=l$. For a head-on nonlinear beam-beam kick (see Equation (7.4)), in contrast, all even resonances are driven to first order in the small quantity parameterizing the kick.

The $5 Q_{x}$ resonance investigated in E778 is explicitly directly driven to third 


\begin{tabular}{|c|c|}
\hline $\begin{array}{c}\text { Hamiltonian } \\
\text { Coefficient }\end{array}$ & Lattice Value \\
\hline \hline$V_{1,0}$ & $\frac{\sqrt{2}}{4} \tilde{b}_{2} J_{x}^{1 / 2}\left[2 J_{x}-J_{y}\right]$ \\
\hline$V_{3,0}$ & $\frac{\sqrt{2}}{6} \tilde{b}_{2} J_{x}^{3 / 2}$ \\
\hline$V_{1, \pm 2}$ & $-\frac{\sqrt{2}}{8} \tilde{b}_{2} J_{x}^{1 / 2} J_{y}$ \\
\hline \hline$\alpha_{x x}$ & $\frac{3}{4} \tilde{b}_{3}$ \\
\hline$\alpha_{y y}$ & $\frac{3}{4} \tilde{b}_{3}$ \\
\hline$\alpha_{x y}$ & $-\frac{3}{2} \tilde{b}_{3}$ \\
\hline$V_{2,0}$ & $\frac{1}{2} \tilde{b}_{3} J_{x}\left[J_{x}-3 J_{y}\right]$ \\
\hline$V_{0,2}$ & $\frac{1}{2} \tilde{b}_{3} J_{y}\left[J_{y}-3 J_{x}\right]$ \\
\hline$V_{2, \pm 2}$ & $-\frac{3}{4} \tilde{b}_{3} J_{x} J_{y}$ \\
\hline$V_{4,0}$ & $\frac{1}{8} \tilde{b}_{3} J_{x}^{2}$ \\
\hline$V_{0,4}$ & $\frac{1}{8} \tilde{b}_{3} J_{y}^{2}$ \\
\hline$V_{5,0}$ & $\frac{\sqrt{2}}{20} \tilde{b}_{4} J_{x}^{5 / 2}$ \\
\hline$V_{3,0}$ & $\frac{\sqrt{2}}{4} \tilde{b}_{4} J_{x}^{3 / 2}\left[J_{x}-4 J_{y}\right]$ \\
\hline$V_{1,0}$ & $\frac{\sqrt{2}}{2} \tilde{b}_{4} J_{x}^{1 / 2}\left[J_{x}^{2}-6 J_{x} J_{y}+3 J_{y}^{2}\right]$ \\
\hline$V_{1, \pm 2}$ & $\frac{\sqrt{2}}{2} \tilde{b}_{4} J_{x}^{1 / 2} J_{y}\left[2 J_{y}-3 J_{x}\right]$ \\
\hline$V_{3, \pm 2}$ & $-\frac{\sqrt{2}}{2} \tilde{b}_{4} J_{x}^{3 / 2} J_{y}$ \\
\hline$V_{1, \pm 4}$ & $\frac{\sqrt{2}}{4} \tilde{b}_{4} J_{x}^{1 / 2} J_{y}^{2}$ \\
\hline & \\
\hline & \\
\hline & \\
\hline &
\end{tabular}

Table 3.1: First-order Hamiltonian coefficients in Equation (3.7) for sextupole, octupole and decapole magnets. The beta functions scale into these with the actions $J_{x, y}$, but have been set equal to one here for clarity. For the octupole-decapole lattice octupole detuning coefficients $\alpha_{i j}$ are tripled due to the presence of three octupoles. 
order in sextupole strength (Merminga and $\mathrm{Ng}$ 1992,Michelotti 1986b). However, it has been hypothesized that the structure of this resonance may be adequately reproduced at a lower order by interference between the $3 Q_{x}$ and $2 Q_{x}$ resonances (Michelotti 1991). This issue remains a subject of debate; in either event the resonance strength driving by sextupoles is smaller than the first-order approach presented here.

\subsection{THE ONE-DIMENSIONAL NONLINEAR N-TURN HAMILTONIAN}

The general multi-resonance Hamiltonian as given in Equation (3.7) is extremely difficult to investigate analytically. Instead we examine an explicitly one-dimensional case $\left(J_{y}=0\right)$, dropping the $(x, y)$ subscripts. We also assume that the horizontal base tune $Q_{0}$ is very close to a one-dimensional resonance: $Q_{0} \equiv M / N+$ $\delta_{Q}$, where $\delta_{Q} \ll 1 / N$. Under these conditions, we shall show that the Hamiltonian is approximately that of a isolated resonance model with a family of elliptically stable N-turn fixed points and that motion around any of these stable fixed points can be transformed to a form approximating that of a classical free pendulum (Chirikov 1979).

One difficulty of the discrete Hamiltonian is the discontinuous nature of the phase advance - if the tune is not near an integer the net phase advance every turn is large. However, with the assumption that the tune is near the resonance $M / N$, the accumulated phase advance over $N$ turns is very close to $2 \pi M$ and thus effectively small. It therefore makes sense to look at a difference Hamiltonian that reproduces a Poincaré surface of section not every turn, but every $N$ turns. Alternatively, one could embed this system in a system of higher dimensionality (Vivaldi 1984), but the method of integrating (summing) the Hamiltonian explicitly at low orders is more intuitive for this application.

When examining N-turn motion, the time variable needs to be changed from 
$t=0,1, \ldots$ to "N-turns", $T \equiv t / N=0,1, \ldots$, as stated in the previous chapter. One must also be careful to distinguish the relevant timescales for frequencydomain variables; from here on any frequency variable, say $Q$, is defined to be the frequency of oscillations in the one-turn timescale:

$$
\sin (2 \pi Q t)=\sin (2 \pi Q N T)
$$

Dropping $x$ and $y$ subscripts and setting $J_{y}=0$, the general one-turn onedimensional Hamiltonian can be written from Equations (3.6) and (3.7) as

$$
H_{1}(\psi, J)=2 \pi Q_{0} J+\frac{1}{2} \alpha J^{2}+\sum_{k, k^{\prime}} V_{k k^{\prime}} J^{k / 2} \cos \left(k^{\prime} \psi+\phi_{k k^{\prime}}\right)
$$

It is important to note that the action dependence has been removed from the resonance strength $V_{k k^{\prime}}$ here and inserted as a sum of its own. Summing this Hamiltonian over $N$ turns and approximating the action $J$ as constant over these $N$ turns gives

$$
\begin{aligned}
H_{N}(\psi, J)= & 2 \pi N \delta_{Q} J+\frac{N}{2} \alpha J^{2} \\
& +\sum_{k, k^{\prime}} V_{k k^{\prime}} J^{k / 2} \sum_{i=0}^{N-1} \cos \left[k^{\prime}\left(\psi+\frac{2 \pi i M}{N}+2 \pi i \delta_{Q}\right)+\phi_{k k^{\prime}}\right] .
\end{aligned}
$$

Only the linear contribution to the phase advance has been included in the resonance terms since this analysis is to first order in the nonlinear terms $V_{k k^{\prime}}$ and $\alpha$. The sum over $i$ in (3.11) is easily found by using the identity

$$
\begin{aligned}
\sum_{i=0}^{N-1} \cos (A+i B) & =\mathcal{D}(B, N) \cos \left(A+\frac{N-1}{2} B\right), \\
\mathcal{D}(B, N) & \equiv \frac{\sin (N B / 2)}{\sin (B / 2)} .
\end{aligned}
$$

$\mathcal{D}(B, N)$ is extremely similar to the Dirichlet kernel found in the theory of Fourier series; it is very strongly peaked at values of $B$ where the difference between $B / \pi$ and the nearest integer is much less than $1 / N$, in which case its value is 
approximately $N$. In this case $B / \pi=2 k^{\prime}\left(M / N+\delta_{Q}\right)$, and so the only terms that contribute significantly are terms where $k^{\prime}$ is a multiple of $N$. (This is in practice an approximation, because very large resonance strengths, possibly from lower order terms, can overcome this suppression.) For typical multipole magnets encountered in accelerators (i.e. small order, $n$ ) the $k^{\prime}=N$ term will dominate, and there will be only one resonant term that contributes. In this case we have the isolated resonance N-turn Hamiltonian

$$
\begin{aligned}
H_{N}(\psi, J)= & 2 \pi N \delta_{Q} J+\frac{N}{2} \alpha J^{2} \\
& +N V_{N} J^{N / 2} \cos \left[N \psi+\phi_{N}\right] \\
& + \text { suppressed resonance terms }
\end{aligned}
$$

where a constant phase offset $N(N-1) \pi \delta_{Q}$ has been absorbed into $\phi_{N}$.

\subsection{PARAMETERIZATION AND CHARACTER OF RESONANT MOTION}

Now that we have this Hamiltonian, we can answer the question of how the small nonlinearity distorts the normal linear phase space motion of particles within an accelerator. With no resonance driving at all (i.e. only linear and shear terms)

particles still trace out circular trajectories in $\left(x_{N}, x_{N}^{\prime}\right)$ space, with radii $\sqrt{2 J}$ and phases $\psi$. However, the particle tunes are action-dependent due to the shear terms in the Hamiltonian. This section will show that when a resonance term is introduced with shear terms present, structures called "resonance islands" are formed in phase space that can be parameterized in a way completely equivalent to free pendulum motion.

Applying the discrete forms of Hamilton's equations to the N-turn Hamiltonian of Equation (3.13) gives, over $N$ turns,

$$
\Delta \psi=\frac{\partial H_{N}}{\partial J}=2 \pi N \delta_{Q}+N \alpha J+\frac{N^{2}}{2} V_{N} J^{N-2 / 2} \cos (N \psi)
$$


and

$$
\Delta J=-\frac{\partial H_{N}}{\partial \psi}=N^{2} V_{N} J^{N / 2} \sin (N \psi)
$$

The map described by Equations (3.14) and (3.15) exhibits N-turn fixed points when both $\Delta J$ and $\Delta \psi$ are zero. The constraint $\Delta J=0$ gives one trivial solution $\left(J_{f p}=0\right)$ and $2 N$ fixed points at the phases $\psi_{f p}=k_{r e s} \pi / N$, where $k_{r e s}$ is an integer. Using these phases in Equation (3.14) then gives the equation for $J_{f p}$ :

$$
2 \pi \delta_{Q}+\alpha J_{f p} \pm \frac{N}{2} V_{N} J_{f p}^{\frac{N-2}{2}}=0
$$

where the top sign refers to even $k_{r e s}$, and the bottom to odd $k_{r e s}$. This equation for the fixed-point action is in general transcendental, but can be exactly solved for all $N$ below 7 . Note that when no positive real solutions exist for $J_{f p}$, there are no fixed points. In practice the detuning term is usually much larger than the resonance term, in which case an approximate solution is $J_{f p} \approx-2 \pi \delta_{Q} / \alpha$.

We can now apply a last linear transformation to the Hamiltonian, by expanding the N-turn Hamiltonian (3.13) around these fixed points with the linear (canonical) translation

$$
\begin{aligned}
& \psi=\psi_{f p}+\theta, \\
& J=J_{f p}+I .
\end{aligned}
$$

The expansion action $I$ is assumed to be small with respect to the magnitudes of the fixed points action, so $I \ll J_{f p}$; no such constraint is placed on $\theta$. The final Hamiltonian is then found to be

$$
H_{N}(\theta, I)=\frac{N}{2} \alpha I^{2} \pm N V_{N} J_{f p}^{N / 2} \cos (N \theta)+\mathcal{O}\left(V_{N} I\right)
$$

If $\alpha V_{N}$ is negative, there is a family of $N$ elliptic (stable) fixed points (at phases $\theta=0,2 \pi / N, \ldots$, corresponding to even $\left.k_{r e s}\right)$, surrounded by a separatrix intersecting another family of $N$ hyperbolic fixed points (at phases $\theta=\pi / N, 3 \pi / N, \ldots$, corresponding to odd $k_{r e s}$ ); if $\alpha V_{N}$ is positive the converse is true. We assume the 
latter and choose the negative sign in this Hamiltonian to examine local motion around a stable fixed point.

Motion within the separatrix is termed "resonant" or "phase-locked" — particles within this region have a long-term average phase advance per turn, or tune, of exactly $M / N$. Particle trajectories just outside of the separatrix are distorted but nonresonant, as seen in Figure (3.1). Consider a set of particles populating one of these resonance islands at one particular time in the accelerator; this ensemble of particles will advance from island to island turn by turn in a completely coherent fashion. A position measurement of the centroid of this distribution will exhibit a coherent oscillation. This oscillation can in practice be observed on a online spectrum analyzer as a "persistent signal" at exactly the resonant tune, and with unperturbed resonance islands it typically has an exceptionally long lifetime of millions of turns.

Ignoring terms of order $V_{N} I$ and higher, Equation (3.18) is a free pendulum Hamiltonian and the equation of motion for $\theta$ that arises is

$$
\begin{aligned}
\ddot{\theta} & =-N^{3} \alpha V_{N} J_{f p}^{N / 2} \sin (N \theta) \\
& =-N\left(2 \pi Q_{\mathrm{I}}\right)^{2} \sin (N \theta)
\end{aligned}
$$

where the time derivatives are taken with respect to the "N-turn" time variable $T$, and $Q_{\mathrm{I}}$ (the "island tune", or frequency of small librations of this pendulum system) is defined by

$$
Q_{\mathrm{I}} \equiv \frac{1}{2 \pi} N J_{f p}^{N / 4} \sqrt{\left|\alpha V_{N}\right|}
$$

A typical island tune observed in the E778 experiment is approximately 0.006 , with similar values given by tracking with realistic nonlinear magnet strengths.

The island half-width $\delta I$ can also be found from the Hamiltonian, because this Hamiltonian is autonomous. The separatrix is a contour along which the Hamiltonian is constant, and so the Hamiltonian value at an unstable fixed point 
is equal to the Hamiltonian value at a stable fixed point phase with actions $\pm \delta I$ :

$$
\begin{aligned}
H_{N}(\theta=\pi / N, I=0) & =H_{N}(\theta=0, I= \pm \delta I) \rightarrow \\
\delta I & =2 J_{f p}^{N / 4} \sqrt{\left|\frac{V_{N}}{\alpha}\right|} .
\end{aligned}
$$

Knowledge of the base tune $Q_{x 0}$ (and thus $\delta_{Q}$ ), the island tune $Q_{\mathrm{I}}$ and the detuning $\alpha$ allows deduction of the fixed point action $J_{f p}$, island size $\delta I$ and resonance driving strength $V_{N}$.

Since the motion is that of a pendulum within the resonance island, particles at different actions within the island circulate with different frequencies or tunes. These tunes range from the island tune $Q_{\mathrm{I}}$ at actions near zero to zero frequency on the separatrix, and can be written in terms of elliptic integrals. This nonlinear dependence of frequency with action (or amplitude) of the pendulum system makes measurement of $Q_{\mathrm{I}}$ more difficult, for only particles with actions near the fixed point action actually rotate in the island with this tune.

\subsection{FIRST ORDER NONLINEAR TRACKING AND SIMULATION}

For the issues investigated in this thesis, it was deemed necessary to design a dedicated simulation program that tracked a minimal lattice which drives relevant terms in the nonlinear Hamiltonian to first order. The Octupole-Decapole model lattice is designed with this goal in mind; it also uses sets of specially placed quadrupoles to independently control tune modulation and beta modulation at the resonance-driving nonlinearity. For simplicity, all beta functions are set at one meter in the following discussion and within the tracking codes themselves.

The lowest order multipoles that drive action-dependent nonlinear detuning of the form $\alpha J^{2}$ are octupoles, as seen in Table (3.1). To first order all resonance terms contributed by the octupoles should vanish in the simulation. This enables complete separation between detuning, the function of the octupoles, and resonance driving from the decapole. It is possible to eliminate all but one of the first 


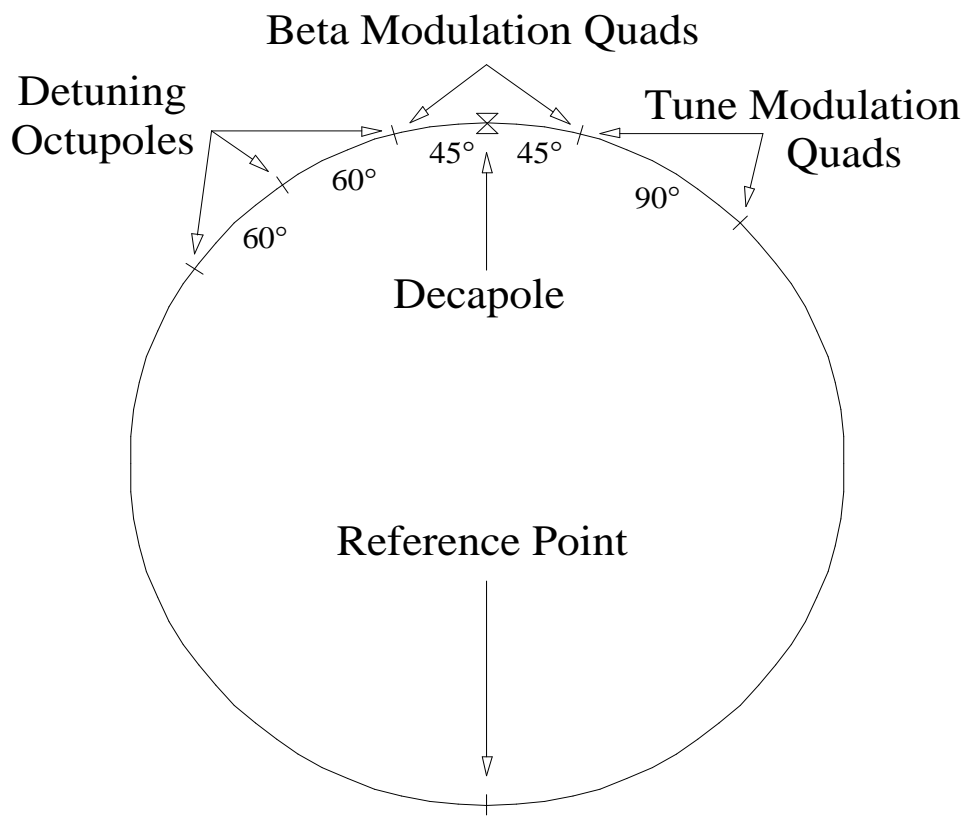

Figure 3.3: The octupole-decapole lattice.

order octupole resonances, the diagonal coupling resonance $2 Q_{x}-2 Q_{y}$, by driving three octupoles with the same strength, spaced apart by a betatron phase advance of $60^{\circ}=2 \pi / 3$ between each octupole. This also triples the phase-independent detuning coefficients $\alpha_{i j}$ over their single octupole values. The decapole is used to drive the $5 Q_{x}$ resonance, motivated by the study of this resonance in E778.

To investigate the effects of tune modulation and beta modulation, the linear phase advances in the lattice were set to constant values and only special quadrupoles explicitly introduced into the lattice were modulated. Three quadrupoles are sufficient to independently control the tune modulation of the lattice and beta modulation at the resonance-driving decapole. The tune shift from a single small quadrupole error is given by Equation (2.22), recalling $\beta_{x}=\beta_{y}=1 \mathrm{~m}$ for this lattice,

$$
\Delta Q(\text { quad })=\frac{\tilde{b}_{1}}{4 \pi}
$$


This is independent of the phase of the quadrupole within the machine, so a pair of modulated quadrupoles driven with the same sign will produce tune modulation with strength $q=2 \Delta Q=\tilde{b}_{1} / 2 \pi$. The beta modulation created by a quadrupole error is given by Equation (4.4),

$$
\Delta \beta(s)=\frac{\tilde{b}_{1}}{2}\left[\sin \left(2\left|\phi(s)-\phi_{q u a d}\right|\right)-\cos \left(2\left|\phi(s)-\phi_{q u a d}\right|\right) \tan \left(2 \pi Q_{0}\right)\right] .
$$

Two quadrupoles driven with equal strength, opposite sign and $90^{\circ}$ apart in phase advance will destructively interfere outside of this phase advance. They constructively interfere within the $90^{\circ}$ phase advance, and at a point exactly halfway in between them they will modulate the beta function with strength $b=\Delta \beta=\tilde{b}_{1}$, with no tune modulation since the quadrupoles are driven with equal and opposite strengths.

The elements of this lattice are shown in Figure (3.3); the phase advances outside the cluster of quadrupoles and nonlinear elements are set by the two constraints that the linear tunes are some set values, say $\left(Q_{x 0}, Q_{y 0}\right)$, and the decapole is positioned exactly halfway through the lattice from the reference point, so $\phi_{d e c}=\pi Q_{0}$ in each plane.

A one-dimensional tracking program, OdTrack, has been written which tracks single particles through horizontal phase space in this lattice and includes the effects of either or both tune modulation and beta modulation. The inputs to this program include the horizontal base tune, the octupole and decapole strengths $\tilde{b}_{3}$ and $\tilde{b}_{4}$, the amplitude and period (in turns) of the modulation and the number of turns to track. Particles can be launched at a variety of amplitudes but only at a single phase during every execution of the simulation. Output includes the phase space coordinates of every particle for each synchrotron period and the measured tune of each particle over the entire tracking run.

Timing tests on a Sparcstation IPX show an iteration CPU time of $33 \mu$ s per 
turn, making million-turn tracking with this lattice easily accessible. Raising the timescales by several orders of magnitude to examine thousands of millions of turns (or millions of synchrotron periods) of evolution still only requires tens of hours of processing time, and is quite feasible.

The prediction for the island tune of Equation (3.20) is proportional to the square root of both the detuning $\alpha$ and the resonance driving strength $V_{5}$. However, with the approximation that $V_{5} J_{f p}^{\frac{-1}{2}} \ll \alpha$ in Equation (3.16) for the fixed point action $J_{f p}$, it is apparent that the fixed point action also varies inversely with the detuning. Therefore, since the resonance strength $V_{N}$ is driven to first order in the decapole strength and the detuning is driven to first order in octupole strength (see Table [3.1]), the island tune is expected to vary as:

$$
Q_{\mathrm{I}} \propto \tilde{b}_{3}^{\frac{-3}{4}} \tilde{b}_{4}^{\frac{1}{2}}
$$

Figure (3.4) shows a comparison of the theoretical prediction for $Q_{\mathrm{I}}$ to tracking in this lattice while varying the octupole strength, showing the scaling of (3.24) for small $\tilde{b}_{3}$. As this strength becomes nonperturbatively large (on the order of $3 \tilde{b}_{3} \approx 1$, since there are three octupoles in this lattice), the naive first order predictions fail. This is due to a higher-order contribution to the resonance driving by the octupoles which cannot be ignored in this limit. However, for octupole strengths smaller than $.1 \mathrm{~m}^{-3 / 2}$, the results of first order perturbation theory appears adequate.

Figure (3.5) shows a similar comparison while varying the decapole strength, keeping the octupole strengths constant. Here the approximation begins to fail at lower orders as the $3 Q_{x}$ resonance, also driven to first order in $\tilde{b}_{4}$, begins to distort the phase space where the resonance islands are located. For decapole strengths less than $10^{-3} \mathrm{~m}^{-2}$, at the octupole strength listed, the prediction and scaling of (3.24) agree with tracking. 


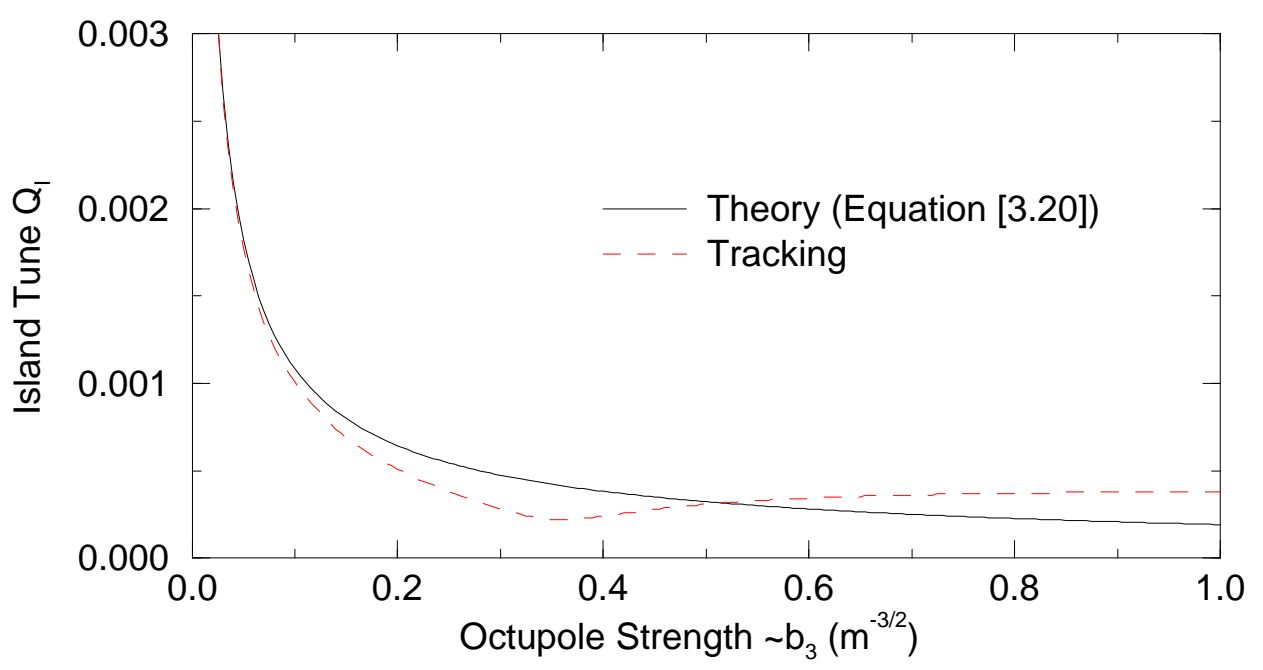

Figure 3.4: A comparison of the theoretical prediction for the island tune $Q_{\mathrm{I}}$ to tracking in the octupole-decapole lattice, varying the octupole strength $\tilde{b}_{3}$. This comparison is for the $5 Q_{x}$ resonance $\left(Q_{0}=2 / 5\right)$ with $\delta_{Q}=-0.02$, and the decapole strength is constant at $\tilde{b}_{4}=5 \cdot 10^{-4} \mathrm{~m}^{-2}$.

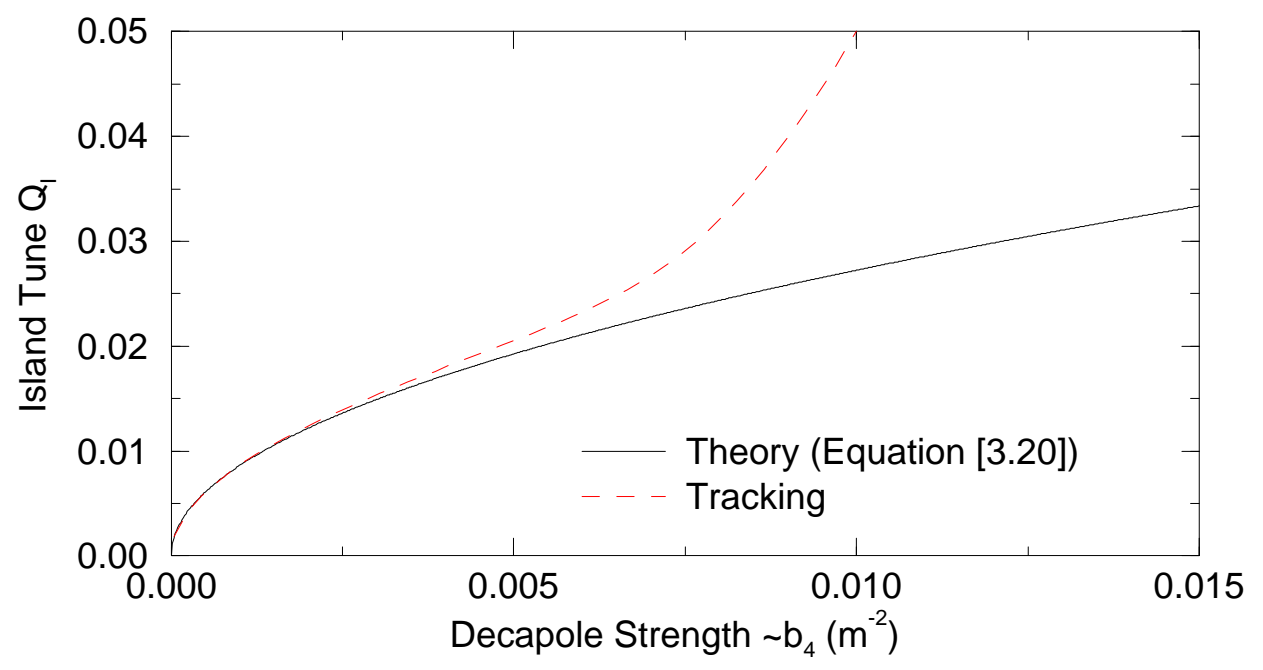

Figure 3.5: A similar comparison as the above figure, now keeping the octupole strength constant at $\tilde{b}_{3}=10^{-2} \mathrm{~m}^{-3 / 2}$ and varying the decapole strength $\tilde{b}_{4}$. 


\subsection{MEASUREMENTS IN REAL ACCELERATORS}

The detuning coefficient $\alpha$ can be experimentally measured in several ways. One method is to kick the beam transversely and observe the resulting tune shift at this betatron amplitude. This is typically only possible if the beam is small enough such that there is no appreciable tune spread over the beam distribution, due to the presence of tune spread decoherence as discussed in the next paragraph. This is also the method used by tracking programs to measure the tune dependence on amplitude, because the phase advance for a single particle can be measured to the computational precision and there is no tune spread or decoherence in single-particle motion.

With a moderately sized beam, the detuning coefficient can be measured by observing the decoherence time of a bunch kicked into a non-resonant section of phase space. Particles that comprise a kicked bunch will have different tunes (because of their different amplitudes after the kick), and so the distribution will "decohere", with the phases of particles with smaller tunes lagging further and further behind those of particles with larger tunes. The timescale for this phenomena to decohere the beam is

$$
\tau(\text { decohere })=\frac{1}{\left(\frac{d Q}{d J}\right)_{J(\text { kick })} \cdot \sigma},
$$

where $J$ (kick) is the action of the kick and $\sigma$ is the size of the beam measured in units of the action. For conditions experienced in experiment E778, the typical decoherence time is tens to hundreds of machine turns. This phenomenon has been previously studied, both within the context of E778 (Chao et. al. 1987b, Chao et. al. 1988, Merminga 1989) and in other environments (Lee et. al. 1991, Byrd 1992). It gives a quantitative measurement of the detuning strength even when the coherent oscillation decays rapidly and direct measurement of the tune at the new 
betatron amplitude is impossible. An illustration of this decoherence is shown in Figure (3.6), and an actual experimental turn-by-turn position measurement showing this decoherence is shown in Figure (3.9).

With this independent measure of $\alpha$, one now needs to know the island tune or island width in order to deduce the resonance strength. The island width has been measured in past E778 experimental runs by observing the fraction of kicked beam trapped within a resonance island (Chao et. al. 1988, Merminga 1989). A typical value of the physical island width, $\sqrt{\delta I \beta_{x}}$, reported by Merminga is half a millimeter. This method usually can only give general estimates of $\delta I$, and relies on fine calibration of the kicker.

Instead, recent experiments have concentrated on measuring $Q_{\mathrm{I}}$, the island tune, in machines where strong resonances have been driven by explicitly introduced nonlinearities. Sextupoles have predominantly been used, since they are the lowest order nonlinear multipoles and are commonly found in many accelerators to correct chromatic focusing errors. The procedure of finding and populating such resonance islands in the phase space of a real machine is greatly simplified by the appearance of "persistent signals" when a significant fraction of the beam is captured within a resonance island. When this occurs, the trapped beam exhibits a coherent oscillation with an exceptionally long lifetime - millions of turns and the frequency spectrum of the beam on an online spectrum analyzer shows a long-lived peak exactly at the resonant frequency.

One possible method to determine the island tune is to examine the coherent motion of a bunch kicked into a resonance island. If the bunch is small enough (ie. has an RMS size much smaller than the size of the island), it behaves essentially as a single particle for times much smaller than it's decoherence time within the island. The resulting coherent motion within the resonance allows direct measurement of $Q_{\mathrm{I}}$; such an approach was adopted by the CE22 collaboration at 
IUCF (Lee et. al. 1991). Figure (3.7) shows a representation of such a kick applied to the resonant system seen in Figure (3.6).

In the Fermilab Tevatron, beam sizes are typically large compared to the size of induced resonance islands; creation of larger resonance islands is impractical due to physical aperture considerations. This means that when the beam is kicked onto the resonance, the entire resonance island is populated. There is still a strong persistent signal corresponding to the coherent motion from island to island, but there is no local coherent motion of the particles trapped within the resonance island that allows direct observation of $Q_{\mathrm{I}}$. Figure (3.8) shows a symbolic representation of such a kick, including the decoherence of the portion of the beam not trapped within the resonance island. A sample turn-by-turn position measurement from E778 showing this decoherence and a persistent signal is seen in Figure (3.10), showing beam capture on a $5 Q_{x}$ resonance island chain.

There are various frequency-domain methods of examining the distribution of particles filling a single resonance island (Chen 1990). For example, if the resonance island is completely but not evenly populated, the discrete Fourier transform of a BPM measurement taken every $N$ turns should show a low-frequency element similar to that of an ensemble of driven pendula, dropping off quickly into background noise at frequencies near the island tune.

Since the distribution of particles within the resonance island was indeed expected to be relatively even due to the nature of the beam size used in E778, another method is investigated in this thesis. Resonantly captured beam was excited with a set of very weakly modulated quadrupoles, modulating the tune and effectively turning the ensemble of free pendula into driven pendula. The frequency response of the beam was then correlated to the frequency of the modulation for a reasonable measurement of the island tune. 

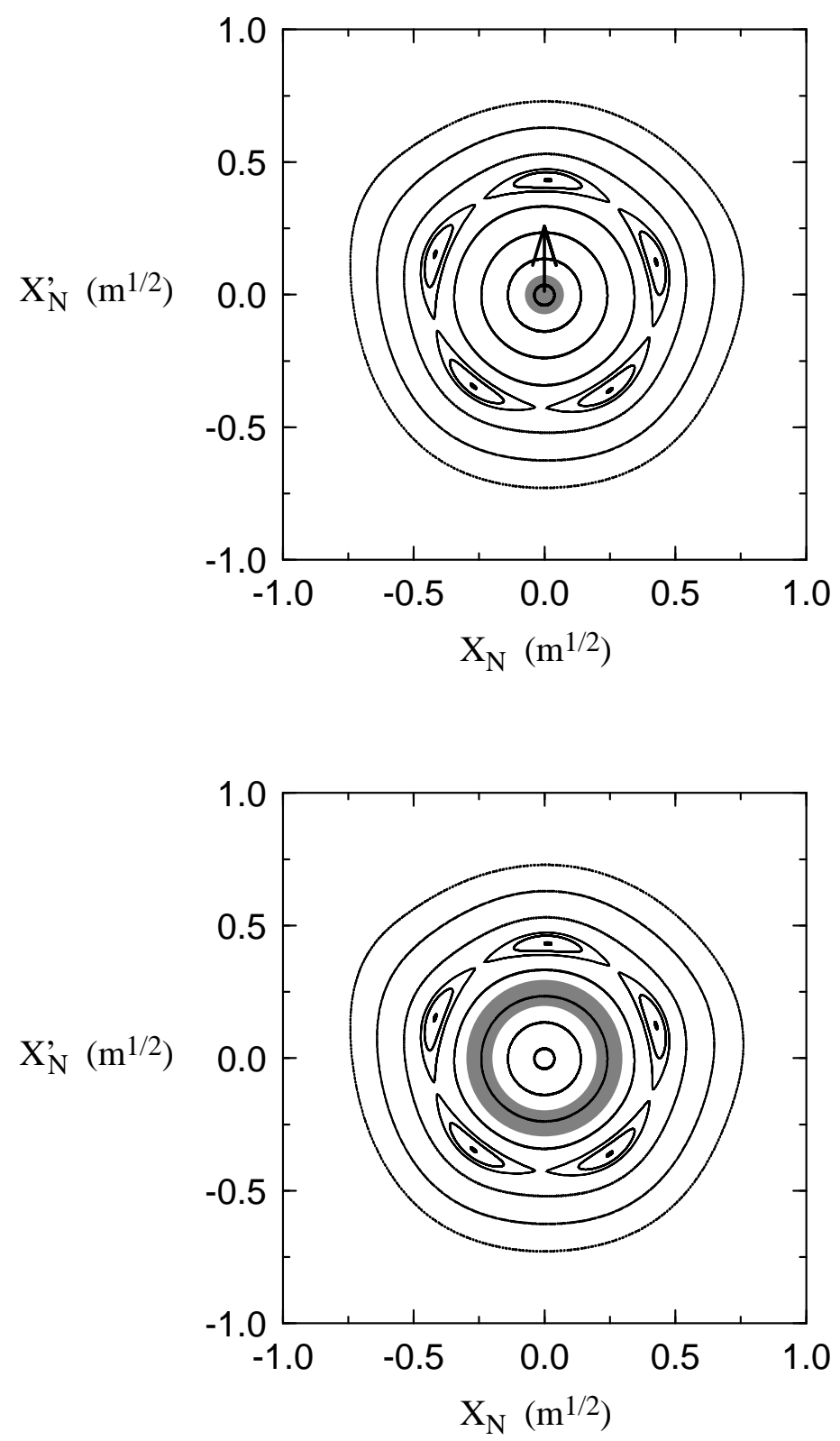

Figure 3.6: Beam decoherence from a kick into a sheared non-resonant section of phase space. The intermediate distribution has a centroid position that decays with time and approaches a final annular distribution (which has a larger emittance than the initial distribution and a centroid position of zero) over approximately hundreds of turns. 

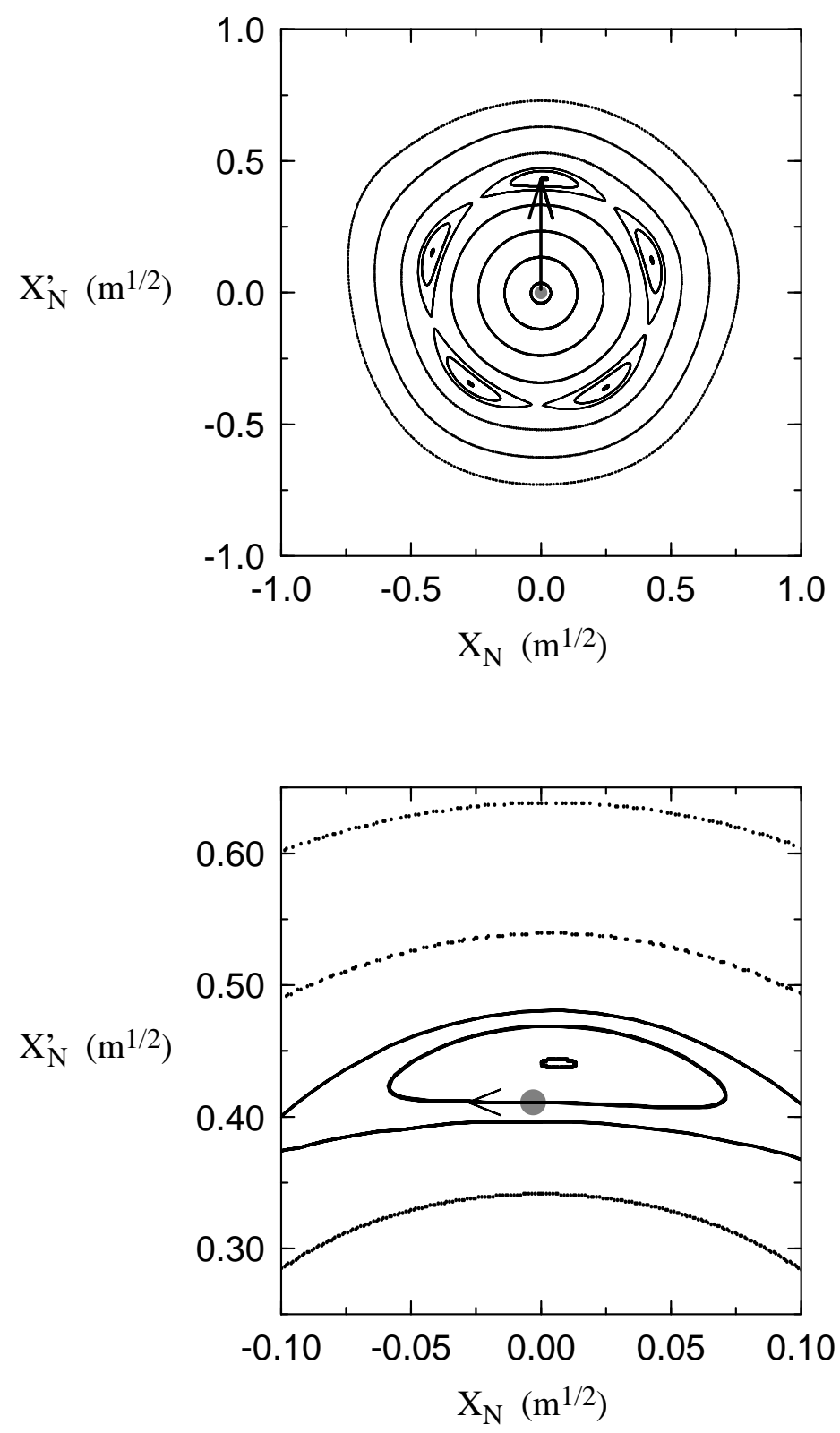

Figure 3.7: Phase space plot of a small beam kicked into an $N=5$ nonlinear resonance island. Measurement of the centroid of the kicked distribution every 5 machine turns shows coherent motion around the center of the island, making direct measurement of the island tune $Q_{\mathrm{I}}$ possible. 

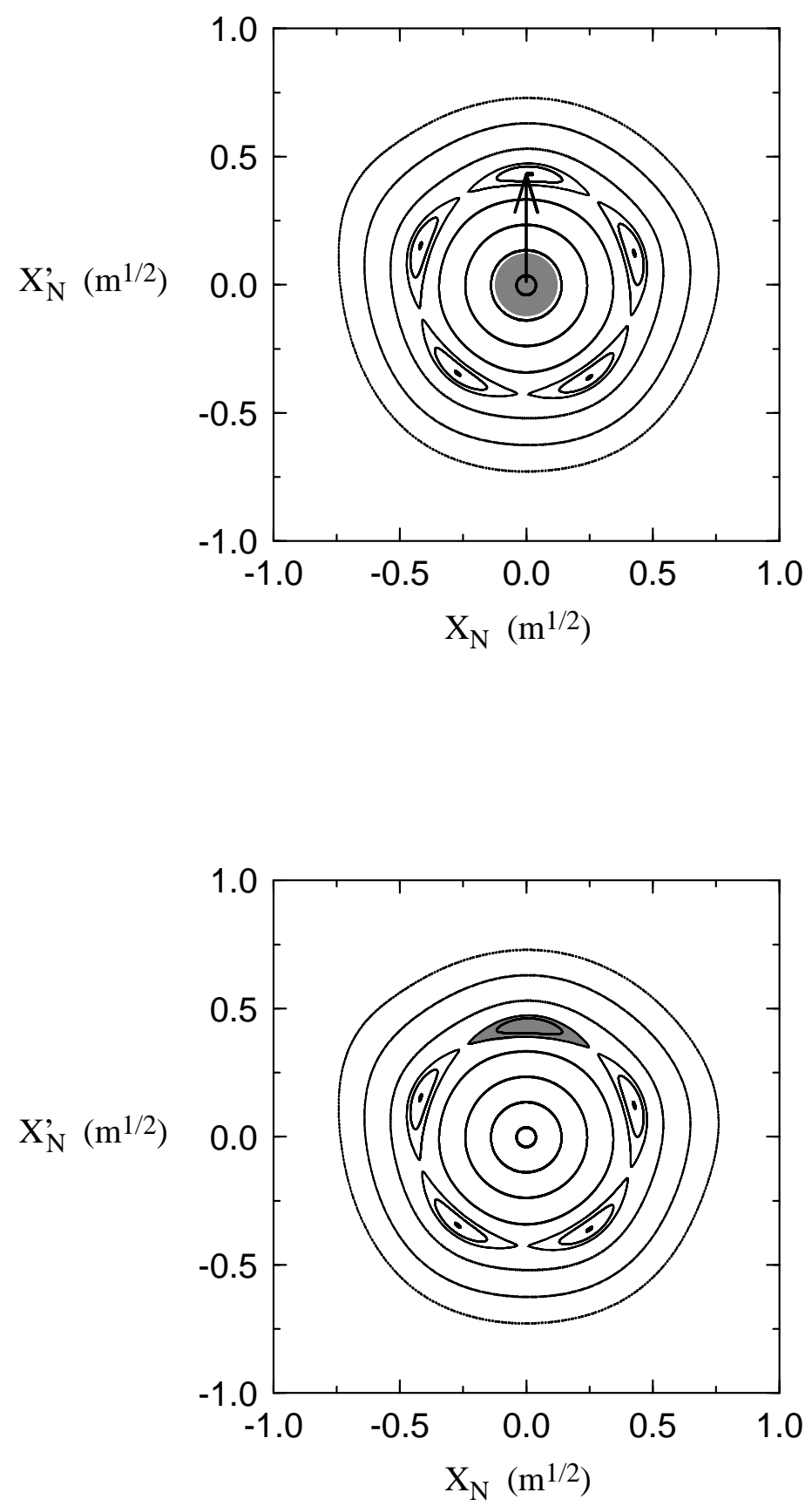

Figure 3.8: Phase space plot of a large beam kicked into an $N=5$ nonlinear resonance island. The untrapped portion of the beam decoheres and does not contribute to the coherent centroid motion. The trapped portion coherently moves between resonance islands, but shows no evidence of the island tune $Q_{\mathrm{I}}$. 


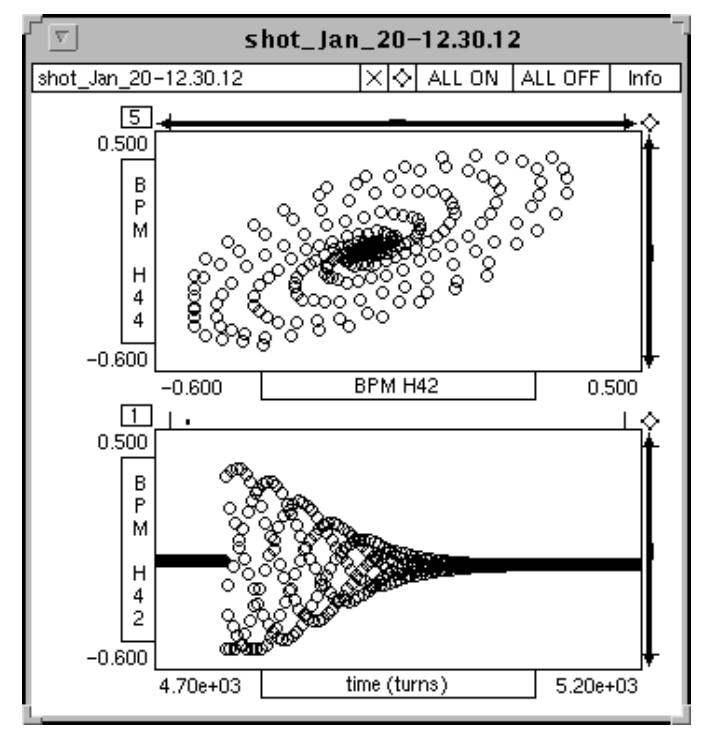

Figure 3.9: Sample E778 turn-by-turn data at kick time, showing the kick and gaussian decoherence. Kicker voltage is $11 \mathrm{kV}$, in the E778 91_0 lattice. The graphics are produced by the kaspar graphics program. The decoherence pictured has a timescale of approximately 100 turns.

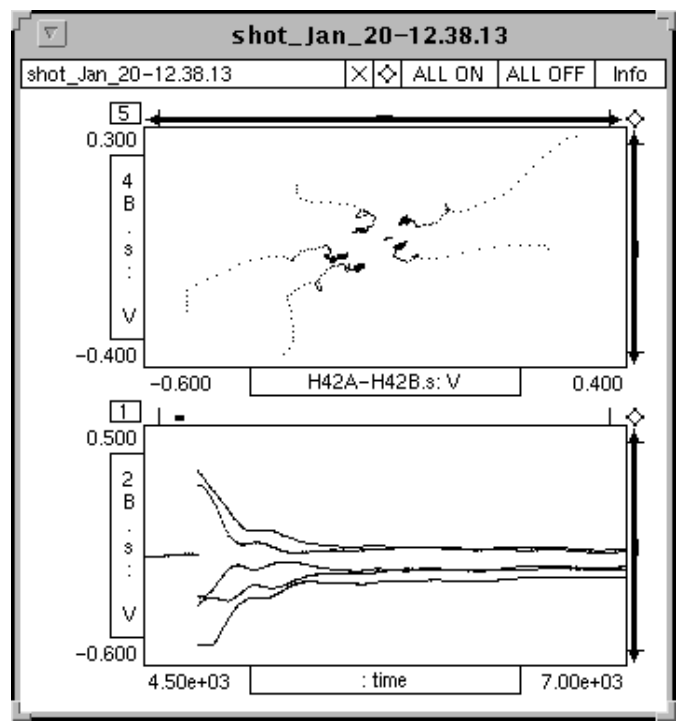

Figure 3.10: Sample turn-by-turn data at kick time for two BPMs separated by a betatron phase of approximately 70 degrees, showing the kick and production of a $Q_{x}=20.40$ persistent signal. Kicker voltage and lattice are as in above figure. Only 2500 turns are pictured here, but this coherent oscillation survives for millions of turns. 


\section{CHAPTER 4 \\ PERTURBATIONS OF NONLINEAR RESONANCES - TUNE MODULATION AND BETA MODULATION}

The effects of various types of perturbations on particle motion influenced by an isolated nonlinear resonance are investigated in this chapter. Two types of perturbation are of particular interest here: modulation of the machine tune and modulation of the linear beta functions at the nonlinear magnets driving the resonance.

Modulation effects are important for a variety of reasons. From a general dynamic viewpoint, the separatrices surrounding nonlinear resonance islands are extremely fragile under small perturbations such as modulations because they are homoclinic, or asymptotically joined to the unstable fixed points of the resonances (Lichtenberg and Lieberman 1983, Vivaldi 1984). As the modulation strength grows motion becomes stochastic in a widening area around the separatrix; this stochasticity can then act as a noise source for diffusion models that may be important mechanisms limiting the luminosity lifetimes of present and future colliders. One of these diffusion models, aptly named modulational diffusion, is described in greater detail in Chapter 7. Tune modulation is also important in study of the beam-beam interaction where its inclusion is necessary to reconcile operational observations with simulation and theory (Peggs and Talman 1986, Saritepe and Peggs 1991). Modulation methods have been proposed for use in crystal channeling and parasitic beam extraction at the SSC (Gabella et. al. 1992) where controlled RF modulation would be used to create trapping resonances in longitudinal phase space.

Most importantly from the perspective of this dissertation, modulations pro- 
vide a frequency-dependent mechanism for detrapping particles captured within nonlinear resonance islands. This allows measurement of the island tune $Q_{\mathrm{I}}$ even in the experimental circumstance of large beam sizes. It is the objective of this chapter to describe this mechanism within the discrete Hamiltonian context and to summarize the requirements for both tune modulation and beta modulation to detrap particles captured near the resonance island fixed points.

The general character and sources of tune modulation and beta modulation are described in $\S 4.1$. The one-dimensional driven pendulum N-turn Hamiltonian with tune modulation is derived in $\S 4.2$, and the corresponding equations of motion are developed. In $\S 4.3$ we explore the structure of the tune modulation parameter space $\left(q, Q_{M}\right)$ and find four dynamical regions of interest - the adiabatic "amplitude-modulation" region, the fast-modulation "phase-modulation" region, the "strong-sideband" region and a region of chaos where the regular local motion around the fixed points vanishes. We investigate the effects of beta modulation in the N-turn Hamiltonian and compare them to those of tune modulation in $\S 4.4$, and compare the theoretical predictions of the previous sections to particle tracking for both modulations in $\S 4.5$.

\subsection{SOURCES OF TUNE MODULATION AND BETA MODULATION}

If $Q_{0}$ denotes the unperturbed tune, tune modulation is assumed to be of the form

$$
Q_{0} \rightarrow Q_{0}+q \sin \left(2 \pi Q_{M} t\right)
$$

The tune modulation strength, or depth, is $q$, and $Q_{M}$ is the modulation frequency; both are frequency-domain parameters and are expressed in inverse turns. This type of tune modulation unavoidably arises from two sources: ripple on quadrupole power supplies and coupling of synchrotron oscillations to the tunes via 
chromaticity. The latter is normally the more important source due to the conservative bounds placed on power supply current ripple, on the order of $\Delta I / I \approx 10^{-5}$ to stabilize machine tunes to $10^{-3}$ accuracy.

With typical chromaticities of several units and fractional momentum offsets $\delta$ of about $10^{-4}$, Equation (2.19) shows that modulation strengths $q$ of approximately $10^{-3}$ are always present in the Tevatron. The modulation frequency for these oscillations is the synchrotron frequency, ranging from $1.4 \cdot 10^{-3}$ at injection to $5.7 \cdot 10^{-4}$ at collision energies. It is difficult to reduce the momentum spread of the beam, both because of demands on RF systems and because of coupled bunch instabilities that appear at high longitudinal beam densities. Reduction of chromaticities $\xi$ is also impractical because chromaticities much smaller than a few units can cause strong head-tail instabilities in individual beam bunches.

Tune modulation can also be explicitly introduced by modulating the power supply currents of a set of quadrupole correctors. A modulated quadrupole error of strength $\Delta \tilde{b}_{1}$ at a location with horizontal beta function $\beta_{x}$ (quad) will modulate the horizontal tune vis. Equation (2.22):

$$
q=\frac{\Delta \tilde{b}_{1} \beta_{x}(\mathrm{quad})}{4 \pi}
$$

The vertical tune is modulated with an opposite sign (completely out of phase from the horizontal) using the corresponding beta function. Discussion of controlled modulation for the E778 tune modulation experiment is deferred until Chapter 5.

Quadrupole modulation not only changes the tune of the machine but also modulates the beta functions around the ring. Quantitatively the amplitude $b(s)$ of this beta modulation,

$$
\beta \rightarrow \beta\left[1+b(s) \sin \left(2 \pi Q_{M} t\right)\right],
$$

is given by Sands (Sands 1970, eq. [2.105]):

$$
b(s) \equiv \frac{\Delta \beta(s)}{\beta(s)}=\frac{2 \pi q}{\sin \left(2 \pi Q_{0}\right)} \cos \left[2 \mid \psi(s)-\psi(\text { quad }) \mid-2 \pi Q_{0}\right] .
$$


The only longitudinal position dependence is in the cosine; this perturbation causes a so-called "beta wave" around the accelerator with a cusp at the location of the quadrupole error. Examination of the first-order nonlinear Hamiltonians Equations (3.6) and (3.7) shows that, for the single resonance term $\cos (N \psi)$ selected by N-turn summation, the final effect of beta modulation is, to first order in $b$, a modulation of the resonance strength:

$$
V_{N} \rightarrow V_{N}\left[1+\frac{N b}{2} \sin \left(2 \pi Q_{M} t\right)\right]
$$

The beta modulation strength in Equation (4.4) is expressed in terms of the tune shift for the quadrupole to compare the relative effectiveness of tune and beta modulation in detrapping particles captured within nonlinear resonance islands. For a single quadrupole error the maximum beta modulation amplitude $b$ is roughly an order of magnitude larger than the corresponding tune modulation depth $q$; for a set of modulated quadrupoles distributed in phase $\psi(s)$ (as used in E778) interference between beta waves from individual quadrupoles makes this ratio about two to three times smaller.

A similar, though not equivalent, modulation is produced by synchrotron oscillations of a particle with nonzero fractional momentum offset $\delta$. These modulate the magetic rigidity $|B \rho| \rightarrow|B \rho|\left[1+\delta \sin \left(2 \pi Q_{M} t\right)\right]$, effectively modulating the normalized multipole strengths. For $\delta \ll 1$, always the case in realistic operations,

$$
\tilde{b}_{n} \rightarrow \tilde{b}_{n}\left[1-\delta \sin \left(2 \pi Q_{M} t\right)\right]
$$

to first order in $\delta$. This modulates the resonance strength $V_{N}$ differently than beta modulation does, since here $V_{N}$ is proportional to $\tilde{b}_{n}$ in a first-order nonlinear analysis:

$$
V_{N} \rightarrow V_{N}\left[1-\delta \sin \left(2 \pi Q_{M} t\right)\right]
$$

So $b=2 \delta / N$ for beta modulation induced by synchrotron oscillations. 


\subsection{THE N-TURN HAMILTONIAN FOR TUNE MODULATED ONE-DIMENSIONAL RESONANCES}

We begin with the one-turn one-dimensional Hamiltonian of Equation (3.13), including tune modulation:

$$
H_{1}(\psi, J ; t)=2 \pi Q_{0} J+\frac{1}{2} \alpha J^{2}+V_{N} J^{N / 2} \cos (N \psi)+2 \pi q J \sin \left(2 \pi Q_{M} t\right)
$$

The single resonance term is the only one kept because all others, not of interest in an isolated resonance model, are suppressed in the summation used to produce an N-turn Hamiltonian. The tune is again assumed to be near the resonant tune, $Q_{0}=\frac{M}{N}+\delta_{Q}$ where $\delta_{Q} \ll \frac{1}{N}$. We shall consider only tune modulation frequencies $Q_{M}$ much smaller than $1 / N$ so the tune is adiabatically changing over these $\mathrm{N}$ turns, and tune modulation depths $q \lesssim \delta_{Q}$. Real sources of tune modulation described in the previous section agree with these limits; much larger tune modulation strengths prohibit any regular motion with strongly driven resonances. The one-turn Hamiltonian is now summed over $N$ turns as in the previous chapter to give, to first order in the small strengths $\alpha, V_{N}$ and $q$ :

$$
\begin{aligned}
H_{N}(\psi, J ; T)= & 2 \pi N \delta_{Q} J+2 \pi N q J \sin \left(2 \pi N Q_{M} T\right)+\frac{N}{2} \alpha J^{2} \\
& +N V_{N} J^{N / 2} \cos (N \psi) .
\end{aligned}
$$

The N-turn Hamiltonian can exhibit fixed points as before for certain sections of the modulation parameter space $\left(q, Q_{M}\right)$. Solving the equation $\dot{J}=0$ for the fixed point phases gives $\phi_{f p}=0, \pi / N, \ldots(2 N-1) \pi / N$. Similarly, solving $\dot{\psi}=0$ for the fixed point actions gives

$$
\begin{aligned}
0 & =2 \pi \delta_{Q}+2 \pi q \sin \left(2 \pi N Q_{M} T\right)+\alpha J_{f p}, \\
\dot{J}_{f p} & =-\frac{4 \pi^{2} N}{\alpha} q Q_{M} \cos \left(2 \pi N Q_{M} T\right) .
\end{aligned}
$$

We transform the coordinates to the small action-angle coordinates $(\theta, I)$, expanded around a stable fixed point, via the linear transformations of the previous 
chapter -

$$
\begin{aligned}
& \phi=\frac{\pi}{N}+\theta, \\
& J=J_{f p}(T)+I .
\end{aligned}
$$

This transformation also changes the Hamiltonian because it is nonautonomous, or time-dependent:

$$
H_{N}(\psi, J ; T) \rightarrow H_{N}(\theta, I ; T)+\theta \dot{J}_{f p}
$$

Performing this change of coordinates produces a driven pendulum Hamiltonian,

$$
H_{N}(\theta, I)=\frac{N}{2} \alpha I^{2}+N V_{N} J_{f p}^{N / 2} \cos (N \theta)-\frac{4 \pi^{2} N}{\alpha} q Q_{M} \theta \cos \left(2 \pi N Q_{M} T\right),
$$

The equation of motion of the angle variable $\theta$ becomes that of a pendulum, driven at the modulation frequency $Q_{M}$ :

$$
\ddot{\theta}=-N\left(2 \pi Q_{\mathrm{I}}\right)^{2} \sin (N \theta)-4 N^{2} \pi^{2} q Q_{M} \cos \left(2 \pi N Q_{M} T\right) .
$$

This form agrees with others previously published (Peggs 1988, Chen 1990) if the timescale is changed back to single turns via $T \rightarrow t / N$.

When the equation of motion for the angle $\theta$ is linearized, it can be solved easily and explicitly for $\theta(T)$. The solution is found to be

$$
\theta(T)=\frac{q}{Q_{M}} \frac{Q_{M}^{2}}{Q_{M}^{2}-Q_{\mathrm{I}}^{2}} \cos \left(2 \pi Q_{M} N T\right) .
$$

From the Hamiltonian we can also get the solution for the action $I$ as a function of $T$, since $\dot{\theta} \approx N \alpha I$ :

$$
I(T)=\frac{2 \pi q}{\alpha} \frac{Q_{M}^{2}}{Q_{\mathrm{I}}^{2}-Q_{M}^{2}} \sin \left(2 \pi Q_{M} N T\right) .
$$

\subsection{STRUCTURE OF THE $\left(q, Q_{M}\right)$ PARAMETER SPACE}

From the structure of the driven pendulum equation of motion, it is natural to investigate the structure of the $\left(q, Q_{M}\right)$ modulation parameter space. Rescaling 
the time by $T \rightarrow T / Q_{\mathrm{I}}$ in Equation (4.14) shows that the natural scaling for both $q$ and $Q_{M}$ is $Q_{\mathrm{I}}$, since only the ratios $q / Q_{\mathrm{I}}$ and $Q_{M} / Q_{\mathrm{I}}$ appear under this scale change:

$$
\ddot{\theta}=-N(2 \pi)^{2} \sin (N \theta)-4 N^{2} \pi^{2}\left(\frac{q}{Q_{\mathrm{I}}}\right)\left(\frac{Q_{M}}{Q_{\mathrm{I}}}\right) \cos \left[2 \pi N\left(\frac{Q_{M}}{Q_{\mathrm{I}}}\right) T\right] .
$$

It is interesting to look at three extremes of the parameter space: when $Q_{M} \ll Q_{\mathrm{I}}$ (adiabatic modulation), $Q_{M} \gg Q_{\mathrm{I}}$ (fast modulation) and $Q_{M} \approx Q_{\mathrm{I}}$ (resonance).

First consider the case of adiabatic tune modulation, where the modulation tune is much smaller than the island tune, or many particle oscillations take place around the fixed point with every modulation cycle. Here we can consider the tune to be changing linearly with a small rate of $\dot{Q}=d Q / d T$. The transformed Hamiltonian of Equation (4.13) is now given by

$$
H_{N}(\theta, I ; T)=\frac{N}{2} \alpha I^{2}+N V_{N} J_{f p}^{N / 2} \cos (N \theta)+2 \pi \dot{Q} T I
$$

The dependence on $\dot{Q} T$ may be relegated to second order with the generating function

$$
G(I, \bar{\theta} ; T)=-I \bar{\theta}+\frac{2 \pi \dot{Q}}{\alpha} T \bar{\theta}
$$

which gives the transformations $\bar{I}=I-2 \pi \dot{Q} T / \alpha$ and $\theta=\bar{\theta}$. The new Hamiltonian is now no longer periodic in the angle variable $\bar{\theta}$, for it becomes

$$
H_{N}(\bar{\theta}, \bar{I})=\frac{N}{2} \alpha \bar{I}^{2}+N V_{N} J_{f p}^{N / 2} \cos (N \bar{\theta})+\frac{2 \pi \dot{Q}}{\alpha} \bar{\theta} .
$$

This Hamiltonian explicitly modulates the pendulum amplitude $\bar{I}$, as can be seen by the Hamilton's equation for it's rate of change:

$$
\dot{\bar{I}}=-\frac{\partial H_{N}}{\partial \bar{\theta}}=N^{2} V_{N} J_{f p}^{N / 2} \sin (N \bar{\theta})+\frac{2 \pi \dot{Q}}{\alpha} .
$$

Now there are only angle fixed points if we can find $\bar{\theta}_{f p}$ such that $\dot{\bar{I}}=0$. Noting that the maximum value of the rate of change of the tune is $\dot{Q}_{\max }=2 \pi N q Q_{M}$, 
we find the constraint

$$
\left|\frac{q}{Q_{\mathrm{I}}} \frac{Q_{M}}{Q_{\mathrm{I}}}\right|<\frac{1}{N}
$$

for adiabatic stability over the entire modulation sweep. As has been previously mentioned (Peggs 1988, Chao and Month 1974), this is an analogue to RF bucket shrinkage during particle acceleration. The above procedure is equivalent to transforming the original N-turn Hamiltonian (4.9) via a generating function that modulates only the action (or amplitude),

$$
G(\psi, I ; T)=\left[J_{f p}(T)+I\right]\left[\psi-k_{r e s} \pi\right]
$$

hence this region is dominated by "amplitude modulation", where the modulation predominantly modulates island amplitudes instead of island phases as mentioned above.

Second, consider the "fast modulation" case, where $Q_{M} \gg Q_{\mathrm{I}}$. As might be expected from the above comments, this region is dominated by "phase modulation." Consider transforming to the phase-modulated fixed points with application of the generating function

$$
G_{2}(\psi, I ; T)=\left[J_{f p}+I\right]\left[\psi-\psi_{f p}(T)\right]
$$

to the N-turn Hamiltonian (4.9) — this produces

$$
H_{N}(\theta, I)=\frac{N}{2} \alpha I^{2}+2 \pi N q I \sin \left(2 \pi N Q_{M} T\right)+N V_{N} J_{f p}^{N / 2} \cos (N \theta) .
$$

Here the phase is explicitly modulated by the term linear in the action $I$, as expected. Applying yet another generating function,

$$
G_{2}(\theta, \bar{I} ; T)=\theta \bar{I}+\frac{q}{Q_{M}} \cos \left(2 \pi N Q_{M} T\right) \bar{I}
$$

shifts the modulation inside the angle dependence:

$$
H_{N}(\bar{\theta}, \bar{I} ; T)=\frac{N}{2} \alpha \bar{I}^{2}+N V_{N} J_{f p}^{N / 2} \cos \left[N \bar{\theta}+\frac{N q}{Q_{M}} \cos \left(2 \pi Q_{M} N T\right)\right] .
$$


The cosine term can be expanded in Bessel functions $J_{m}$ and harmonics via a variant of the Jacobi-Anger expansion of a plane wave,

$$
\cos (A+B \cos C)=\sum_{m=-\infty}^{\infty} J_{m}(B) \cos (A+m C+m \pi / 2)
$$

to produce a new Hamiltonian that has an infinite number of resonance terms:

$H_{N}(\bar{\theta}, \bar{I})=\frac{N}{2} \alpha \bar{I}^{2}+N V_{N} J_{f p}^{N / 2} \sum_{m=-\infty}^{\infty} J_{m}\left(\frac{N q}{Q_{M}}\right) \cos \left(N \bar{\theta}+2 \pi m N Q_{M} T+m \pi / 2\right)$.

Examine a particle at non-zero $\bar{I}$ in the above Hamiltonian; there is a family of actions $\bar{I}_{l}$ (parameterized by an integer $l$ ) where a particle has a net phase advance of zero (modulo 1 ) over $N T_{M}$ turns, or a tune of

$$
Q_{l}=\frac{M}{N}+\frac{l}{N} Q_{M}
$$

The action that corresponds to this tune is $\bar{I}_{l}=\frac{2 \pi l Q_{M}}{N \alpha}$. Now we can perform a sum over $T_{M}$ N-turn periods near this tune if not much happens in that time (if $Q_{M} \ll Q_{\mathrm{I}}$ ) to find a new Hamiltonian that is autonomous over discrete time intervals of $T_{M} \cdot N$ turns. When this sum is performed, only one resonance $(m=$ $-l)$ remains since the others are suppressed in a similar manner to the resonance suppression before, and we find the Hamiltonian for $\left(T_{M} \cdot N\right)$-turn motion:

$$
H_{N \cdot T_{M}}(\bar{\theta}, \bar{I})=\frac{N}{2} T_{M} \alpha\left(\bar{I}-\bar{I}_{l}\right)^{2}+N V_{N} J_{f p}^{N / 2} T_{M} J_{-l}\left(\frac{N q}{Q_{M}}\right) \cos (N \bar{\theta}) .
$$

Note that this Hamiltonian contains a sideband resonance for every integer $l$; however the Bessel functions suppress the amplitudes of these sideband resonance strengths for large $l$ since $J_{-l} \approx 0$ for $N q / Q_{M}<|l|$. For the unsuppressed sidebands the Bessel functions can be approximated by

$$
J_{-l}\left(\frac{N q}{Q_{M}}\right) \approx\left(\frac{2 Q_{M}}{N \pi q}\right)^{1 / 2} \cos \left(\frac{N q}{Q_{M}}+\frac{l \pi}{4}-\frac{\pi}{4}\right), \quad \text { for }|l|<\frac{N q}{Q_{M}}
$$


with rms values $\sqrt{Q_{M} / N \pi q}$. This means that the $l=1$ sideband suppressed and no sidebands are driven strongly if

$$
q<\frac{Q_{M}}{N}
$$

Generally the $k^{t h}$ sideband is suppressed if $q<k Q_{M} / N$.

From the Chirikov overlap criterion (Chirikov 1979), sideband resonances overlap and local stochasticity results if $2 I_{W}>I_{\text {sep }}$, where $I_{W}$ is the sideband resonance island action half-width and $I_{\text {sep }}$ is the action separation of the fixed points of neighboring sidebands. From the above formula for $\bar{I}_{l}$, it is apparent that $I_{\text {sep }}=2 \pi Q_{M} / N \alpha$. The island width must be calculated from Hamiltonian contour considerations and Equation (4.31) in the same fashion as the unperturbed primary resonance width calculation in Chapter 3 ; here it is found to be

$$
I_{W}=2\left[\frac{V_{N} J_{f p}^{N / 2}}{\alpha} J_{-l}\left(\frac{N q}{Q_{M}}\right)\right]^{1 / 2} \approx 2\left[\frac{V_{N} J_{f p}^{N / 2}}{\alpha}\right]^{1 / 2}\left[\frac{Q_{M}}{N \pi q}\right]^{1 / 4}
$$

for each sideband. Combining these with the overlap criterion and the definition of $Q_{\mathrm{I}}$ for the primary resonance (Equation [3.21]), sidebands overlap and chaos begins when

$$
256 Q_{\mathrm{I}}^{4}>N \pi q Q_{M}^{3}
$$

Even though separatrices are destroyed and sidebands overlap when this condition is obeyed, the central regions of the primary resonances can still be locally stable for small enough $Q_{M}$, where adiabatic trapping takes over as in the previous discussion.

Lastly consider the nearly resonant case, $Q_{M} \approx Q_{\mathrm{I}}$, as described approximately by the linearized equations of motion. Equations (4.15) and (4.16) represent locally phase-locked motion only when the linearization of this motion around the fixed point in phase is a good approximation. This approximation fails when 
$\sin (N \theta)$ becomes strongly nonlinear — that is, when $|N \theta| \approx 1$. This gives a weak constraint for bounded motion as

$$
\left|\frac{q Q_{M}}{Q_{M}^{2}-Q_{I}^{2}}\right|<1 / N
$$

This constraint can be improved by including the next order (nonlinear) terms in the expansion of the driven pendulum equation (Tsironis 1990) but it is satisfactory in the resonant region where $Q_{M} \approx Q_{\mathrm{I}}$ and large motion is expected to become unbounded.

Summarizing, there are four basic constraints in the parameter space that divide the $\left(q, Q_{M}\right)$ plane into four distinct regions. Near the driven pendulum resonance $\left(Q_{M} \approx Q_{\mathrm{I}}\right)$, chaos occurs when

$$
\left|\frac{q Q_{M}}{Q_{M}^{2}-Q_{\mathrm{I}}^{2}}\right|>1 / N
$$

In the adiabatic regime $\left(Q_{M} \ll Q_{\mathrm{I}}\right)$, chaos occurs when

$$
\left|q Q_{M}\right|>\frac{Q_{I}^{2}}{N}
$$

below this threshold we have adiabatic stability of the fixed point and amplitude modulation dominates. In the fast-modulation approximation $\left(Q_{M} \gg Q_{\mathrm{I}}\right)$ the $k^{t h}$ sideband appears when $q>|k| Q_{M} / N$. The first sideband off the primary resonance is then of non-negligible size when

$$
q>\frac{Q_{M}}{N}
$$

below this threshold there is stability with fast "phase modulation". The sidebands overlap and produce chaos when

$$
256 Q_{\mathrm{I}}^{4}>N \pi q Q_{M}^{3}
$$




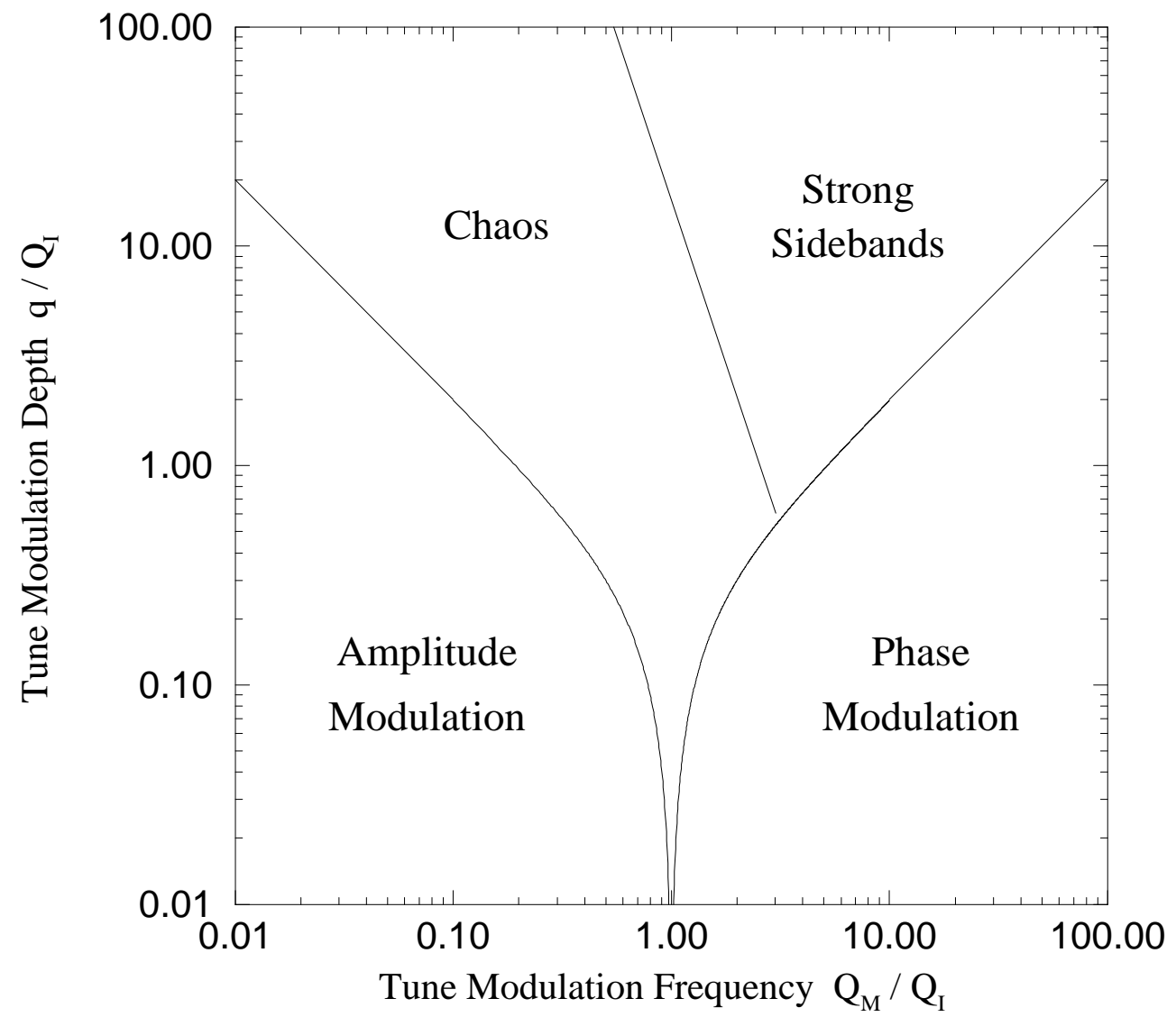

Figure 4.1: The $\left(q, Q_{M}\right)$ parameter plane for $N=5$, showing four distinct phases of motion. Local motion near the fixed point of the $5 Q_{x}$ resonance is stable (i.e. phase-locked, or resonant) for all regions except "Chaos".

These regions of the tune modulation parameter plane $\left(q, Q_{M}\right)$ and the lines separating them are shown in Figure (4.1).

The only tune modulation parameters that create detrapping of particles circling near the centers of nonlinear resonance islands are in the "Chaos" region of this figure, where there is expected to be no regular motion at all within the resonance island chain. It is important to note that with the presence of detuning and for purely one-dimensional motion, this chaotic region is localized. That is, the chaotic motion is restricted to a "thick layer" of stochasticity, covering the extent 
of the overlapping sidebands created by the modulation. The presence of such a region does not necessarily prohibit stable motion at larger amplitudes if the detuning that is present is sufficiently strong to pull the tunes of particles at these amplitudes to nonresonant values. This is a contrast to "thin-layer" stochasticity, which is created by the overlap of thinner higher-order resonances in the vicinity of perturbed separatrices. For our purposes such thin-layer stochasticity can be ignored in this discussion, since the phase space extent of such regions is a great deal smaller than the extent of the chaotic band created by tune modulation.

The phase space of particles in each area of the tune modulation parameter plane is shown in Figure (4.2), plotted every modulation period. Shown on top for reference is the phase space of unperturbed particles, with $Q_{\mathrm{I}}=6.1 \cdot 10^{-3}$ for the primary isolated $N=5$ resonance shown. This is a nominally realistic value for $Q_{\mathrm{I}}$, also similar to values found for small nonlinear strengths in tracking in the last chapter. Note that motion in the amplitude modulation and phase modulation regions is essentially indistinguishable from the unperturbed phase space and small-amplitude nonresonant motion is undisturbed in all cases. In the lower right figure the $k=1$ sidebands can be seen on either side of the primary resonance, with stochasticity already beginning to form where the separatrices of the resonances overlap.

Alternative schemes to investigating the stability of the driven pendulum equation of motion (4.14) have been proposed which do not linearize the pendulum completely but including the first nonlinear terms in the expansion of the sine (Tsironis, Peggs and Chen 1990). Such an analysis predicts stability boundaries of the Mathieu equation, similar to the discussion of beta modulation in the next section. However these resonances are expected to be significantly weaker than the main driven pendulum resonance because they are found within the nonlinear response of the pendulum system. 

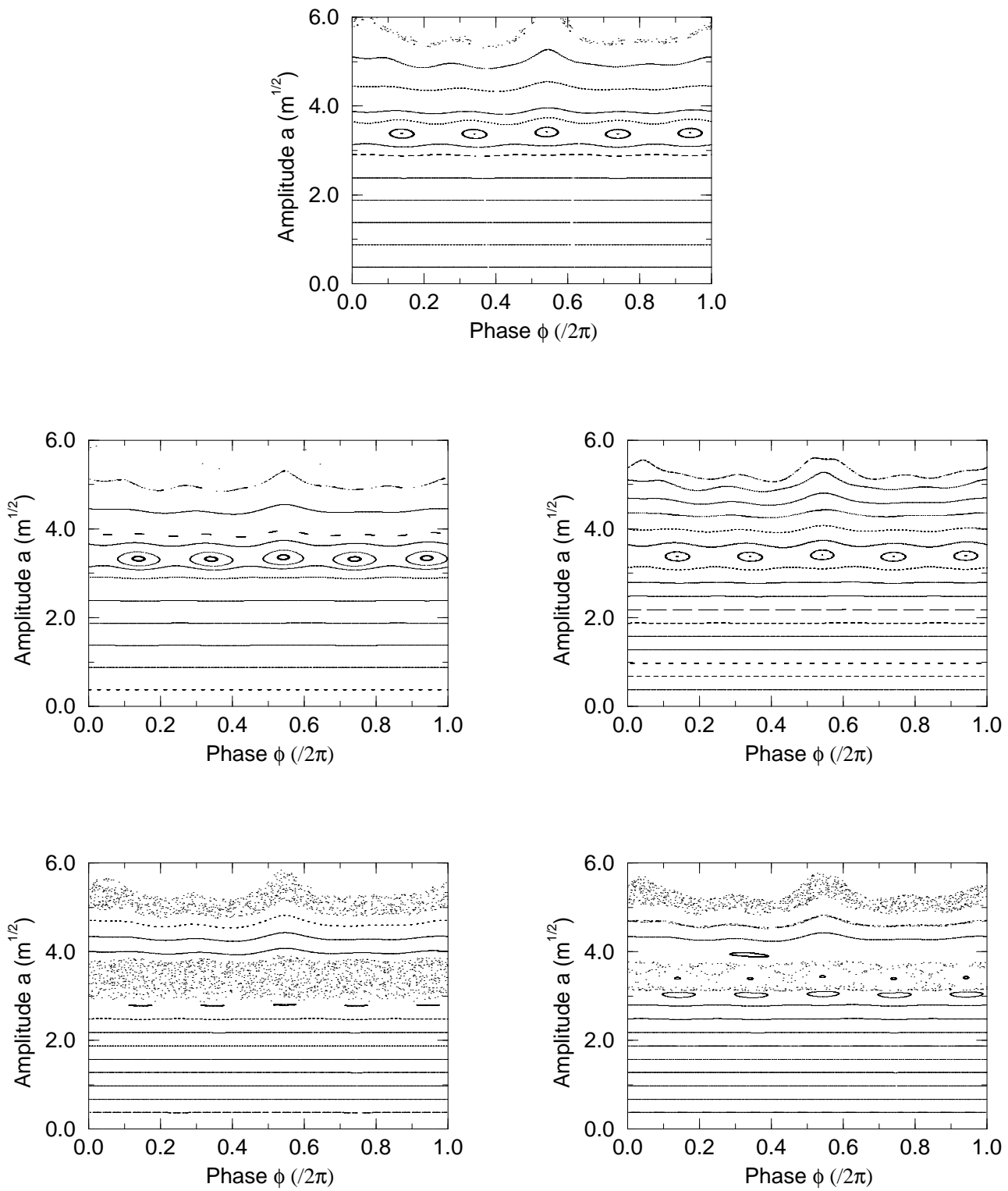

Figure 4.2: Phase spaces at various points in the $\left(q, Q_{M}\right)$ tune modulation parameter plane as produced by the simulation program OdTrack for $N=5$ resonance islands. Here the action $a=\sqrt{2 J}$ is plotted against particle phase, for $Q_{\mathrm{I}}=6.08 \cdot 10^{-3}$. From the top and left to right, the figures are described as: (a) The unperturbed phase space at $q=Q_{M}=0$. (b) Amplitude modulation with $q=Q_{M}=Q_{\mathrm{I}} / 10$. (c) Phase modulation with $q=Q_{\mathrm{I}} / 10, Q_{M}=10 Q_{\mathrm{I}}$. (d) A thick stochastic band is produced in the chaos region with $q=Q_{\mathrm{I}} / 2, Q_{M}=Q_{\mathrm{I}}$. (e) The first sideband $(k=1)$ appear at $q=2 Q_{\mathrm{I}} / 5, Q_{M}=Q_{\mathrm{I}} / 3$. 


\subsection{THE N-TURN HAMILTONIAN FOR BETA MODULATED 1-DIMENSIONAL RESONANCES}

Now consider the one-turn one-dimensional Hamiltonian of Equation (3.13), representing the motion of a particle trapped in a nonlinear resonance island influenced by a small beta modulation (4.5):

$$
H_{1}(\psi, J ; t)=2 \pi Q J+\frac{1}{2} \alpha J^{2}+V_{N} J^{N / 2} \cos (N \psi)\left[1+\frac{N b}{2} \sin \left(2 \pi Q_{M} t\right)\right] .
$$

With the assumption that the tune is near an $N Q$ resonance as before, quantified by stating that $Q=M / N+\delta_{Q}$, we can now go through the N-turn summing process to arrive at an N-turn version of this Hamiltonian:

$$
\begin{aligned}
H_{N}(\psi, J ; T)= & 2 \pi N \delta_{Q} J+\frac{N}{2} \alpha J^{2} \\
& +N V_{N} J^{N / 2} \cos (N \psi)\left[1+\frac{N b}{2} \sin \left(2 \pi Q_{M} N T\right)\right] .
\end{aligned}
$$

The equations of motion given by this Hamiltonian show that neither the fixed point action nor phase are modulated by beta modulation - it is the depth of the oscillator well, or resonance strength, which is being modulated. Transforming to the coordinates $(\theta, I)$ is then not time-dependent, and can be accomplished using the linear canonical transformation of Equation (3.17). The Hamiltonian after this transformation has the form of a parametrically modulated nonlinear pendulum,

$$
H_{N}(\theta, I ; T)=\frac{N}{2} \alpha I^{2}+N V_{N} J_{f p}^{N / 2} \cos (N \theta)\left[1+\frac{N b}{2} \sin \left(2 \pi Q_{M} N T\right)\right] .
$$

Comparison of the relative effectiveness of tune modulation and beta modulation in detrapping particles within resonance islands and destroying persistent signals is more straightforward if we compare the respective equations of motion. The equation of motion for the angle of this oscillator in all ranges of modulation strength $b$ and frequency $Q_{M}$ is then

$$
\ddot{\theta}=-N\left(2 \pi Q_{\mathrm{I}}\right)^{2} \sin (N \theta)\left[1+\frac{N b}{2} \sin \left(2 \pi Q_{M} N T\right)\right] .
$$


The parametric form of the equation of motion is expected in the case of beta modulation because the strength of the resonance is being modulated as mentioned above, thus modulating both the island tune $Q_{\mathrm{I}}$ and the island width. The fixed point phases are not expected to change with beta modulation to any order, as can be seen by examining the above equation of motion when $\theta=0$. From Equation (4.41) the equation for the fixed point action $J_{f p}$ is

$$
0=2 \pi Q+\alpha J_{f p}+\frac{N}{2} V_{N} J^{\frac{N-2}{2}}\left[1+\frac{N b}{2} \sin \left(2 \pi Q_{M} N T\right)\right],
$$

indicating that this action is modulated in some complicated weak way.

The linearized form of Equation (4.44) is called the Mathieu equation. Conditions for the stability of such a parametrically driven oscillator have been described extensively in classical mechanics literature (Landau and Lifshitz 1975) and various tables of stability curves have been produced (Abromowitz and Stegun 1965, McLachlan 1951). The above form can be transformed to the canonical form cited in the literature,

$$
\frac{d^{2} \theta}{d z^{2}}+[a-2 f \sin (2 z)] \theta=0
$$

noting the equivalences $z=N \pi Q_{M},\left(Q_{M} / Q_{\mathrm{I}}\right)=2 / a^{1 / 2}$, and $b=\frac{f}{2 N}\left(\frac{Q_{M}}{Q_{\mathrm{I}}}\right)^{2}$. Note that the modulation frequency still scales naturally with the island frequency, but the modulation strength $b$ usurprisingly does not because it is not a natural frequency domain variable. For values of $b \ll 1$, resonances exist for the betamodulated parametric oscillator when

$$
Q_{M}(\text { resonant })=\frac{2 Q_{\mathrm{I}}}{k}
$$

where $k$ is an positive integer. The strength of these resonances increases with increasing modulation strength $b$, but decreases with increasing $k$ as $b^{k}$; the subharmonic resonance at $k=1$ ( or $Q_{M}=2 Q_{\mathrm{I}}$ ) is the strongest. 


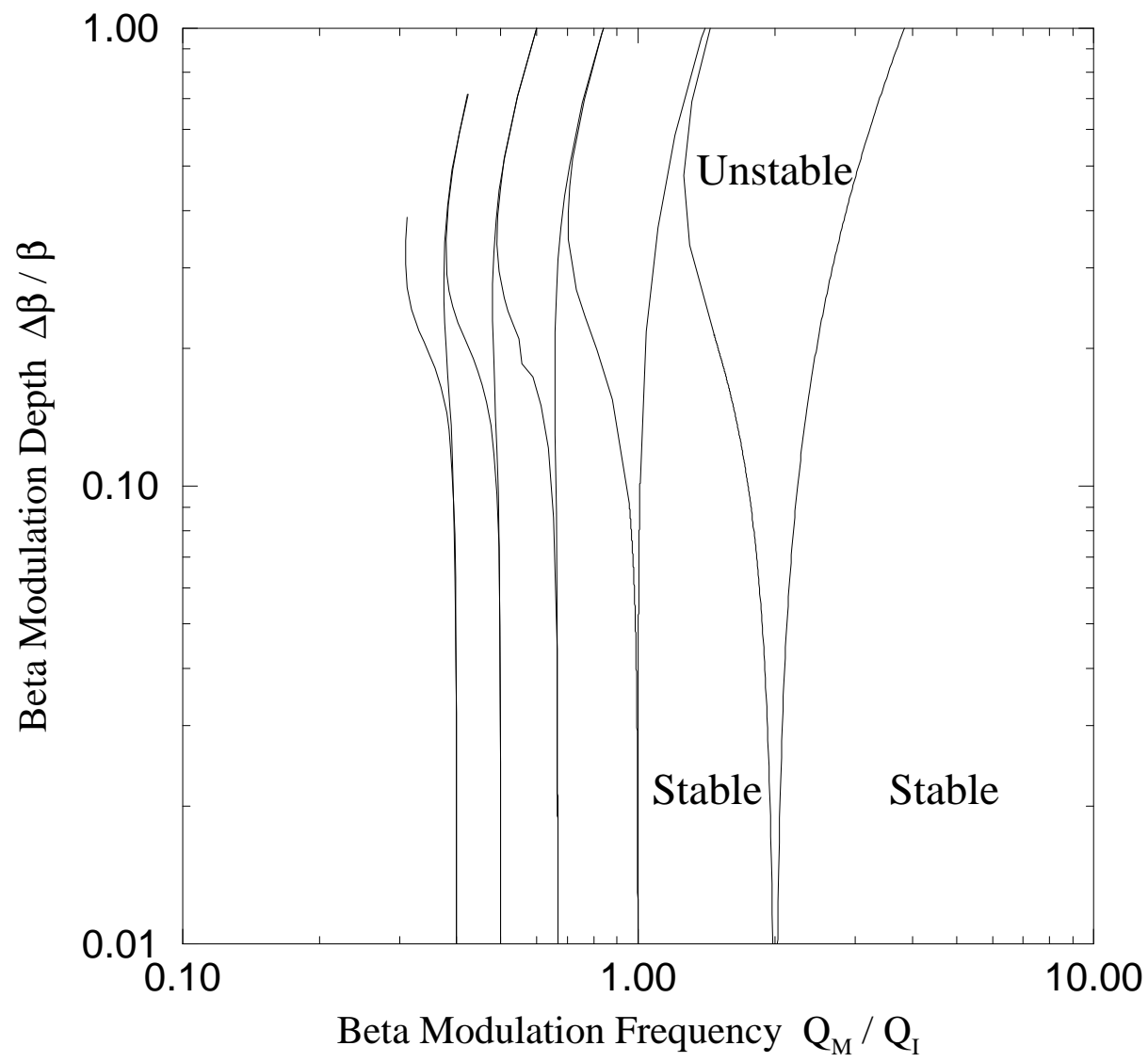

Figure 4.3: The $N=5$ modulation parameter plane $\left(b, Q_{M} / Q_{\mathrm{I}}\right)$ for beta function modulation, showing approximate widths for resonances of orders $k=1$ to $k=5$. Small oscillation stability is predicted below each set of resonance lines.

The $k=1$ through $k=5$ Mathieu resonances are shown in Figure (4.3), where motion within a resonance is expected to lead to instability of motion very close to the fixed point and subsequent detrapping. The $k=1$ subharmonic resonance is evident at $Q_{M}=2 Q_{\mathrm{I}}$. All of the resonance widths typically grow non-negligible at a beta modulation depth of approximately $b \approx 0.10$. Higher order resonances are present but not shown for the sake of clarity. Note that in this stability diagram the vertical scale is $b=\frac{\Delta \beta}{\beta}$. Comparison of this figure to Figure (4.1) and recalling comments about the relative strengths of beta modulation and tune 
modulation induced by a quadrupole error shows that tune modulation is on the order of $1 /\left(10 Q_{\mathrm{I}}\right)$ times more effective than beta modulation in destroying local regular motion near the center of a nonlinear resonance island.

\subsection{COMPARING HAMILTONIAN RESULTS TO SIMULATION}

The entirety of this chapter concerns the phase-locking "stability" of particles trapped within nonlinear resonance islands subject to tune modulation and beta modulation. It is quite tedious to examine phase-space plots by hand for a variety of modulation conditions to determine the stability of the fixed-points, and so an algorithm was developed that allows fast and efficient location of fixed points in the two-dimensional map of the octupole-decapole lattice. This algorithm is quite easily extendible to any two-dimensional map.

Consider a fixed point in the phase space $\left(x, x^{\prime}\right)$ at the location $\left(\bar{x}, \bar{x}^{\prime}\right)$. If this fixed point is elliptically stable a particle circulating around it will experience the linear transformation from initial coordinates $\left(x, x^{\prime}\right)$ to $\left(x+\Delta x_{1}, x^{\prime}+\Delta x_{1}^{\prime}\right)$

$$
\left(\begin{array}{c}
x+\Delta x_{1} \\
x^{\prime}+\Delta x_{1}^{\prime}
\end{array}\right)=\left(\begin{array}{c}
\bar{x} \\
\bar{x}^{\prime}
\end{array}\right)+\left(\begin{array}{cc}
A & B \\
C & D
\end{array}\right)\left(\begin{array}{c}
x-\bar{x} \\
x^{\prime}-\bar{x}^{\prime}
\end{array}\right),
$$

or

$$
\left(\begin{array}{c}
x+\Delta x_{1} \\
x^{\prime}+\Delta x_{1}^{\prime}
\end{array}\right)=\left(\begin{array}{c}
A x-A \bar{x}+B x^{\prime}-B \bar{x}^{\prime}+\bar{x} \\
C x-C \bar{x}+D x^{\prime}-D \bar{x}^{\prime}+\bar{x}^{\prime}
\end{array}\right) .
$$

The transformation matrix has the form of a rotation matrix if the motion around the fixed point is perfectly circular. For a more general elliptical fixed point, the trace of the rotation matrix is twice the cosine of the the total phase advance induced by the mapping; if this phase advance is denoted $2 \pi Q_{\mathrm{I}} T$, where $T$ is the number of turns tracked to produce one iteration of this map in Odfp, then

$$
\cos \left(2 \pi Q_{\mathrm{I}} T\right)=\frac{A+D}{2}
$$


This determines $Q_{\mathrm{I}}$ within an aliasing factor from the cosine once the transformation matrix elements are known, and this aliasing factor can be found by comparison of this $Q_{\mathrm{I}}$ to the theoretically predicted value.

Equation (4.49) only gives two constraints to find six unknowns, the fixed point location and the elements of the transformation matrix. Four other constraints are given by launching two more particles through the same mapping, offset by small amounts $\delta x$ and $\delta x^{\prime}$ in the $x$ and $x^{\prime}$ directions respectively. The second particle experiences the linear transformation from $\left(x+\delta x, x^{\prime}\right)$ to $\left(x+\Delta x_{2}, x^{\prime}+\Delta x_{2}^{\prime}\right)$ :

$$
\left(\begin{array}{c}
x+\Delta x_{2} \\
x^{\prime}+\Delta x_{2}^{\prime}
\end{array}\right)=\left(\begin{array}{c}
A x-A \bar{x}+B x^{\prime}-B \bar{x}^{\prime}+\bar{x}+A \delta x \\
C x-C \bar{x}+D x^{\prime}-D \bar{x}^{\prime}+\bar{x}^{\prime}+C \delta x
\end{array}\right)
$$

Subtracting the mapping equations for the first particle from those of the second gives equations for the matrix elements $A$ and $C$ :

$$
A=\frac{\Delta x_{2}-\Delta x_{1}}{\delta x} \quad C=\frac{\Delta x_{2}^{\prime}-\Delta x_{1}}{\delta x} .
$$

Repeating the same process for a particle mapped from coordinates $\left(x, x^{\prime}+\delta x^{\prime}\right)$ to $\left(x+\Delta x_{3}, x^{\prime}+\Delta x_{3}^{\prime}\right)$ gives the other two matrix elements:

$$
B=\frac{\Delta x_{3}-\Delta x_{1}}{\delta x^{\prime}} \quad D=\frac{\Delta x_{3}^{\prime}-\Delta x_{1}^{\prime}}{\delta x^{\prime}} .
$$

Once the transformation matrix is known, Equation (4.49) can be inverted to find the fixed-points of the mapping:

$$
\left(\begin{array}{c}
\bar{x} \\
\bar{x}^{\prime}
\end{array}\right)=\left[1-\left(\begin{array}{cc}
A & B \\
C & D
\end{array}\right)\right]^{-1}\left[\left(\begin{array}{c}
x+\Delta x_{1} \\
y+\Delta x_{1}^{\prime}
\end{array}\right)-\left(\begin{array}{cc}
A & B \\
C & D
\end{array}\right)\left(\begin{array}{c}
x \\
x^{\prime}
\end{array}\right)\right] .
$$

This implies a matrix inversion - if this matrix inversion fails, then the initial conditions were such that the three particles were proceeding essentially linearly and there is no fixed point nearby.

This method has several advantages that make it extremely useful. It is extremely fast, requiring only three iteration about the local fixed point to assess 
local motion, as opposed to minmax fixed-point location techniques where many hundreds of iterations must be performed. It is also very efficient and accurate; only two or three iterations of this procedure were required to find the fixed point of a particle even launched near the separatrix. And finally, it is very robust; anywhere in the two-dimesional phase space where there is local curvature created by the presence of a resonance island, this method will iterate. If such curvature is produced by proximity of a stable fixed point, the method will converge; conversely, if such curvature is produced by proximity of an unstable fixed point, this method will rapidly diverge.

The program Odfp is used to monitor local stability of a $5 Q_{x}$ resonance island fixed point in the octupole-decapole lattice. For a given modulation tune $Q_{M}$, the fixed point was found for zero modulation strength. The modulation strength was then gradually increased and the local fixed point was again located, and the process was repeated until local motion was so distorted by the perturbation that no fixed point was found. This scan of modulation strengths was repeated for many tune modulation frequencies, and the points in the parameter space $\left(q, Q_{M}\right)$ where the fixed point disappeared were plotted. Consecutive iterations with a variety of modulation strengths makes it possible to extrapolate fixed point locations through vertically thin regions of instability in some cases. However, for large regions of chaos merely the edges can be examined, as the strongly chaotic motion precludes any chance of finding fixed points.

The results of such a set of simulations for the case of tune modulation, with beta modulation explicitly absent, are shown in Figure (4.4). A point is plotted for each $\left(q, Q_{M}\right)$ point where the fixed point was not found (or when local motion around the fixed point was not phase locked). The scan begins at $Q_{M}=0.1 Q_{\mathrm{I}}$, corresponding to a modulation period of approximately 1650 turns — searching further into the low-frequency adiabatic territory is constrained by available com- 


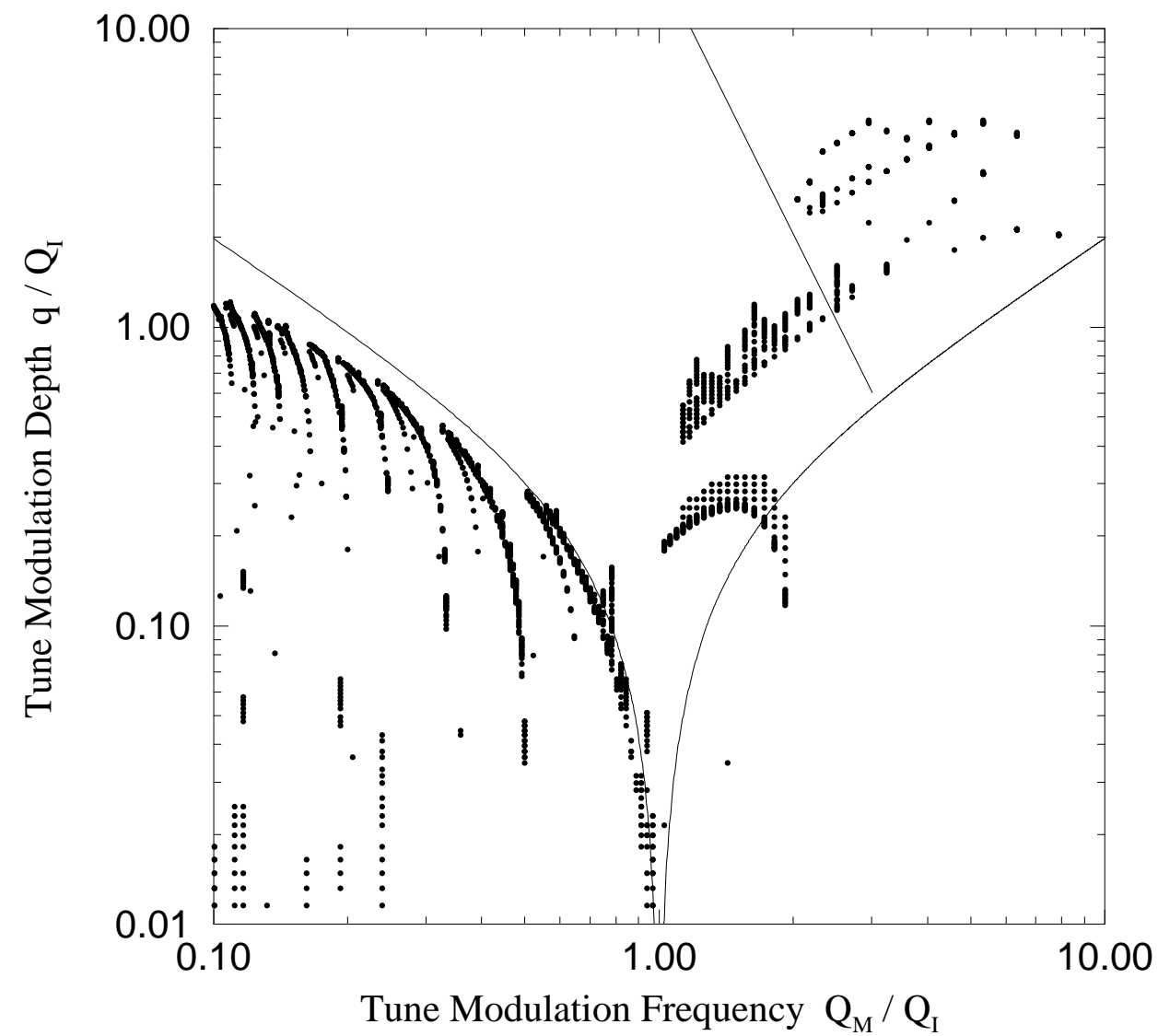

Figure 4.4: The $\left(q, Q_{M}\right)$ parameter plane for $N=5$, showing fixed point stability for a run of the tracking program Odfp. For this lattice $b_{3}=0.010, \tilde{b}_{4}=0.005, Q_{\mathrm{I}}=0.0061$ and $1 / Q_{\mathrm{I}} \approx 165$ turns. Lines from Figure (4.1) are shown for comparison to theory.

puter time and shows highly erratic behavior of many closely spaced resonances as described in the next paragraph. The frequency scan ends at $Q_{M}=10 Q_{\mathrm{I}}$, where the modulation period is approximately 16 turns and the approximation of modulation adiabaticity with respect to the turn time within the machine, $Q_{M} \ll 1$, begins to break down.

This figure shows the dominant $Q_{M}=Q_{\mathrm{I}}$ resonance, but also indicates that the stability border around this resonance is asymmetric. The low-frequency side of this resonance curves to low frequencies, and qualitatively agrees with the pre- 


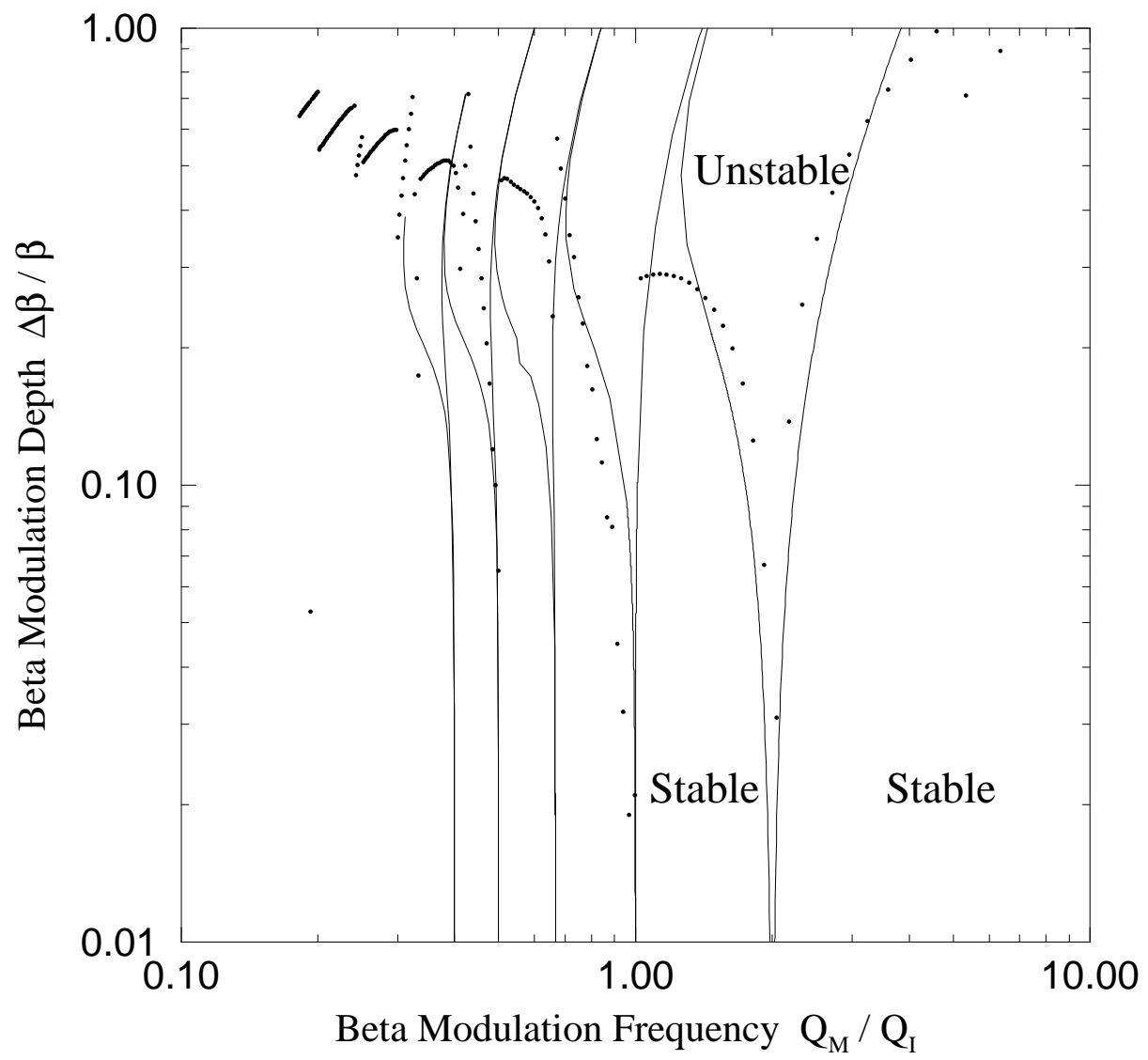

Figure 4.5: The $\left(b, Q_{M}\right)$ parameter plane for $N=5$, showing fixed point stability for a run of the tracking program Odfp. Tracking conditions were the same as those in Figure (4.4), and stability curves from Figure (4.2) are shown as solid lines for comparison.

dictions of tune modulation stability. However, the high-frequency side of this resonance appears almost vertical — at modulation frequencies just above the island tune, stability is greatly enhanced. This asymmetry is probably due to the higher order terms that have been neglected; indeed, the parametric oscillator displays such an asymmetry at this resonance (Landau and Lifshitz 1975). The presence of a cascade of thinner higher-order resonances at low frequencies, each dipping down at precisely the harmonics corresponding to Mathieu resonances, and the crescent-shaped instability region between $Q_{M}=Q_{\mathrm{I}}$ and the subhar- 
monic resonance $Q_{M}=2 Q_{\mathrm{I}}$ also indicate that the nonlinear driven pendulum equation of motion (4.14) has weak instabilities defined by the Mathieu equation, as predicted elsewhere (Tsironis, Peggs and Chen 1990). At higher frequencies stability is shown up to large modulation strengths, on the order of values that would make the assumption that the tune modulation depth $q$ is less than the difference between the base tune and the resonant tune false. There is indeed a large stable region in the area of "stable sidebands".

For beta function modulation a similar set of simulations were performed, with tune modulation absent, and the results of these simulations are shown in Figure (4.5). Resonance locations are exactly the same as predicted, though the behavior of tracking stability is somewhat different than that of the Mathieu predictions. This is quite reasonable because an approximation was made that $b \ll 1$ in the derivation of the Mathieu form for beta modulation, and so the predicted stability is expected to hold only for modulation levels much less than the beta function itself. Comparing the vertical scales between Figures (4.4) and (4.5) again shows that, for tune and beta modulation created by the same strength quadrupole ripple, tune modulation destroys the phase localization of resonance islands much more effectively. 


\section{CHAPTER 5 TUNE MODULATION AND EXPERIMENT E778}

Experiment E778, nonlinear dynamics in the Fermilab Tevatron, started in 1987 to answer several questions regarding nonlinear dynamics issues in accelerators, in particular how accurately the the linear aperture of an actual synchrotron compares to that predicted by design and simulation programs. Various type$\mathrm{s}$ of simulation programs have been used within the field of accelerator physics, both to assist in machine design and lattice modification. These simulations are extremely important in the design of new accelerators such as SSC, LHC, and RHIC, where performance demands typically requiring magnets with larger apertures and higher field quality must be balanced against the financial burden of construction.

Examination of the linear aperture is a more straightforward matter than examining the dynamic aperture, defined as the betatron oscillation amplitude which separates stable motion from unstable motion over some period of time. The dynamic aperture depends on many quantities, such as the physical aperture of the machine and the time period over which stability is being questioned. The linear and dynamic apertures depend strongly on nonlinearities, both intentionally and unintentionally introduced, in the synchrotron; it is therefore important to be able to model these nonlinearities with confidence for the sake of reducing magnet cost without sacrificing performance, and design correction schemes for nonlinearities that adversely affect the performance of these machines.

Prior to 1991 several experimental runs of E778 investigated the linearity of the Tevatron in normal operational mode (i.e. without any intentionally introduced strong nonlinearities) and such effects as smear, detuning and dynamic aperture 
as created by 16 controlled strong sextupoles. These portions of the experiment have been extensively documented elsewhere (Chao et. al. 1987b, Merminga 1989, Li 1990). Persistent signals due to resonant capture of a fraction of the beam were also used as an experimental beam diagnostic in E778, and comparison of kick amplitudes and persistent signal amplitudes allowed estimates of the island size $\delta I$.

In this chapter we describe the procedure and results of a tune modulation experiment performed in the Fermilab Tevatron in January, 1991. This experiment, a portion of Fermilab experiment E778, observed persistent signals in the horizontal transverse dimension corresponding to beam capture on a variety of resonances created by a set of strong sextupoles. The behavior of particles trapped in one of these resonances, the $5 Q_{x}$ resonance at the horizontal tune $Q_{x}=20.40$, was systematically examined under the influence of controlled tune modulation for two distinct nonlinear configurations and three different horizontal island amplitudes. For one particular case of sextupole configuration and island amplitudes a detailed analysis of the response of the persistent signal at high frequencies is found to agree with the one-dimensional tune modulation model presented in Chapter 4. A transverse diffusion experiment was also carried out during this experimental run but is not commented upon further here (Chen et. al. 1992).

The requirements for the E778 persistent signal and tune modulation experiment in the Tevatron are outlined in $\S 5.1$. Preparations for the January, 1991 experimental run are described in $\S 5.2$, including magnet calibrations and the results of preliminary tracking to determine which nonlinear magnet configurations should be used. The island tunes and detuning coefficients $\alpha$ are also measured from tracking of the configurations chosen for the experiment. The experimental run itself is described briefly in $\S 5.3$, and in $\S 5.4$ the data analysis procedure is explained and results of this experiment are summarized. 


\subsection{REQUIREMENTS FOR THE EXPERIMENT}

Several requirements must be fulfilled before a persistent signal and tune modulation experiment can be undertaken. These requirements fall under two general categories: the requirements of the accelerator lattice and the requirements of the data acquisition system.

\subsubsection{Lattice Requirements}

The requirements of the machine lattice are threefold: first, the lattice must be relatively linear with the exception of strong controlled nonlinearities, which are used to create resonance islands in transverse phase space. Without a relatively linear machine, other sources of nonlinearity will affect the resonance under study, possibly changing its size and position in phase space. Although this does not invalidate the experiment, the results of particle tracking and theoretical predictions are much less likely to compare favorably with experiment in the presence of unknown strong nonlinearities. It has previously been demonstrated that the nominal configuration of the Tevatron is exceptionally linear (Chao et. al. 1987b). Several unused sextupoles originally intended for harmonic correction are ideal for use as controlled nonlinearities. The sixteen sextupoles shown in Figure (6.1) were chosen for use in this experiment.

The accelerator must have available a fast kicker magnet in the transverse plane under study. This kicker must have a kick time less than the revolution period of the accelerator in order to kick the beam only once. Typically most accelerators have fast kickers such as these for abort systems, injection and fast extraction. The Tevatron has several such kickers; the one chosen for this experiment is the horizontal proton injection alignment kicker which is located at the Tevatron lattice location E17 and has a fall time of approximately 1-2 $\mu$ s, much smaller than the $21 \mu$ s revolution period of the Tevatron. 


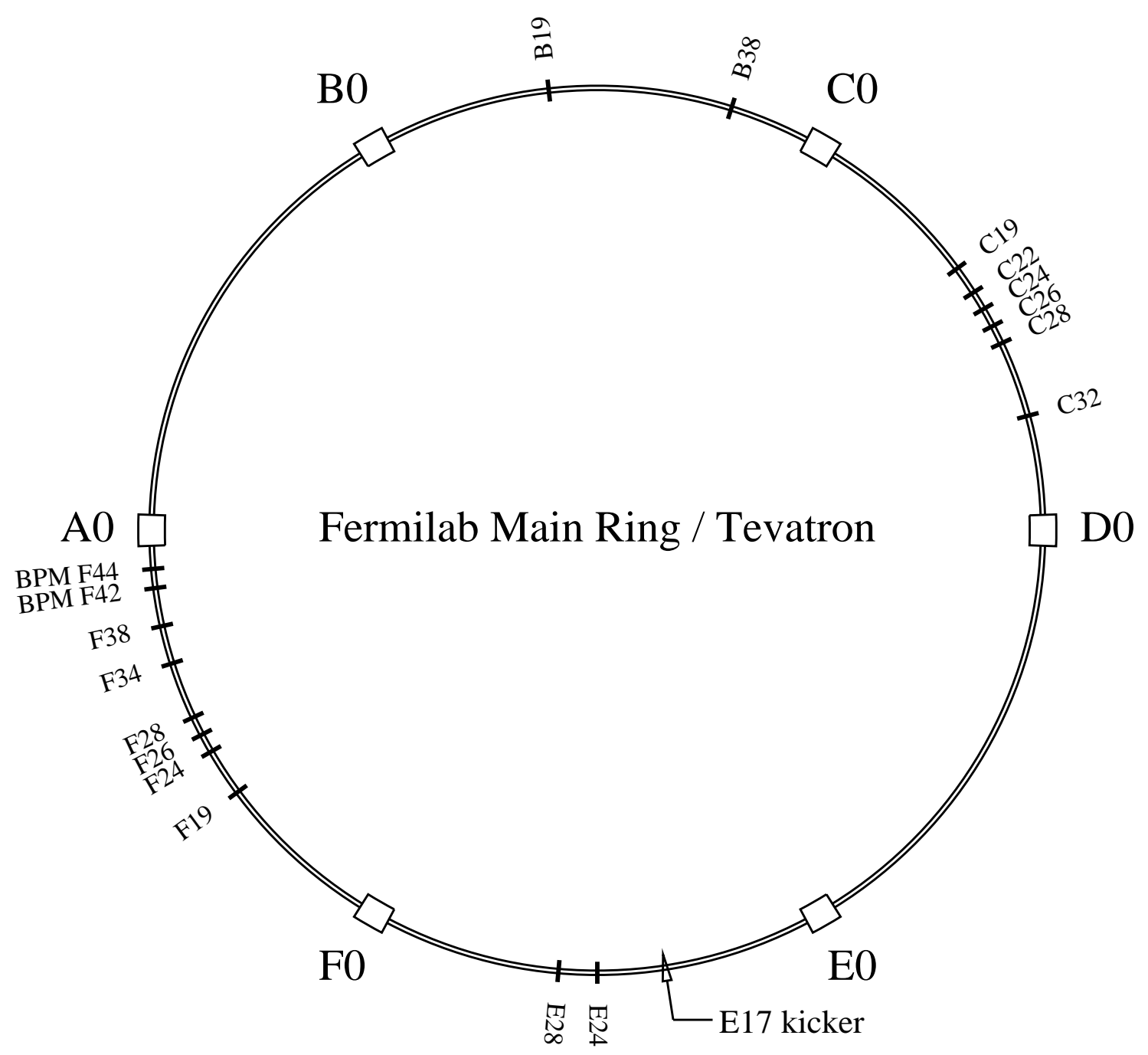

Figure 5.1: The lattice used in the January 1991 run of experiment E778, showing locations of sextupoles, beam position monitors and the E17 horizontal kicker. 
To investigate tune modulation effects, the tune modulation parameters must be varied over wide ranges and controlled with a high degree of accuracy. Because the synchrotron frequency of off-momentum particles cannot be widely varied due to RF system considerations, using tune modulation produced by such oscillations is impractical. Instead a quadrupole or set of quadrupoles with low inductance can be powered sinusoidally, creating tune modulation directly. A set of quadrupoles exist in the Tevatron that are powered by a $720 \mathrm{~Hz}$ digital waveform; they are primarily used for fine adjustments during slow extraction for fixed target operation. These quadrupoles are described in more detail in Section 2.1 of this chapter.

There are other less unusual but no less important requirements for control of the accelerator lattice in this experiment. Linear coupling must be minimized, since this causes the coherent transverse oscillation produced by the kicker to couple into the unkicked plane. Chromaticity must be reduced to as small a value as possible, typically one or two units, so tune modulation induced by bunched beam synchrotron oscillations does not interfere with the controlled tune modulation of the modulated quadrupoles. These are both normal operational procedures in the Tevatron and can be easily be performed while on experimental shifts.

\subsubsection{Data Acquisition Requirements}

The data acquisition system used for E778 used is based on Sun CPU architectures, dual crates (both VME and Camac) and twin LeCroy $68105 \mathrm{MHz} 12-$ bit transient digitizers. It is diagrammed in Figure (5.2). The data acquisition system requires three inputs from the central accelerator control system: a turnby-turn clock used to gate the turn-by-turn digitizers (which must be appropriately synchronized to the kicked bunch), a trigger to start the digitizing process, and the BPM signals themselves. The general configuration as a simple portable unix-based data acquisition system was originally implemented as MIRABILE at 


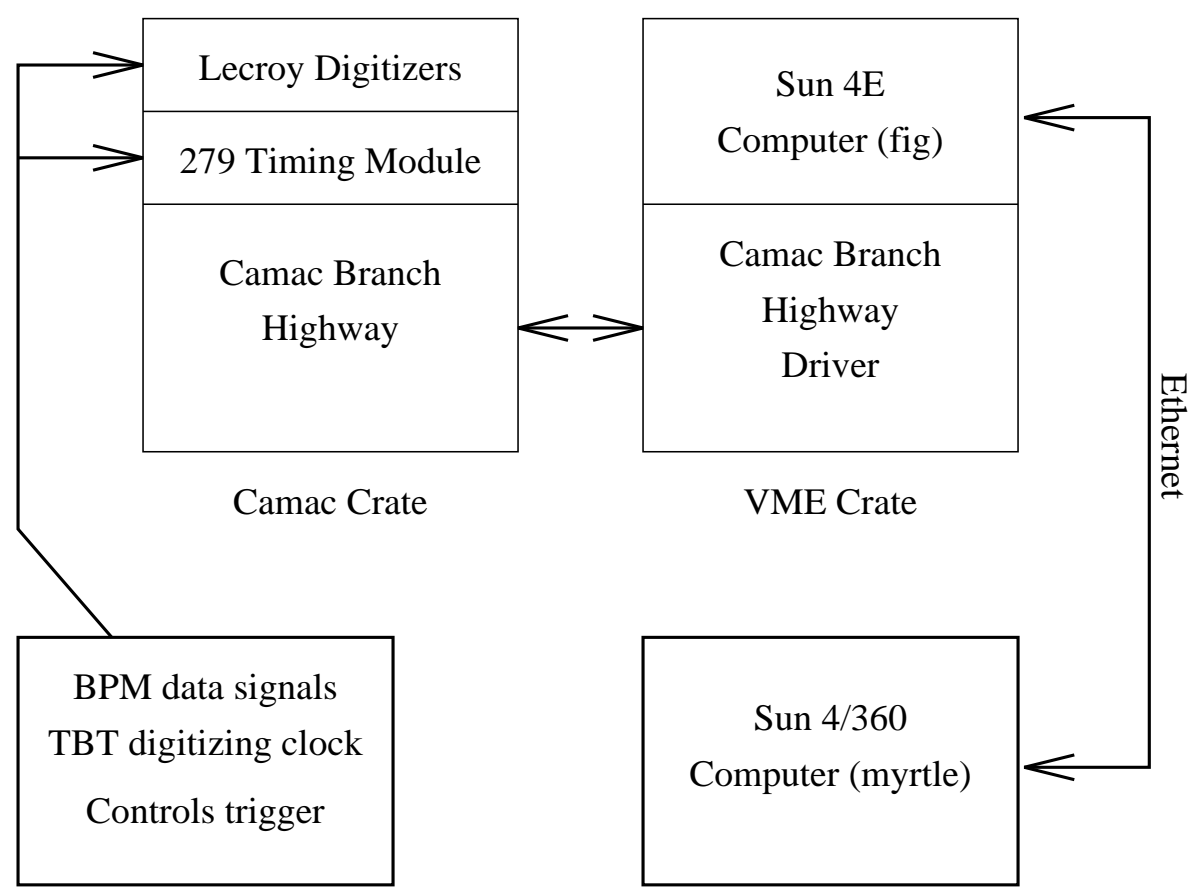

Figure 5.2: A block diagram of the E778 data acquisition system, showing both VME and Camac crates, general networking, and input signals.

Fermilab and the Cornell Electron Storage Ring (CESR) (Peggs, Saltmarsh and Talman 1987).

The turn-by-turn clock was supplied by the RF clock of the Tevatron divided by 1113 , since there are $1113 \mathrm{RF}$ cycles in one revolution of the machine when the $53 \mathrm{MHz}$ bunching RF is on. A programmable delay to synchronize this clock with the kicked bunch was created by a Fermilab Camac 279 module which delays in increments of 7 RF cycles. The acquisition trigger was supplied by a programmable 345 card synchronized to a timing event in the Tevatron timeline, allowing data acquisition either to begin on the order of thousands of turns before the E17 kicker was fired, allowing observation of decoherence for detuning measurements, or hundreds of thousands of turns after the kick, when transients had damped and 
the only coherent oscillation still remaining was the persistent signal.

The beam position monitor signals were acquired from the voltage difference of individual plates of parallel plate horizontal BPMs at the F42 and F44 Tevatron

locations. Front-end hardware included a peak-detector and a pulse stretcher with a decay time on the order of several microseconds. This setup gives output signals which are appropriate for digitization by the Lecroy 6810 digitizers. For the frequency-domain data analysis presented later in this chapter, note that full phase space reconstruction is not necessary and so only one digitized BPM signal, from F42, was used in this analysis.

A significant amount of software development also was needed for this project. The entire set of data acquisition system software was developed in $\mathrm{C}$ and $\mathrm{C}++$ using the tools of ISTK (the Integrated Scientific Toolkit) developed by Chris Saltmarsh, Vern Paxson and others (Lutz 1991). The data transfer protocol of ISTK is SDS, the self-describing data standard which allows storage of all turnby-turn data in simple processor-portable form. ISTK also includes a sequencing language and executive, Glish, which allows the control of many dependent tasks, or agents. For E778 there were several of these agents, including Camel (the Lecroy 6810 control program), Harc (the data archiver), Soc (the main experiment user interface) and Clod (the delay controller for the Camac 279 delay module). The Glistk graphics library, developed on top of the native $\mathrm{C}++$ window management system InterViews, was used to write graphical user interfaces for all of these programs. A sample user interface for Camel is shown in Figure (5.3) with typical digitizer settings for the January 1991 E778 run.

\subsection{LOCAL PREPARATION FOR THE EXPERIMENT}

\subsubsection{Magnet and Kicker Calibration}




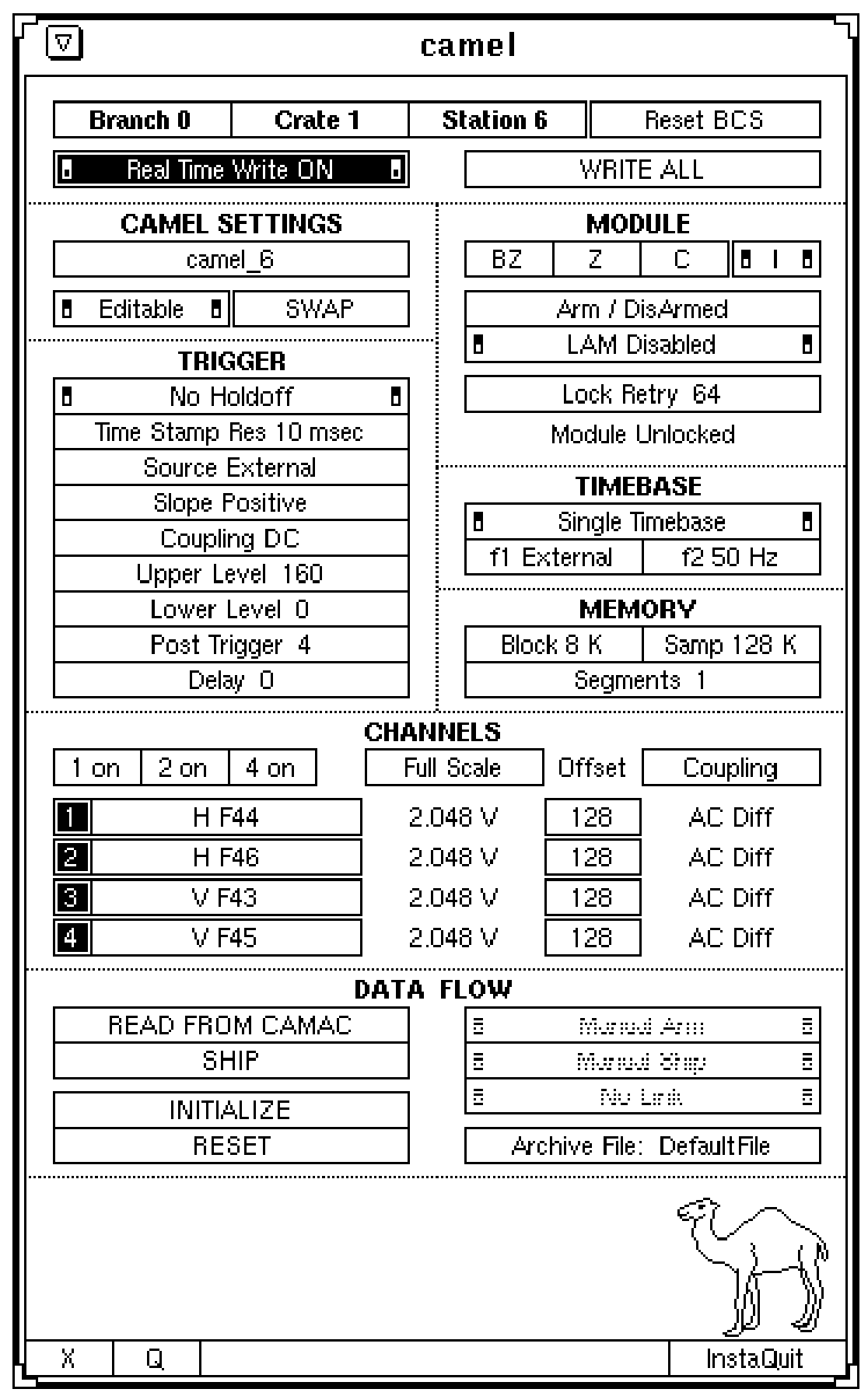

Figure 5.3: Camel, the graphic user-interface and control program for a Lecroy model 6810 digital waveform recorder. Settings are typical for those used in the January 1991 run of experiment E778. 


\begin{tabular}{|c|c|c|c|c|c|c|c|c|}
\hline \multirow{2}{*}{ Configuration } & \multicolumn{7}{|c|}{ Sextupoles } \\
\cline { 2 - 9 } & E24 & F19 & F24 & F34 & B19 & C19 & C22 & C24 \\
& E28 & F26 & F28 & F38 & B38 & C26 & C28 & C32 \\
\hline \hline $91 \_0$ & $+/-$ & $+/-$ & $-/+$ & $+/-$ & 0 & $+/-$ & $+/-$ & $+/-$ \\
$91 \_1$ & 0 & $+/-$ & $+/-$ & $-/+$ & 0 & $+/-$ & $+/-$ & $-/+$ \\
$91 \_2$ & $+/-$ & $+/-$ & $+/-$ & $-/+$ & 0 & $+/-$ & $+/-$ & $-/+$ \\
\hline
\end{tabular}

Table 5.1: Sextupole polarities and locations for 1991 E778 configurations. The first sign indicates the polarity of the first listed sextupole in each column. All sextupoles used in the actual tune modulation experiment were driven at a current of 30 amperes.

Knowledge of the sextupole strengths and their variation with current is crucial. Experimental results cannot reasonably be expected to match those of particle tracking if the strengths and locations of the strong nonlinearities are not known accurately and if other sources of nonlinearity are not accounted for.

Calibration measurements performed before the sextupoles were installed measured a magnetic field of $B_{s}=0.148 \mathrm{~T}$ at $r=1$ inch from the magnet center; the sextupoles also have length $L_{\text {sex }}=.732 \mathrm{~m}$. Using the magnetic rigidity $|B \rho|=500$ $\mathrm{T}-\mathrm{m}$ for the Tevatron at its injection energy of $150 \mathrm{GeV}$ where the experiment was performed gives the normalized sextupole strength of the sextupoles used at a current of 50 amperes:

$$
\tilde{b}_{2}=\frac{B_{0} L}{|B \rho|} b_{2}=\frac{B_{s} L}{|B \rho| r^{2}}=0.336 \mathrm{~m}^{-2}
$$

During the actual run the sextupole currents used were 30 Amps, with a corresponding normalized sextupole strength of $\tilde{b}_{2}=0.201 \mathrm{~m}^{-2}$.

The sextupoles are also ganged together on 9 separate buses, paired with opposite polarities as listed in Table (5.1) with the exception of the B19 and B38 sextupoles which are individually powered. The configuration 91_0 listed in this table is the nominal operational configuration, and this configuration and 91_1 were those investigated in the actual tune modulation experiment. A decision 
was made to turn off the B19 and B38 sextupoles to limit the number of configurations investigated in preliminary tracking.

The QXR quadrupoles used for tune modulation were also calibrated before the experimental run, and again during the initial startup shifts. These quadrupoles are driven by a digital waveform generated with a clock rate of $720 \mathrm{~Hz}$, so they can modulate the tune at frequencies up to $360 \mathrm{~Hz}$ or $Q_{M}=7.55 \cdot 10^{-3}$. Calibration was performed at DC current to measure the variation in tune versus quadrupole current, and showed the expected linear response in Figure (5.4), giving the tune changes

$$
\begin{gathered}
\Delta Q_{x}=4.1 \cdot 10^{-4} I_{Q}, \\
\Delta Q_{y}=-1.0 \cdot 10^{-4} I_{Q},
\end{gathered}
$$

where $I_{Q}$ is the quadrupole current in amperes. For the QXR system a current of 5 amperes was provided by a current supply for 1 volt setting on the control system, and the current range for this supply was 0 to 50 amperes. For a maximum modulation amplitude of 25 amperes or $q=1.02 \cdot 10^{-2}$, a constant current was of 25 amperes was superimposed on the sinusoidal modulation when the QXR system was used.

AC calibration of the QXR system is significantly more difficult, since tune measurements currently cannot be acquired more quickly than once or twice per second in the Tevatron. A calibration of the QXR quadrupoles was instead performed by setting the voltage on the voltage source to a certain value with a sinusoidal modulation with an amplitude of 5 amperes and observing the readback of the actual current supplied to the quadrupoles. This procedure allows measurement of the inductive decay of quadrupole response with rising frequency, showing a hyperbolic rolloff beginning near $100 \mathrm{~Hz}$ as in Figure (5.5). This measurement was performed during Tevatron downtime without making physical measurements of tune changes on the actual beam. 


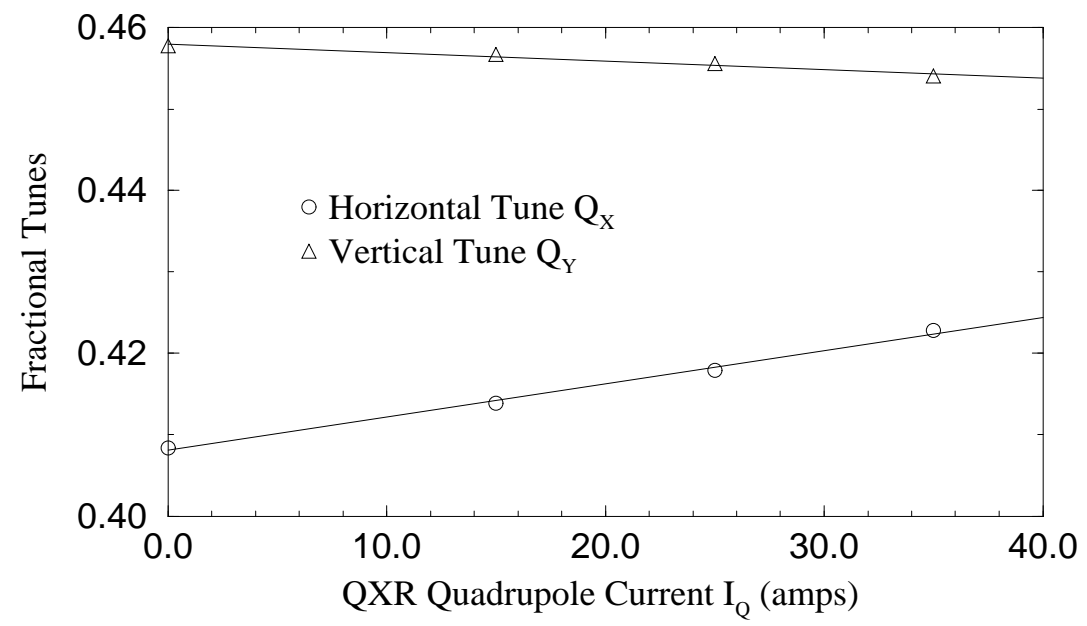

Figure 5.4: DC QXR quadrupole calibration, showing horizontal and vertical tune versus quadrupole current $I_{Q}$. Linear fits give $\Delta Q_{x}=$ $4.1 \cdot 10^{-4} I_{Q}$ and $\Delta Q_{y}=-1.0 \cdot 10^{-4} I_{Q}$.

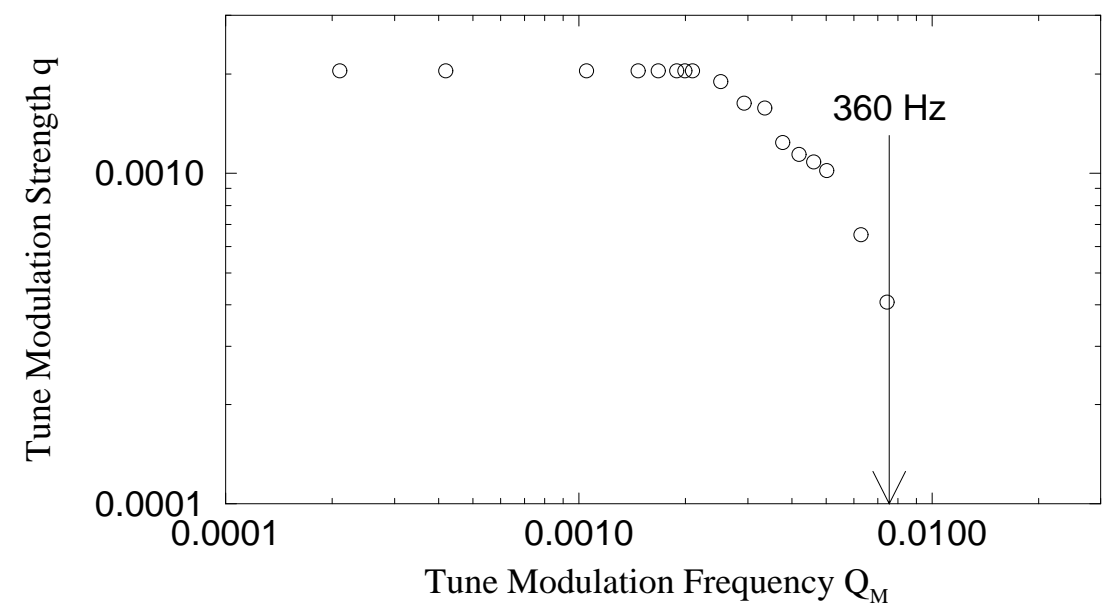

Figure 5.5: AC QXR quadrupole calibration, performed at 5 amps excitation current corresponding to tune modulation strength $q=2 \cdot 10^{-3}$ Response rolloff begins at $100 \mathrm{~Hz}$, or a tune modulation frequency of approximately $Q_{M} \approx 2 \cdot 10^{-3}$. 
There is some concern about the effects of skin depth and penetration into the stainless steel beam pipe of the Tevatron by the field of the QXR quadrupoles. The conductivity of this material is approximately $\sigma=4 \cdot 10^{7}(\mathrm{ohm} \mathrm{m})^{-1}$, giving a skin depth of approximately $4 \mathrm{~mm}$ at frequencies of $350 \mathrm{~Hz}$. This is several times the actual thickness of the beam pipe in the Tevatron, approximately $1.5 \mathrm{~mm}$, and so for all frequencies involved in the chirp there should be negligible damping of the quadrupole modulation amplitude by conductive effects of the beam pipe.

\subsubsection{Preparatory Tracking and Simulations}

Once the sextupole strength scaling versus excitation current is known, tracking can be performed to find the optimal conditions for beam capture in a resonance island. The transverse phase space should contain large resonance islands, similar to those pictured in Figure (3.1). These resonance islands should not be severely distorted by the presence of the $3 Q_{x}$ resonance, which is driven to first order by the sextupoles - previous E778 runs have measured such distortions (Chao et. al. 1987b, Merminga and Ng 1992). The resonance islands should also have fixed point phases positioned in such a manner than a stable fixed point lies in the $x^{\prime}$ direction of the phase space at the E17 kicker; this maximizes the amount of beam captured in the resonance island and the persistent thus produced. And finally, tracking should predict island tunes that fall within the experimentally accessible range for the tune modulation experiment.

Beta functions and phase positions of the sextupoles and E17 kicker were found using the most recent version of the Tevatron lattice and the accelerator design code MAD 8.1. A lattice was designed in Evol using only the sextupoles and linear phase advances, and the phase space at the kicker for a variety of configurations was produced. Of several configurations investigated, the configurations 91 1 and 91_2 satisfied the above criteria, as well as the nominal configuration 91_0. During 


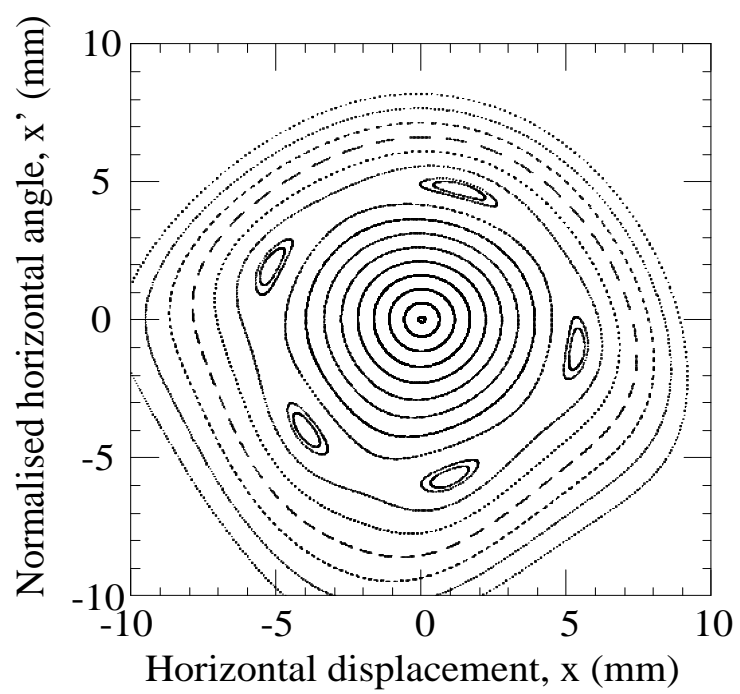

Figure 5.6: Transverse phase space for the 91_0 sextupole configuration at the E17 kicker. Tracking was performed with Evol with base tunes $\left(Q_{x}, Q_{y}\right)=(20.394,20.460)$.

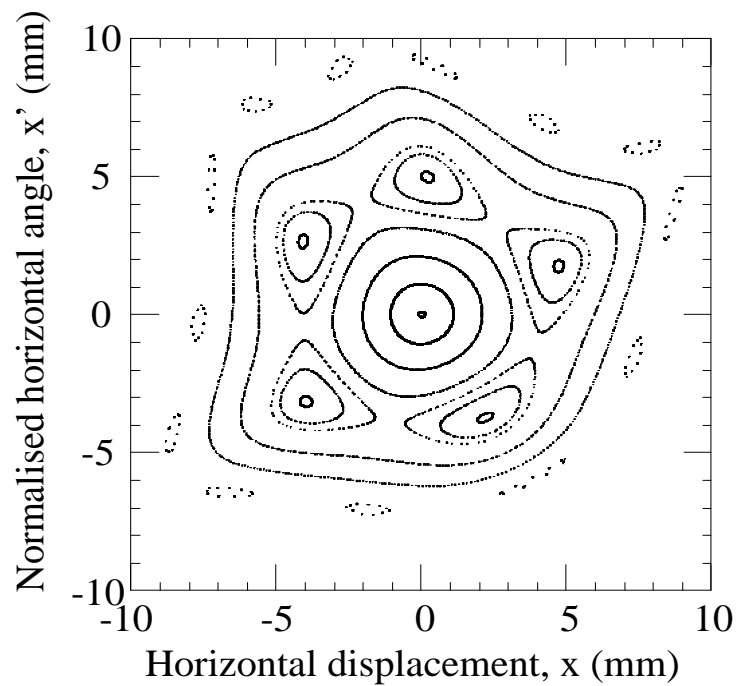

Figure 5.7: Transverse phase space for the 91_1 sextupole configuration at the E17 kicker. Tracking was performed with Evol with base tunes $\left(Q_{x}, Q_{y}\right)=(20.406,20.460)$. 
the experimental run only configuration $91 \_1$ and the nominal configuration 91_0 were investigated.

Figures (5.4) and (5.5) show the phase spaces produced by the 91_0 and 91_1 sextupole configurations, respectively. Each has a separate variation of tune with amplitude, necessitating different base tunes for these phase space diagrams in order to detune into the resonance and display resonance islands, and both show resonance islands that are positioned properly without severe distortion from the presence of the third-integer resonance. The island tunes for each of these sex-

tupole setups are $335 \mathrm{~Hz}\left(Q_{\mathrm{I}}=7 \cdot 10^{-3}\right)$ and $700 \mathrm{~Hz}\left(Q_{\mathrm{I}}=1.9 \cdot 10^{-2}\right)$ respectively, indicating that the resonant region should be barely accessible for lattice 91_0, but not for lattice $91_{-} 1$. There are also small $13 Q_{x}$ resonance islands evident in the phase space of the simulated 91_1 lattice, indicating that capture on resonances other than the $5 Q_{x}$ is feasible in these configurations.

\subsection{THE EXPERIMENTAL RUN}

The experimental run of this section of E778 took place during two weekends in January, 1991, lasting a total of fourteen eight-hour shifts. Of those shifts, nearly a third each were dedicated to setup, the diffusion experiment and the persistent signal and tune modulation experiment.

Shifts for the first weekend were dedicated to debugging and testing the data acquisition system, calibrating and testing tune modulation quadrupoles, and scanning various kicker voltages, base tunes and nonlinearity configurations to find persistent signals. For this section of the weekend, the data acquisition system acquired 64 kiloturns of data, or approximately 1.4 seconds of data per datataking "shot". Persistent signals were located at $Q_{x}=0.375$ (the $8 Q_{x}$ resonance), $Q_{x}=0.400$ (the $5 Q_{x}$ resonance), and $Q_{x}=0.417$ (the $12 Q_{x}$ resonance). The E17 kicker kick amplitude was also calibrated versus applied kicker voltage in the 


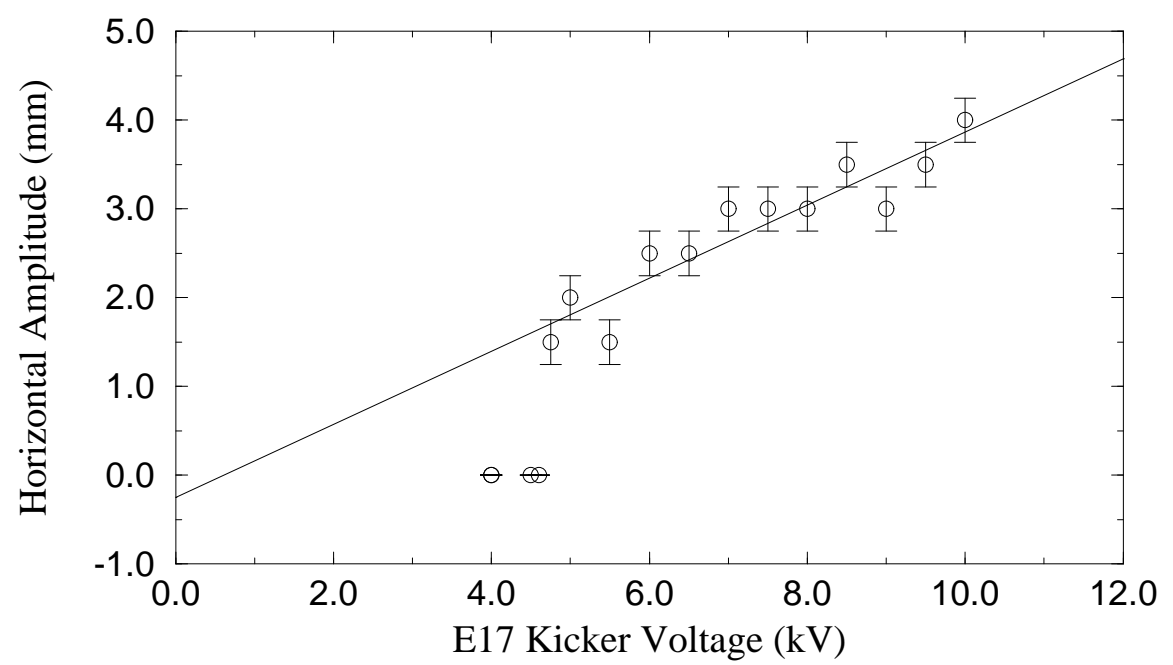

Figure 5.8: Calibration of the E17 kicker voltage versus kick amplitude. The linear fit slope is $0.41 \mathrm{~mm} / \mathrm{kV}$, and the kicker does not fire at voltages below 4.75 kilovolts.

early portion of the run; response was found to be approximately linear, with a $.41 \mathrm{~mm} / \mathrm{kV}$ slope. Samples of the turn-by-turn data produced online by the data acquisition system are shown in Figures (5.9) and (5.10), showing both a resonant persistent signal and gaussian decoherence without beam capture:

The event timeline for a typical 2 minute machine cycle or "shot" was as follows:

- Inject beam into Tevatron at $150 \mathrm{GeV}$, without acceleration, and coalesce to single bunch. (0-7 seconds)

- Reduce normalized beam emittance from $15 \pi \mathrm{mm}-\operatorname{mrad}$ to $3 \pi \mathrm{mm}-$ mrad with scraper at Tevatron lattice location D17 (10-30 seconds)

- Ramp up sextupole currents and trigger E17 kicker. (35-45 seconds)

- Wait approximately 10 seconds for transients from the transverse kick to settle. (45-56 seconds)

- Trigger data acquisition system and tune modulation quadrupoles. (56-58 seconds)

- Data transfer, sextupole ramp down and preparation for the next 


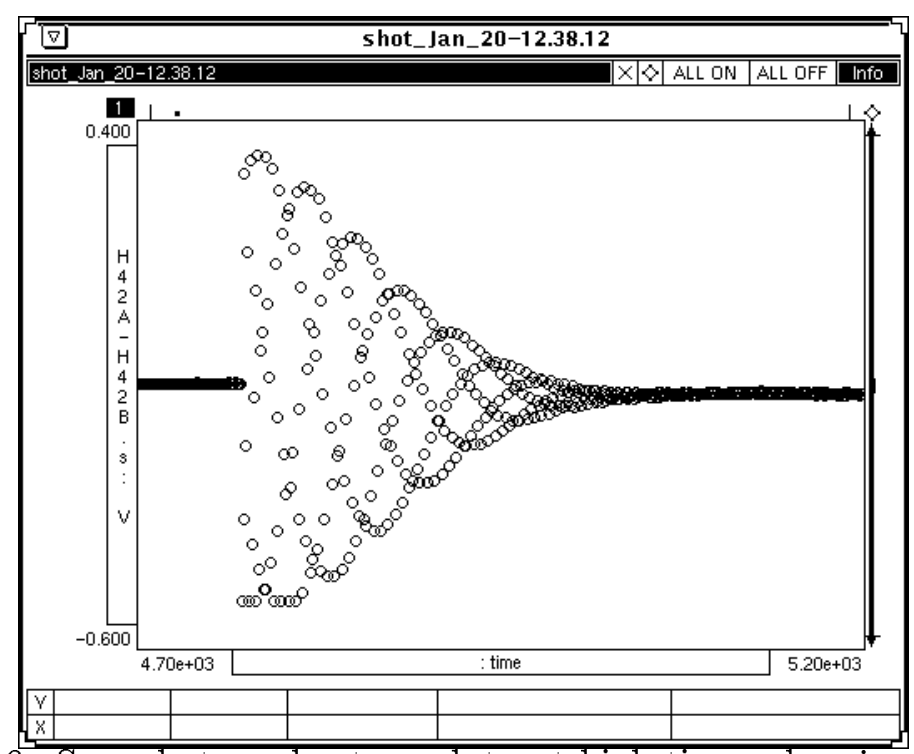

Figure 5.9: Sample turn-by-turn data at kick time, showing the kick and gaussian decoherence. Kicker voltage is $11 \mathrm{kV}$, and the lattice is 91_0; graphics are produced by the kaspar graphics program.

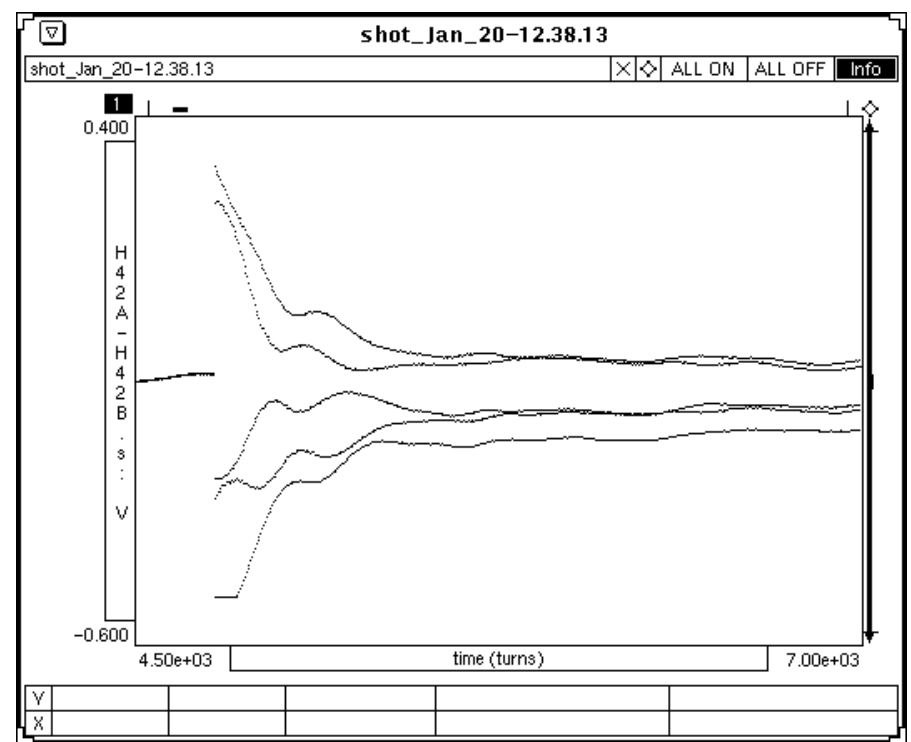

Figure 5.10: Sample turn-by-turn data at k1ck time, showing the kick and production of a $Q_{x}=20.40$ persistent signal. Kicker voltage and lattice are as in above figure. 


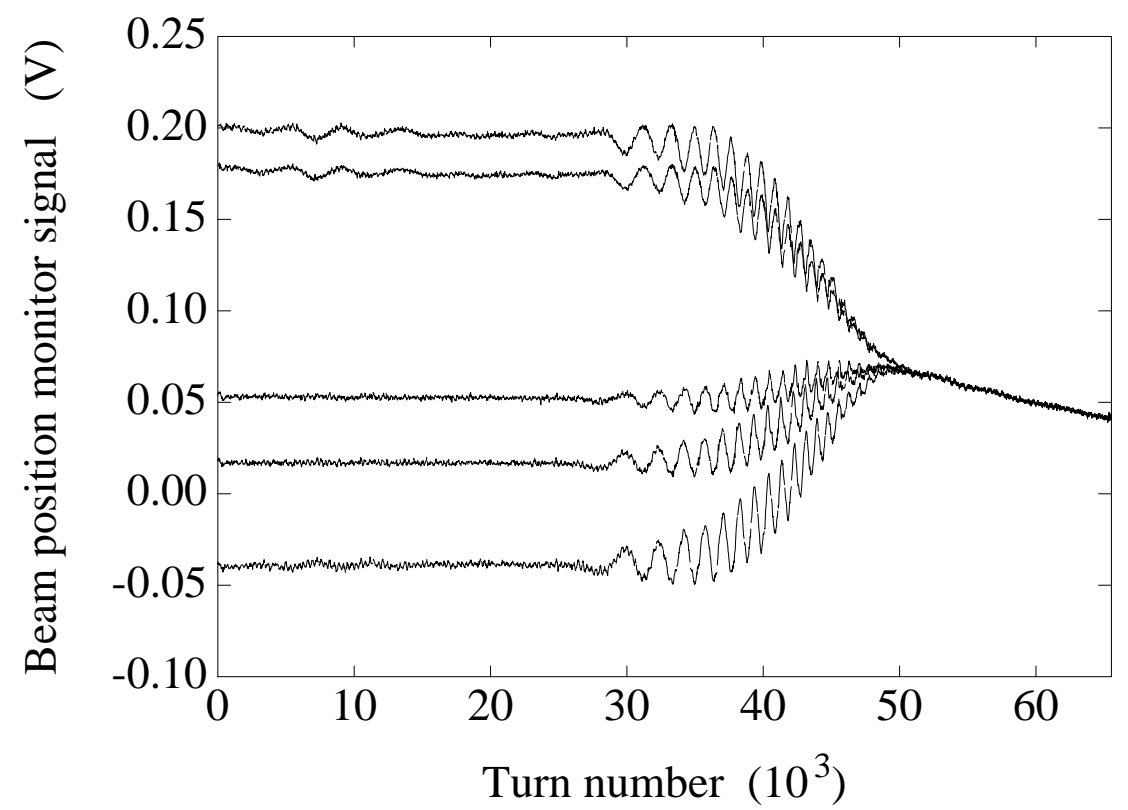

Figure 5.11: Turn-by-turn data for a strong persistent signal destroyed by chirped tune modulation. The tune modulation strength and frequency were ramped from $\left(q, Q_{M}\right)=(0,0)$ to $\left(1.0 \cdot 10^{-2}, 3.1 \cdot 10^{-3}\right)$. Lattice configuration is 91_0.

shot. $(60-120$ seconds)

Some unsystematic tune modulation data were also acquired in the first weekend for the nominal 91_0 lattice, starting approximately 9 kiloturns after the data acquisition system was activated and ramping for one second, or 47.7 kiloturns. An excellent example of the turn-by-turn data thus produced is shown in Figure (5.11) where the tune modulation parameters are chirped from $\left(q, Q_{M}\right)=(0,0)$ to $(1.0$. $\left.10^{-2}, 3.1 \cdot 10^{-3}\right)$. At approximately 28 kiloturns amplitude modulation of the islands becomes clearly visible, and the resonant response of the system destroys the persistent signal between 35 and 45 kiloturns.

On the second weekend systematic tune modulation scans were performed in order to make the search of the available tune modulation parameter space more efficient. For QXR voltages ranging roughly logarithmically from 0.1 volts to 5 


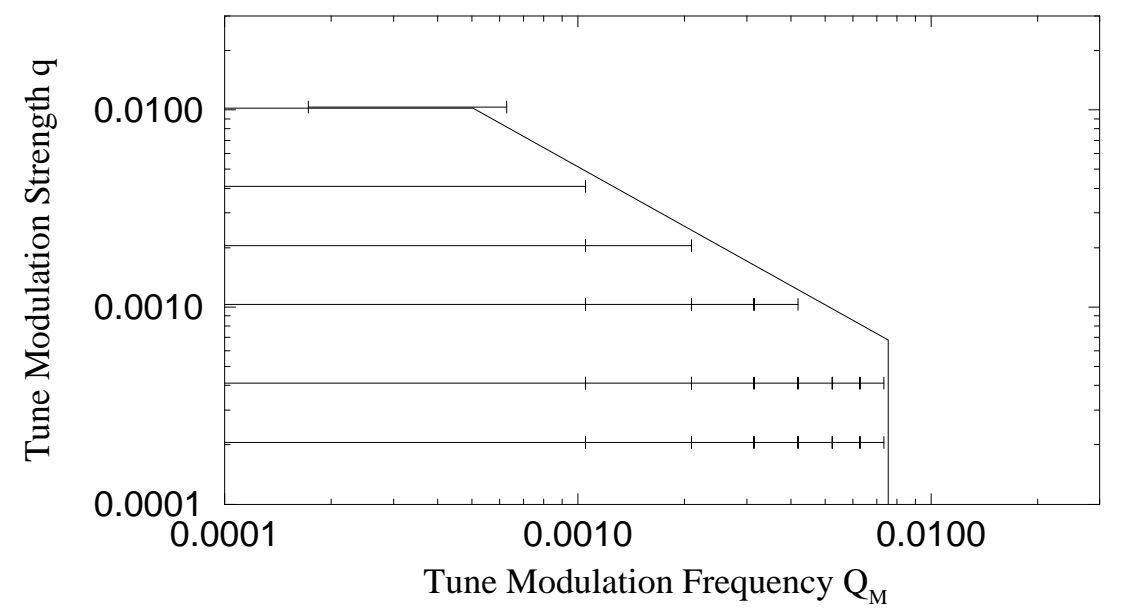

Figure 5.12: Distrubution of systematic tune modulation scans.

volts $\left(q=2 \cdot 10^{-4}\right.$ to $\left.10^{-2}\right)$, chirps were performed in $50 \mathrm{~Hz}$ segments within the region of linear quadrupole response. During this second weekend such scans were performed for two kick amplitudes $(5 \mathrm{kV}$ and $9 \mathrm{kV})$ with the 91 1 configuration, and two kick amplitudes $(7 \mathrm{kV}$ and $9 \mathrm{kV})$ in the $91 \_0$ configuration, all concentrating on the $5 Q_{x}$ resonance. Some individual chirps ranging from 0 to $350 \mathrm{~Hz}$ were also performed at small modulation amplitudes. Upgrades to the data acquisition system also allowed digitization of 128 kiloturns of data per shot, approximately 2.7 seconds of real beam time.

\subsection{DATA ANALYSIS AND RESULTS}

Approximately one gigabyte of turn-by-turn data were taken in the January 1991 run of E778. This included both turn-by-turn data taken at kick time away from a resonance to measure the variation of tune with amplitude by decoherence, and turn-by-turn data taken after initial transients had decayed and tune modulation was turned on. This section describes the analysis of both the detuning and tune modulation data. 


\subsubsection{Measuring Detuning}

Previous results from E778 and elsewhere have observed and successfully compared measurements of the decoherence of a gaussian beam kicked transversely into sheared nonresonant phase space with theory (Chao et. al. 1987b, Merminga 1989, ByrdThesis). For a gaussian beam of transverse size $\sigma_{x}$ kicked to produce a coherent oscillation of amplitude $X_{0} \gg \sigma_{x}$ as observed at a beta function of $\beta_{o b s}$, the decoherence of the observed centroid position is predicted to be approximately gaussian (Chao et. al. 1987a):

$$
x(t) \approx X_{0} \exp \left[-\frac{1}{2}\left(\frac{2 \pi \sigma_{x} \alpha X_{0} t}{\beta_{o b s}}\right)^{2}\right],
$$

where $\alpha$ is the one-dimensional Hamiltonian detuning from Equation (3.7).

The analysis program Tevex developed by Peggs, Chen and Merminga (Merminga 1989) was used to fit turn-by-turn data from two horizontal BPMs to the predicted gaussian decoherence. Tevex uses a five-parameter fit: the exponential term in the gaussian decoherence, values for the closed orbit offsets (or digitizer voltage offset) at both BPMs, the phase advance between the BPMs and the ratio of the horizontal beta functions at the BPMs. Tevex also returns the horizontal tune and the horizontal smear. Two approaches can be taken to measure the detuning parameter $\alpha$ : if the beam size and the beta functions at the BPMs are accurately known, the fit of the gaussian decoherence exponent by Tevex gives the detuning. However, if these are not known but the base tune setting of the machine is, then one can calculate from the tune returned by Tevex how much the tune has shifted for a given kick and thus the detuning. This second procedure was the procedure followed here, though the former method returns similar detuning after substitution of a typical beam size and beta function.

Figures (5.13) and (5.14) display a comparison of the detuning data for both nonlinear configurations examined to tracking with Evol through these configu- 


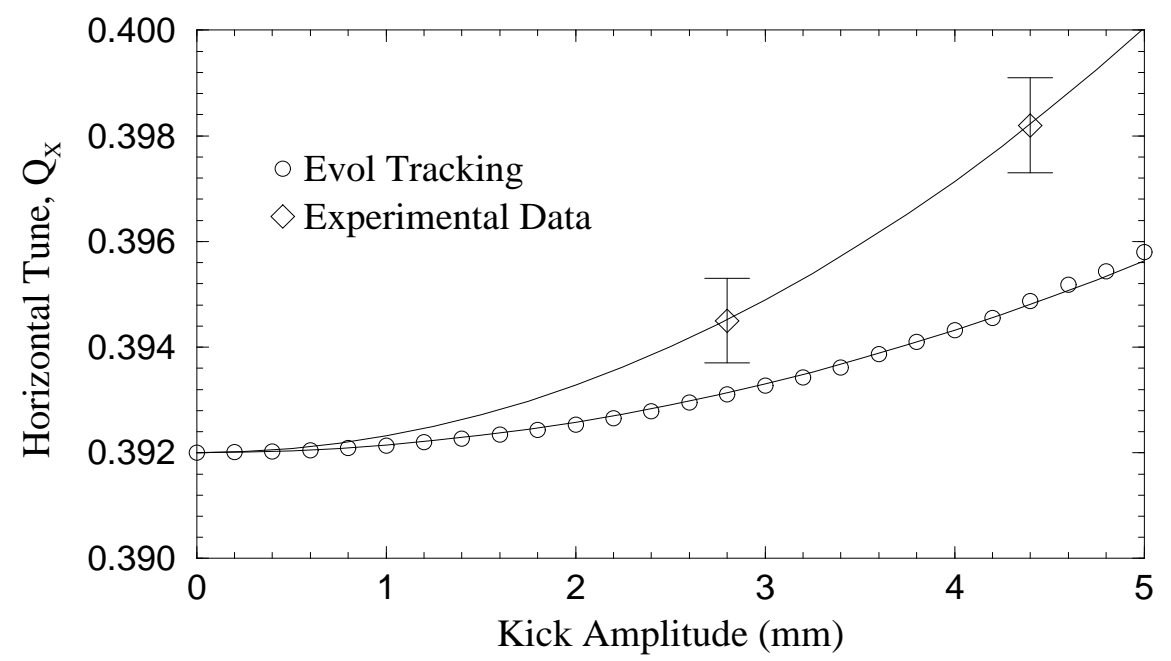

Figure 5.13: Comparison of Evol tracking to detuning data for the 91_0 sextupole configuration.

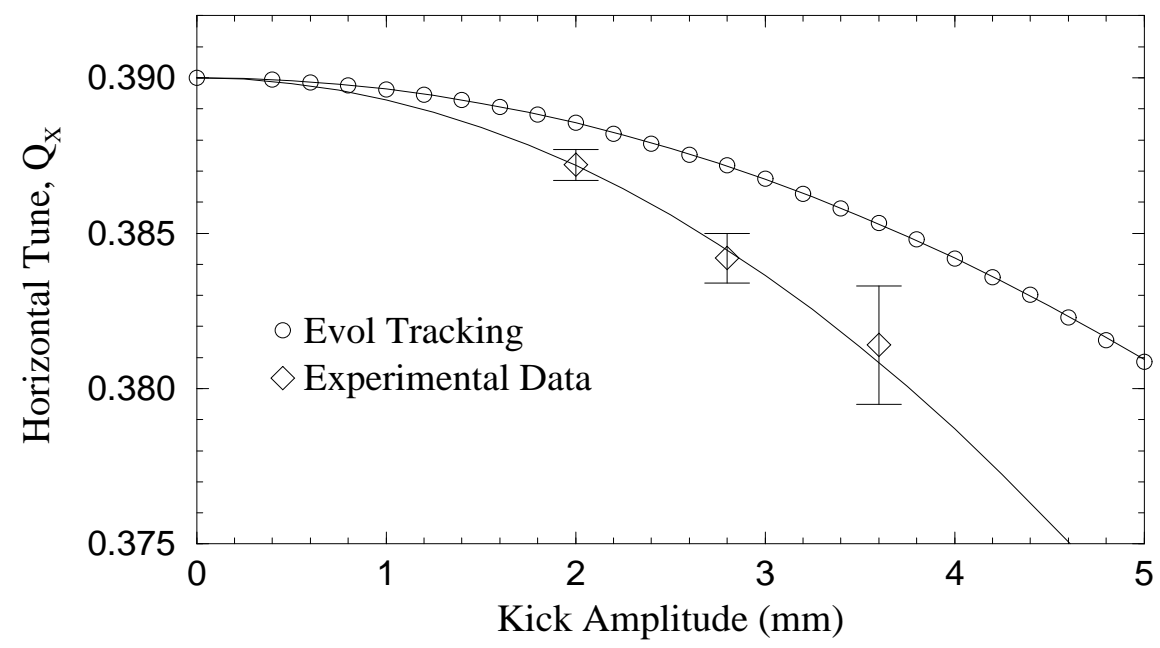

Figure 5.14: Comparison of Evol tracking to detuning data for the 91_1 sextupole configuration. 
rations, with lines displayed as fits to each set of data. The evident discrepancy between tracking and experiment can be due to several different factors. The base tune setting of the Tevatron was noted during the course of the run to be rather inaccurate, varying by as much as 0.005 units from set values. Allowing this base tune of the experimental measurement to vary by this amount provides a better fit of the detuning. The sextupole strengths used in tracking may also differ by those experimentally used; since the detuning is driven to second order in sextupole strength, a difference by a factor of 1.4 between simulated and experimental sextupole strengths is sufficient to reconcile the detuning as shown in these figures.

\subsubsection{Frequency Domain Behavior}

Because the boundaries between regions in the tune modulation stability plane $\left(q, Q_{M}\right)$ scale with the island tune, $Q_{\mathrm{I}}$, it is logical to expect the frequency response the of beam to give information about the rate at which the phase-locked portion of resonance island is shrinking in response to chirped tune modulation. A fast Fourier transform (FFT) applied to a segment of unmodulated persistent signal turn-by-turn data displays an extremely sharp peak at exactly the resonant tune due to the highly coherent nature of the island-to-island motion that occurs for trapped particles. As particles are lost from this coherent signal due to tune modulation, the amplitude of this peak in the FFT spectrum decays with a rate that indicates how quickly the stable portion of the resonance island is shrinking.

For a 128 kiloturn data set acquired during the second weekend, the data was partitioned into overlapping 8192 turn segments, and FFTs were performed. This gives a frequency resolution in the resulting power distribution of approximately $10^{-4}$ for each FFT. The amplitude of these FFTs were then plotted versus time.

The three-dimensional plots of FFT amplitude versus frequency within the FFT 
and turn number are displayed in Figure (5.15) through (5.22), for the configuration 91_0, a kicker voltage of $9 \mathrm{kV}$, the smallest modulation amplitude $q=2 \cdot 10^{-4}$ and both $50 \mathrm{~Hz}$ and $350 \mathrm{~Hz}$ frequency chirps. These diagrams all have a large spike exactly at the resonant frequency, 0.4 , which corresponds to the persistent signal - as the modulation detraps particles from the resonance island, this persistent signal decays. Progressing through the $50 \mathrm{~Hz}$ chirps, the persistent signal lifetime under modulation decreases steadily until the chirp from $300 \mathrm{~Hz}$ to 350 $\mathrm{Hz}$. The stability of this scan indicates that the island frequency has been passed by the modulation frequency, thus implying that the island tune to be somewhere between $250 \mathrm{~Hz}$ and $300 \mathrm{~Hz}$. For both configurations and sets of kicks examined, these are the only such data to exhibit behavior that indicates stability above the island frequency. We therefore discuss in the following how the island tune can be found more accurately from this data.

A convenient way to view this decay is by examining the instantaneous decay rate of the persistent signal from FFT to FFT, or as time (and the modulation frequency) increases. Assume that this decay is exponential and of the form

$$
A_{\mathrm{FFT}}(t)=A_{0} e^{-\gamma t}
$$

where $A_{0}$ is the initial FFT amplitude and $\gamma$ is the instantaneous persistent signal decay rate expressed in inverse turns. This gives a formula for the decay rate between any two times $t_{1}$ and $t_{2}$ :

$$
\gamma=\frac{\log A_{\mathrm{FFT}}(t 1)-\log A_{\mathrm{FFT}}(t 2)}{t_{2}-t_{1}}
$$

One particular advantage of this analysis is that it is independent of the initial Fourier amplitude $A_{0}$. If the BPM voltage varies linearly with centroid position, such an approach in the frequency (or tune) domain does not require scaling for variations in beam current from shot to shot or fraction of the beam captured. 


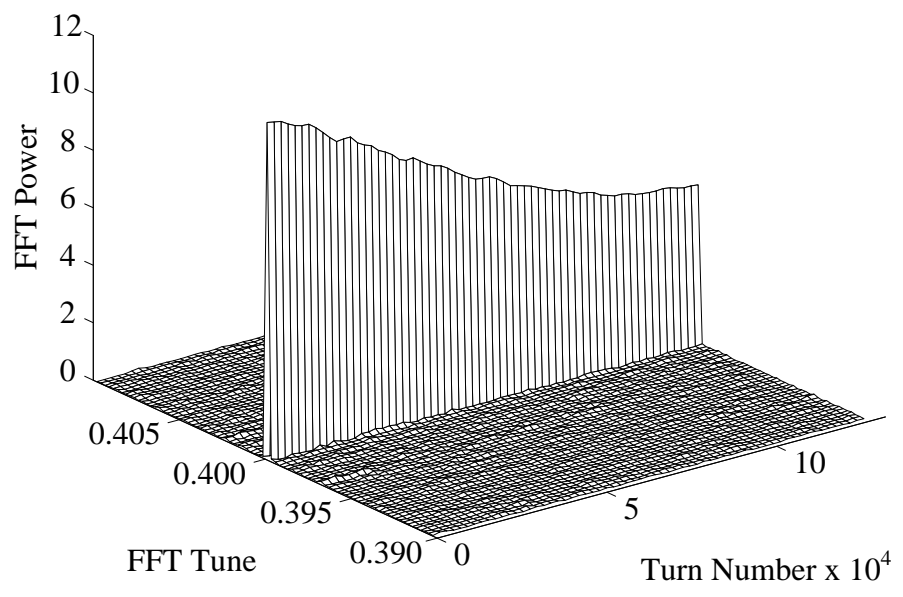

Figure 5.15: A segmented FFT for turn-by-turn tune modulation of the 91_0 sextupole configuration. Here $q=2 \cdot 10^{-4}$ and $Q_{M}$ is chirped from 0 to $50 \mathrm{~Hz}$. Overlapping FFTs are taken every 8192 turns, and the amplitude of these FFTs for the frequency range $(0.398,0.402)$ is displayed versus turn number, or modulation frequency during the chirp.

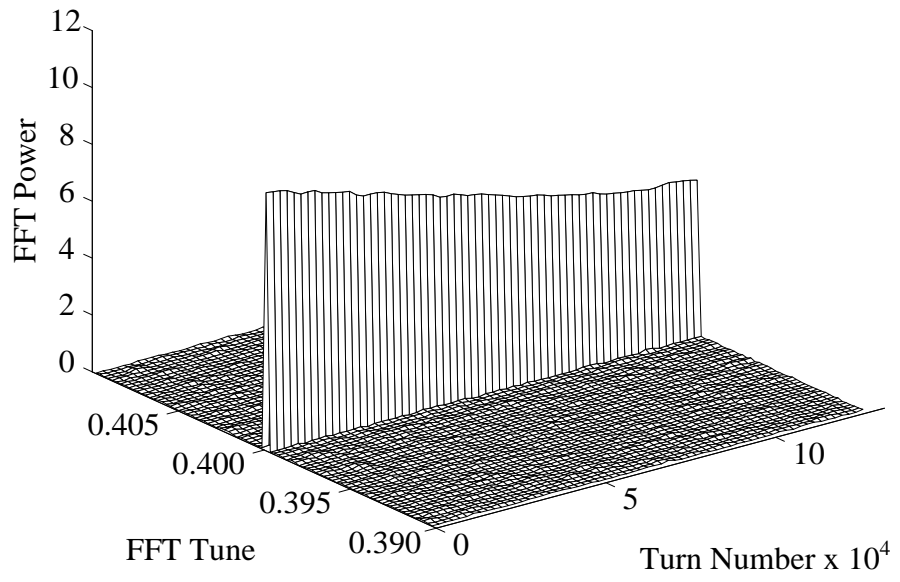

Figure 5.16: Same as Figure 5.15, with $Q_{M}$ chirped from 50 to $100 \mathrm{~Hz}$. 


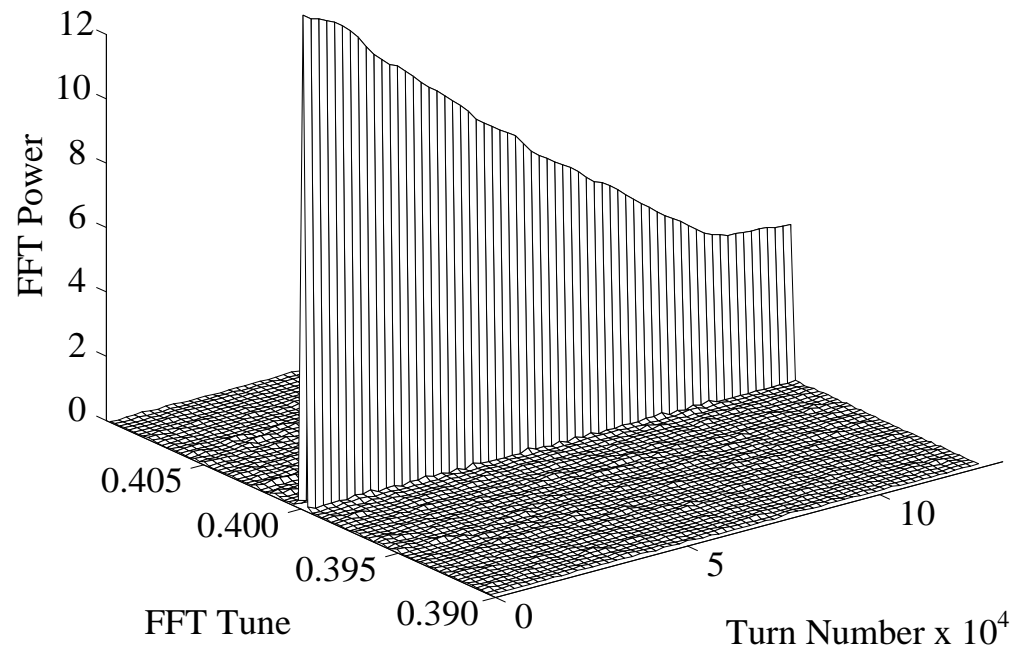

Figure 5.17: Same as Figure 5.15, with $Q_{M}$ chirped from 100 to $150 \mathrm{~Hz}$.

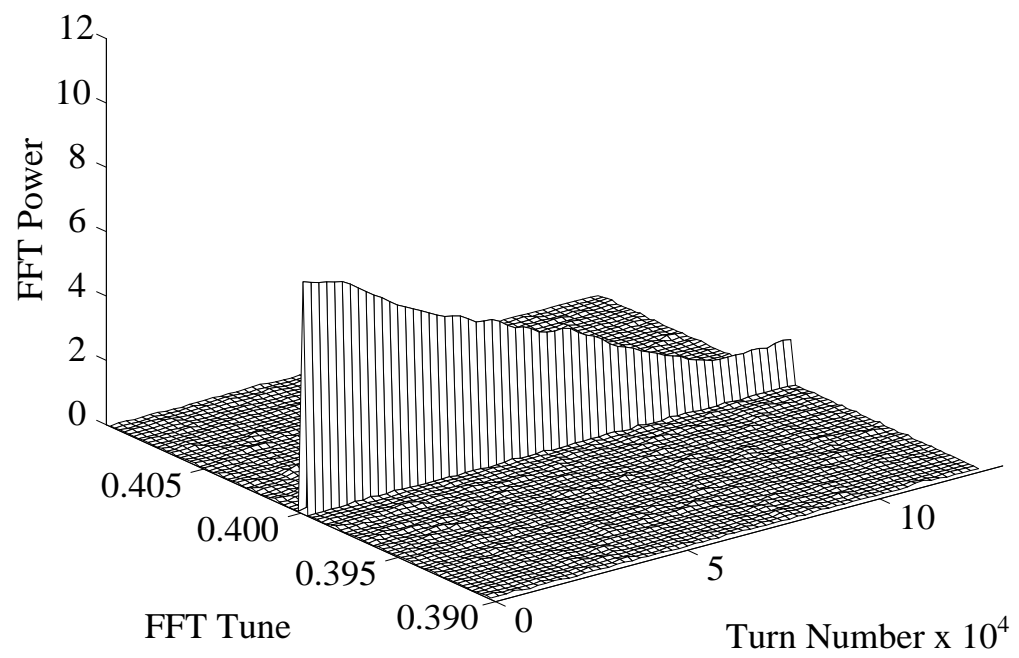

Figure 5.18: Same as Figure 5.15, with $Q_{M}$ chirped from 150 to $200 \mathrm{~Hz}$. 


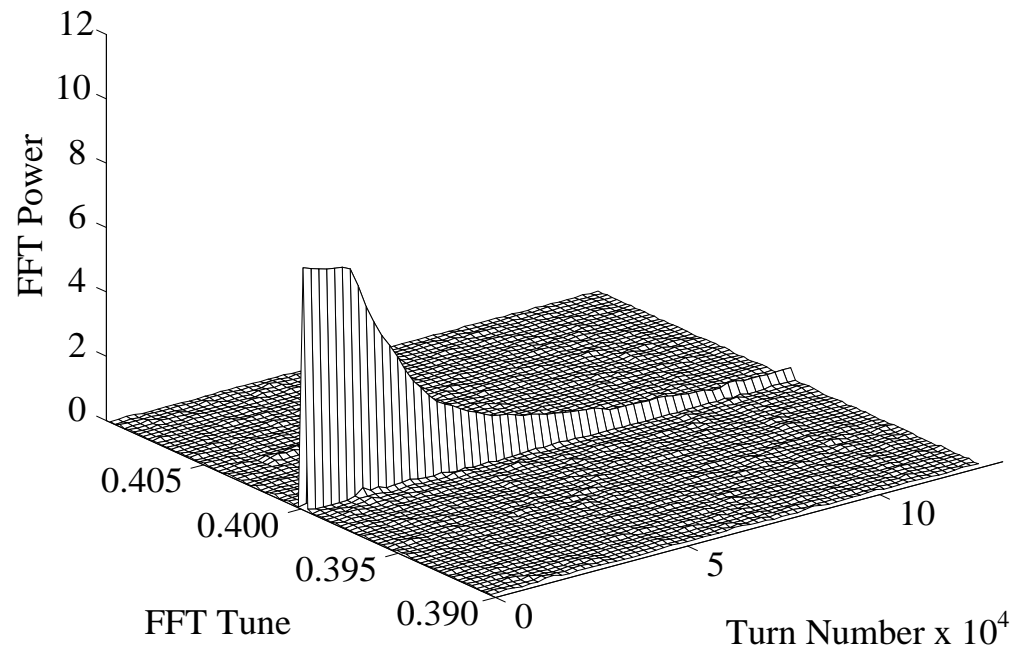

Figure 5.19: Same as Figure 5.15, with $Q_{M}$ chirped from 200 to $250 \mathrm{~Hz}$.

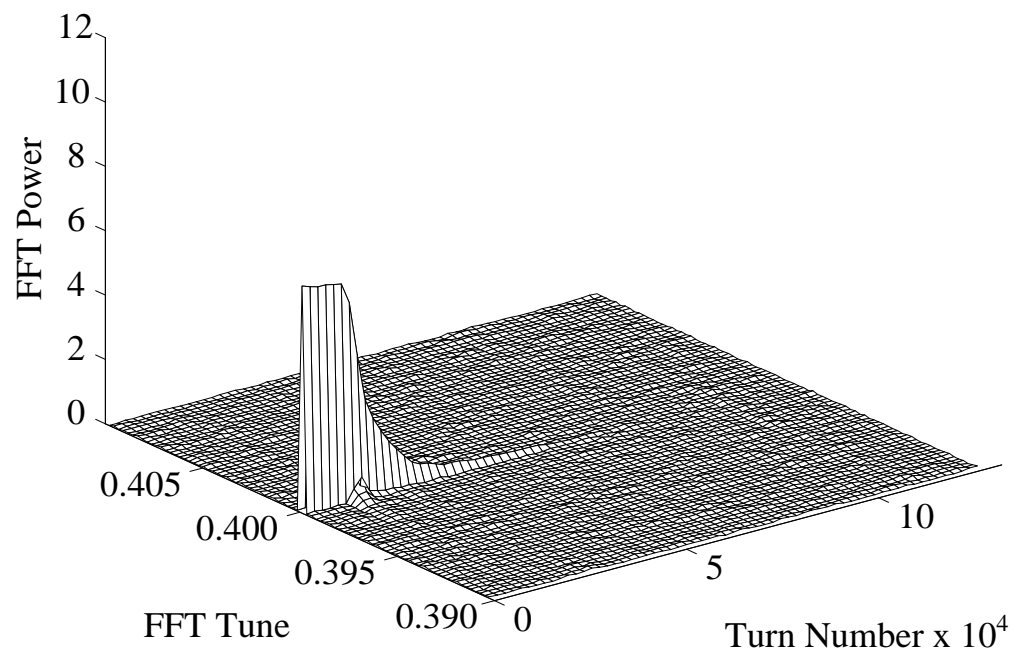

Figure 5.20: Same as Figure 5.15, with $Q_{M}$ chirped from 250 to $300 \mathrm{~Hz}$. 


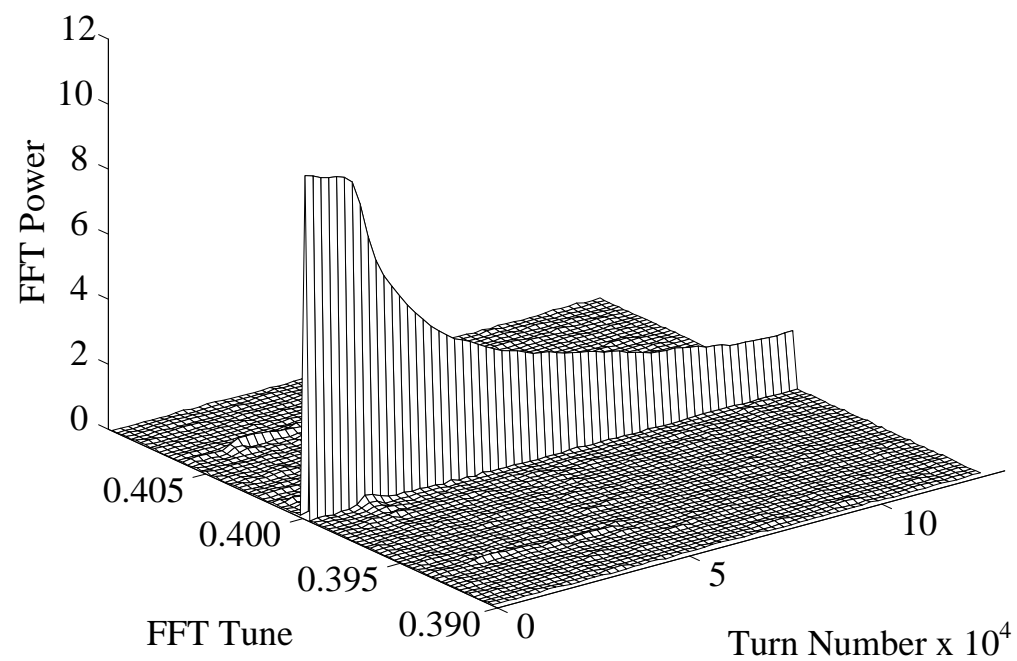

Figure 5.21: Same as Figure 5.15, with $Q_{M}$ chirped from 300 to $350 \mathrm{~Hz}$.

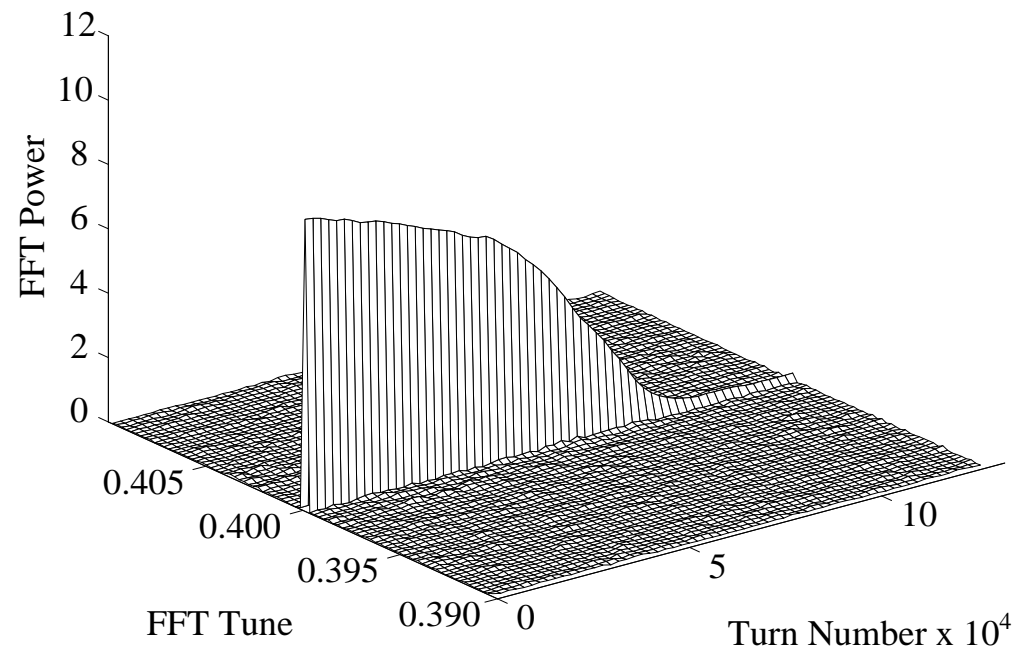

Figure 5.22: Same as Figure 5.15, with $Q_{M}$ chirped from 0 to $350 \mathrm{~Hz}$. 


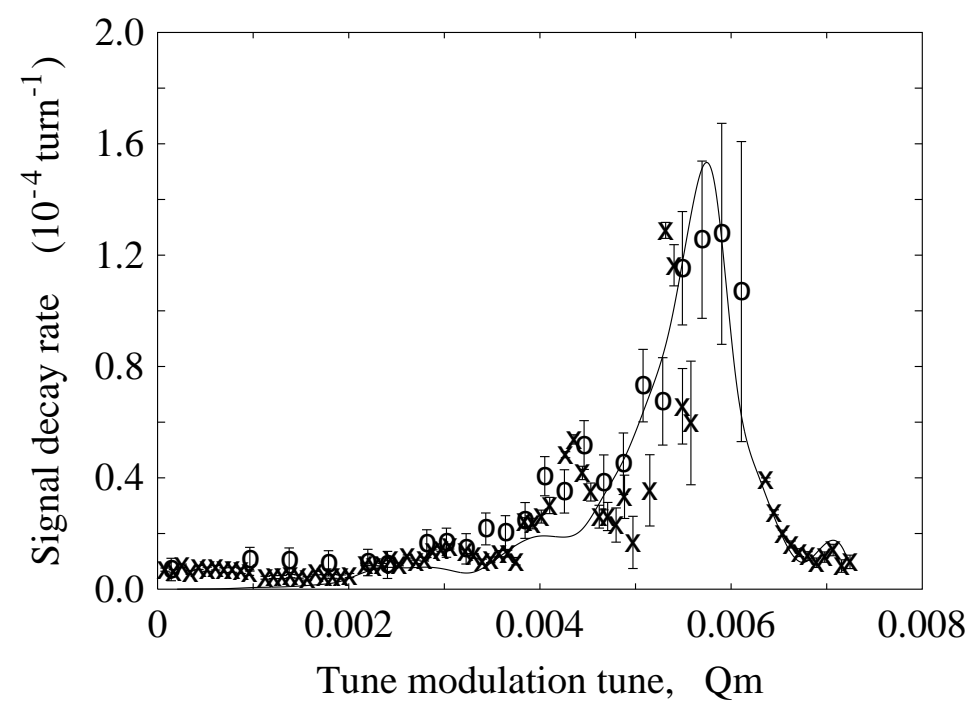

Figure 5.23: Persistent signal decay rate as a function of tune modulation frequency, $Q_{M}$ in configuration 91_0. The tune modulation amplitude is held constant at $q=2.04 \cdot 10^{-4}$. Circles represent experimental data from a single chirp from 0 to $350 \mathrm{~Hz}$, and crosses represent data from seven individual $50 \mathrm{~Hz}$ scans. The line is a cubic spline fit through simulation data from the program OdChirp, with $Q_{\mathrm{I}}=6.3 \cdot 10^{-3}$.

The decay rate data for this modulation amplitude are shown in Figure (5.23), with crosses representing $50 \mathrm{~Hz}$ frequency chirps and circles representing the 350 $\mathrm{Hz}$ chirp. Error bars are produced by scaling the remaining persistent signal to the initial persistent signal, since a smaller signal size gives less accurate measures of the signal decay rate.

For larger modulation amplitudes in this data set, there was no sudden drop in decay rate indicating modulation above the island tune - each set of modulations destroyed the persistent signal at progressively smaller and smaller frequencies. A pair of these cutoff frequencies for higher modulation amplitudes are also shown in the tune modulation plane of Figure (5.24), just below the small-angle stability curve predicted by theory.

Another consistency with the results of Chapter 4 and simulation is the sudden 


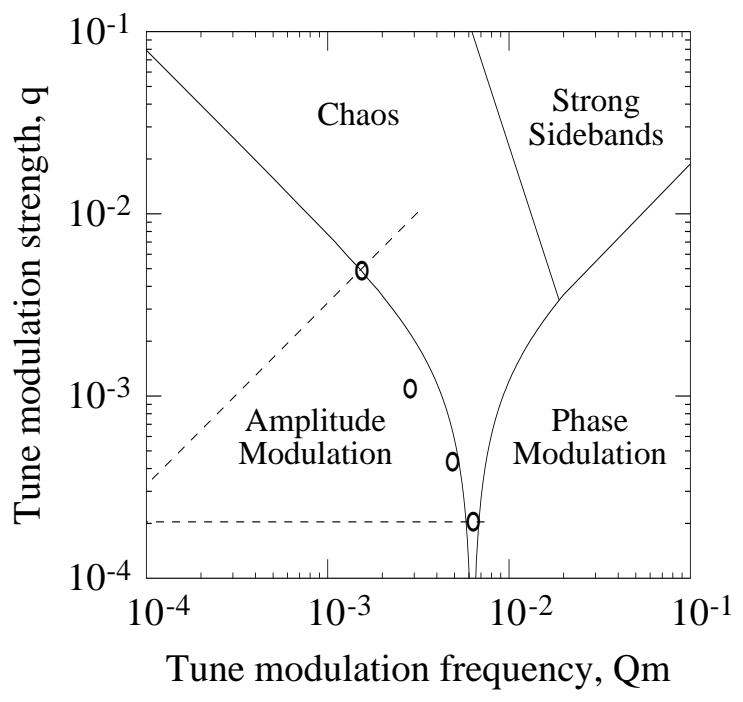

Figure 5.24: The tune modulation parameter plane, with stability lines as predicted by theory and experimental chirps for the 91_0 sextupole configuration. $Q_{\mathrm{I}}$ has been set to the value $6.3 \cdot 10^{-3}$ here. The diagonal chirp corresponds to the data in Figure 5.11; the horizontal chirp corresponds to the $0 \mathrm{~Hz}$ to $350 \mathrm{~Hz}$ chirp shown in Figure 5.23.

stability at modulation frequencies above the island tune, as depicted in the OdChirp simulation results of Figure (4.4). The slowly rising response at frequencies lower than the island tune is also expected because the ensemble of particles filling the resonance island has a frequency distribution which is the same as that of an ensemble of stable pendula, varying from zero frequency at the separatrix to the island tune. Naively, little response is expected above the island tune, because there are no particles oscillating with this frequency to respond to the external drive. Another way to examine the island tune is to start the chirp above the island frequency and chirp downwards - a sudden sharp rise in response at the island tune should be observed. However, technical problems in the tune modulation controls prevented use of this sort of modulation during this experimental run. 


\subsubsection{Chirp Simulation and Comparison to Data}

Simulations were performed of this modulated island system using the program OdChirp, which uses the tune modulation lattice of OdTrack and Odfp to simulate E778 conditions. This program launches an array of initial conditions, typically $10^{3}$, populating a transverse resonance island in much the same way resonance islands were populated to produce persistent signals in the experiment. After allowing untrapped particles to decohere over approximately 500 turns, tune modulation was applied in the exact same fashion as in the experimental run, with the same modulation ramps, strengths and frequencies. The turn-by-turn position of the centroid of the distribution of phase-locked particles was recorded.

This procedure allowed application of exactly the same data reductions as those applied to the actual data, and comparison of the simulated and actual frequency domain response. Because the island amplitude and base tune were well-defined from knowledge of the machine base tune and kicker settings, they could be inserted as known amplitudes, base tunes and octupole strengths for the simulation. The only free parameter then left in the simulation is the decapole strength; variation of this strength allowed a one-parameter fit to the data to produce a final value of the island tune, $Q_{\mathrm{I}}$. An excellent fit was produced for an island tune $Q_{\mathrm{I}}=6.3 \cdot 10^{-3}$ or $296 \mathrm{~Hz}$, in moderately good agreement with the predicted value of $335 \mathrm{~Hz}$ from particle tracking of the 91_0 lattice. Using this value of the island tune, a tune modulation plane can be drawn similar to Figure (4.1) showing excellent agreement for higher amplitude tune modulation chirps. 


\section{CHAPTER 6 PERSISTENT SIGNALS IN TWO TRANSVERSE DIMENSIONS}

In this chapter, we extend the previous one-dimensional analysis of unmodulated resonance islands presented in Chapter 3 to two transverse dimensions. The obvious disadvantage of the previous methods is that they offer little or no hope of investigating two-dimensional resonance strengths experimentally, as persistent signals are not present when a beam is kicked onto a single coupling or difference resonance. However, if the crossing point of two linearly independent resonances is examined with detuning present in both planes, this chapter shows that one expects to find locally phase-locked motion (and corresponding persistent signals) in both planes created by the interaction of the pair of resonances.

In $\S 6.1$ a dual-resonance N-turn map is derived from the results of previous chapters, and predictions for both the stable fixed points of such a map and the island tunes of linearized motion around these fixed points are presented. These results are compared to particle tracking in the octupole-decapole lattice in $\S 6.2$, where the inadequacy of first-order perturbation theory in predicting small oscillation frequencies for this system is demonstrated. $\S 6.3$ discusses this and other possible methods of measuring two-dimensional resonance strengths, and outlines a possible experiment to observe two-dimensional persistent signals, including realistic requirements on availability of controlled nonlinearities.

\subsection{THEORETICAL PREDICTIONS}

Here we examine the crossing point of a one-dimensional resonance and a twodimensional resonance. For the sake of simplicity and relevance to the previous chapters, the one-dimensional resonance is assumed to be $5 Q_{x}$, while the twodimensional resonance is completely general, but specified by $k Q_{x}+l Q_{y}$. Sac- 
rificing other resonance terms with the assumption that they are either small or average out over future summations via the Dirichlet kernel suppression, we can write a one-turn two-dimensional dual resonance Hamiltonian:

$$
\begin{aligned}
H_{1}\left(\psi_{x}, J_{x}, \psi_{y}, J_{y}\right)= & 2 \pi Q_{x 0} J_{x}+2 \pi Q_{y 0} J_{y} \\
& +\frac{1}{2} \alpha_{x x} J_{x}^{2}+\alpha_{x y} J_{x} J_{y}+\frac{1}{2} \alpha_{x y} J_{y}^{2} \\
& +V_{50}\left(J_{x}\right) \cos \left(5 \psi_{x}+\phi_{50}\right) \\
& +V_{k l}\left(J_{x}, J_{y}\right) \cos \left(k \psi_{x}+l \psi_{y}+\phi_{k l}\right) .
\end{aligned}
$$

Here $V_{50}$ and $V_{k l}$ contain the functional dependence of the resonance strengths on the actions $J_{x}$ and $J_{y}$, since these dependences cannot be expected to be as simple as the one-dimensional case. Note that the detuning contains a nonlinear coupling term $\alpha_{x y}$, even though the absence of linear coupling is still assumed.

Horizontal motion is expected to be resonant when the horizontal tune is on the $5 Q_{x}$ resonance; for the vertical motion to also fall on the two-dimensional resonance, the net phase advance within the angle dependence of this resonance term must be near some multiple of $2 \pi$. This gives the natural base tunes for this investigation:

$$
Q_{x 0}=\frac{M_{x}}{5}+\delta_{x}, \quad Q_{y 0}=\frac{M_{y}}{l}-\frac{k M_{x}}{5 l}+\delta_{y},
$$

where $M_{x}$ and $M_{y}$ are integers. Here the $\delta_{i}$ are again assumed to be small, and motion will approximately repeat itself in the 4-dimensional phase space every $N \equiv 5 k l$ turns. (This is the maximum value necessary for repetition; the motion will repeat faster than this if 5 and $k l$ are commensurate.) The actions are invariant to zeroth order, and we can sum over $N$ turns to find the first-order $N$-turn Hamiltonian,

$$
\begin{aligned}
H_{N}\left(\psi_{x}, J_{x}, \psi_{y}, J_{y}\right)= & 2 \pi \delta_{x} J_{x}+2 \pi N \delta_{y} J_{y} \\
& +\frac{N}{2} \alpha_{x x} J_{x}^{2}+N \alpha_{x y} J_{x} J_{y}+\frac{N}{2} \alpha_{y y} J_{y}^{2} \\
& +N V_{50}\left(J_{x}\right) \cos \left(5 \psi_{x}+\phi_{50}^{\prime}\right) \\
& +N V_{k l}\left(J_{x}, J_{y}\right) \cos \left(k \psi_{x}+l \psi_{y}+\phi_{k l}^{\prime}\right) .
\end{aligned}
$$


Here the phases of the nonlinearities have absorbed the shift from the summation: $\phi_{50}^{\prime} \equiv \phi_{50}+5 \pi(N-1) \delta_{x}$ and $\phi_{k l}^{\prime} \equiv \phi_{k l}+\pi k(N-1) \delta_{x}+\pi l(N-1) \delta_{y}$.

Equation (6.3) has fixed points akin to the one-dimensional fixed points of the analysis in Chapter 2. These can be found by examining the difference equations of motion generated by this Hamiltonian, and setting them to zero at the fixed points of the map.

$$
\begin{aligned}
\Delta \psi_{x}=0 & \rightarrow 2 \pi \delta_{x}+\alpha_{x x} J_{x, f p}+\alpha_{x y} J_{y, f p}+\vartheta(V)=0, \\
\Delta \psi_{y}=0 & \rightarrow 2 \pi \delta_{y}+\alpha_{x y} J_{x, f p}+\alpha_{y y} J_{y, f p}+\vartheta(V)=0, \\
\Delta J_{x}=0 & \rightarrow 5 \psi_{x, f p}+\phi_{50}^{\prime}=2 \pi k_{x}, \\
\Delta J_{y}=0 & \rightarrow k \psi_{x, f p}+l \psi_{y, f p}+\phi_{k l}^{\prime}=2 \pi k_{y} .
\end{aligned}
$$

Assuming the detuning terms are stronger than the resonance driving terms, as before, Equations (6.4a) and (6.4b) are coupled linear equations for the fixed point actions, and so they can be easily solved to find

$$
\left(\begin{array}{l}
J_{x, f p} \\
J_{y, f p}
\end{array}\right)=\frac{2 \pi}{\alpha_{x y}^{2}-\alpha_{x x} \alpha_{y y}}\left(\begin{array}{cc}
\alpha_{y y} & -\alpha_{x y} \\
-\alpha_{x y} & \alpha_{x x}
\end{array}\right)\left(\begin{array}{l}
\delta_{x} \\
\delta_{y}
\end{array}\right)
$$

if they are not degenerate; if they are indeed degenerate, no action fixed points exist. Equations (6.4c) and (6.4d) give the fixed point phases:

$$
\begin{aligned}
\psi_{x, f p} & =\frac{\pi k_{x}-\phi_{50}^{\prime}}{5} \\
\psi_{y, f p} & =\frac{\pi k_{y}-\phi_{k l}^{\prime}-k \psi_{x, f p}}{l} .
\end{aligned}
$$

Motion around these fixed points can be classified in one of four ways depending on the parity of the integers $k_{x}$ and $k_{y}$ - it is either hyperbolically unstable in both oscillation directions, hyperbolic in one direction and elliptically stable in the other, or elliptically stable in both oscillation directions. This last case is the one that interests us here, because phase localization in both planes will result in observable persistent signals in both planes. We choose $k_{x}$ and $k_{y}$ even for now, 
with the knowledge that other fixed points can be investigated by changing their parity and thus the signs of the resonance strengths $V_{50}$ and/or $V_{k l}$.

Now transform the coordinates to those centered on one of these fixed points, $\left(\psi_{x}, J_{x}, \psi_{y}, J_{y}\right) \rightarrow\left(\theta_{x}, I_{x}, \theta_{y}, I_{y}\right)$, via the uncoupled two-dimensional generating function

$$
F\left(\psi_{x}, I_{x}, \psi_{y}, I_{y}\right)=\left(I_{x}+J_{x, f p}\right)\left(\psi_{x}-\psi_{x, f p}\right)+\left(I_{y}+J_{y, f p}\right)\left(\psi_{y}-\psi_{y, f p}\right) .
$$

Applying this transformation to the $N$-turn Hamiltonian (6.3) and keeping only first order terms in the nonlinear strengths $V_{k l}$ and $\alpha_{i j}$ gives a $N$-turn Hamiltonian that has the form of a pair of coupled pendula:

$$
\begin{aligned}
H_{N}= & \frac{N}{2} \alpha_{x x} I_{x}^{2}+N \alpha_{x y} I_{x} I_{y}+\frac{N}{2} \alpha_{y y} I_{y}^{2} \\
& +N V_{50}\left(J_{x, f p}\right) \cos \left(5 \theta_{x}\right) \\
& +N V_{k l}\left(J_{x, f p}, J_{y, f p}\right) \cos \left(k \theta_{x}+l \theta_{y}\right) .
\end{aligned}
$$

The resonance strengths $V_{50, f p}$ and $V_{k l, f p}$ are evaluated explicitly at the action fixed points $\left(J_{x, f p}, J_{y, f p}\right)$.

Linearizing the coupled motion given by this Hamiltonian is a tedious but straightforward process. The final result of this linearization gives the motion of the angle variables $\left(\theta_{x}, \theta_{y}\right)$ as

$$
\left(\begin{array}{l}
\ddot{\theta}_{x} \\
\ddot{\theta}_{y}
\end{array}\right)=N^{2}\left(\begin{array}{ll}
M_{11} & M_{12} \\
M_{21} & M_{22}
\end{array}\right)\left(\begin{array}{c}
\theta_{x} \\
\theta_{y}
\end{array}\right),
$$

where time derivatives are taken with respect to $N$-turn motion, and the individual matrix elements of the coupled motion are

$$
\begin{aligned}
& M_{11}=25 \alpha_{x x} V_{50, f p}+k V_{k l, f p}\left(k \alpha_{x x}+l \alpha_{x y}\right) \\
& M_{12}=l V_{k l, f p}\left(k \alpha_{x x}+l \alpha_{x y}\right) \\
& M_{21}=25 \alpha_{x y} V_{50, f p}+k V_{k l, f p}\left(k \alpha_{x y}+l \alpha_{y y}\right) \\
& M_{22}=l V_{k l, f p}\left(k \alpha_{x y}+l \alpha_{y y}\right) .
\end{aligned}
$$


There are two normal mode "island" frequencies of the two coupled oscillators in Equation (6.9). These could be found by diagonalizing the matrix $M$, as could the eigenvectors that represent the normal modes of oscillation. However, since we are primarily concerned with the island frequencies here, we can find those by calculating the eigenvalues of $M$ directly. Note that if either or both of these eigenvalues are negative or imaginary, local motion here is unstable and expansion should proceed around a different fixed point, changing either or both of the signs of the resonance strengths in the N-turn Hamiltonian (6.8).

For completeness, the island tunes corresponding to this motion are

$$
\begin{aligned}
{\left[2 \pi Q_{\mathrm{I}(1,2)}\right]^{2}=} & \frac{A_{50} V_{50, f p}+A_{k l} V_{k l, f p}}{2} \\
& \pm \frac{\sqrt{\left(A_{50} V_{50, f p}+A_{k l} V_{k l, f p}\right)^{2}-\Delta_{l} V_{50, f p} V_{k l, f p}}}{2}
\end{aligned}
$$

where the amplitudes $A_{50}, A_{k l}$ and $\Delta_{l}$ are defined by

$$
\begin{aligned}
& A_{50}=25 \alpha_{x x}, \\
& A_{k l}=\left(k^{2} \alpha_{x x}+2 k l \alpha_{x y}+l^{2} \alpha_{y y}\right), \\
& \Delta_{l}=100 l^{2}\left(\alpha_{x x} \alpha_{y y}-\alpha_{x y}^{2}\right) .
\end{aligned}
$$

Assuming that the resonance strengths $V_{50}$ and $V_{k l}$ are both positive, these island tunes are both positive real if $\Delta_{l}$ lies within the range

$$
0 \leq \Delta_{l}(\text { stable }) \leq \frac{\left(A_{50} V_{50, f p}+A_{k l} V_{k l, f p}\right)^{2}}{V_{50, f p} V_{k l, f p}}
$$

An amazing prediction of Equation (6.11) is that if all detuning strengths scale similarly according to some nonlinearity strength, and the resonance strengths scale similarly according to another nonlinearity strength, then the local stability of this system is independent of both these resonance strengths to first order.

One easily testable scaling of the island tune prediction is that the island tunes should scale as the square root of the nonlinearity strength if the resonances are 
driven to first order in that strength. The sum of the squares of the island tunes is also a more easily accessible quantity to test against theory; from Equation (6.10a) this is found to be

$$
\left[2 \pi Q_{\mathrm{I}, 1}\right]^{2}+\left[2 \pi Q_{\mathrm{I}, 2}\right]^{2}=A_{50} V_{50, f p}+A_{k l} V_{k l, f p},
$$

which scales as the product of the nonlinear strengths driving the detuning and the resonances.

\subsection{TRACKING OF TWO-DIMENSIONAL RESONANCE ISLANDS AND PERSISTENT SIGNALS}

The question remains as to whether such two-dimensional resonance islands can be observed in a tracking code, and whether the first-order analysis suffices to predict their island tunes and locations in phase space. Using the octupoledecapole model and a program Od2Track, which was written to track this model in both transverse dimensions, two-dimensional detuning parameters $\alpha_{i j}$ are first measured as functions of amplitude in both planes and compared to first-order predictions for the octupoles. A working point must be chosen where the $5 Q_{x}$ resonance and another resonance driven to first order by the decapole cross; this point in the tune plane $\left(Q_{x 0}, Q_{y 0}\right)$ should also be free of second-order octupole resonances, since such resonances are not calculated in a first-order analysis. Next, two-dimensional resonance islands are found and their fixed point amplitudes and phases are compared to theoretically predicted values. Island tunes are found for the normal mode oscillations within these resonance islands and compared against

both absolutely predicted values and scaling with the decapole strength $\tilde{b}_{4}$.

From the discussion of the octupole-decapole lattice in Chapter 3 we can write the first-order dependences of the Hamiltonian parameters $\alpha_{i j}$ and $V_{k l}$ on the 


\begin{tabular}{|l|c|c|}
\hline Tracking Parameter & Symbol & Value \\
\hline \hline Octupole strengths (each) & $\tilde{b}_{3}$ & 0.0100 \\
Decapole strength & $\tilde{b}_{4}$ & 0.0015 \\
Horizontal Tune Offset & $\delta_{x}$ & 0.0050 \\
Vertical Tune Offset & $\delta_{y}$ & 0.0050 \\
Horizontal Base Tune & $Q_{x 0}$ & 20.6050 \\
Vertical Base Tune & $Q_{y 0}$ & 20.6550 \\
\hline
\end{tabular}

Table 6.1: Parameters used for two-dimensional persistent signal investigation in the tracking program Od2Track.

lattice parameters listed in Table 6.1. The detuning is driven by the octupoles,

$$
\alpha_{x x}=\alpha_{y y}=-\frac{1}{2} \alpha_{x y}=\frac{9}{4} \tilde{b}_{3}
$$

and the resonances are driven by the decapole:

$$
\begin{aligned}
V_{50} & =\frac{\sqrt{2}}{20} \tilde{b}_{4} J_{x}^{5 / 2} \\
V_{1 \pm 2} & =\frac{\sqrt{2}}{2} \tilde{b}_{4} J_{x}^{1 / 2} J_{y}\left(2 J_{y}-3 J_{x}\right), \\
V_{3 \pm 2} & =-\frac{\sqrt{2}}{2} \tilde{b}_{4} J_{x}^{3 / 2} J_{y}, \\
V_{1 \pm 4} & =\frac{\sqrt{2}}{4} \tilde{b}_{4} J_{x}^{1 / 2} J_{y}^{2} .
\end{aligned}
$$

These detuning parameters give the action dependences of the tunes:

$$
\left(\begin{array}{l}
Q_{x} \\
Q_{y}
\end{array}\right)=\left(\begin{array}{l}
Q_{x 0} \\
Q_{y 0}
\end{array}\right)+\frac{9 \tilde{b}_{3}}{8 \pi}\left(\begin{array}{cc}
1 & -2 \\
-2 & 1
\end{array}\right)\left(\begin{array}{l}
J_{x} \\
J_{y}
\end{array}\right)
$$

The resulting "footprint" of this tune shift on the tune plane is a rhombus with an oblique opening angle of $143^{\circ}$; this is shown in Figure (6.1) as contours of constant amplitude. Circles in this figure are tunes for tracked particles, with actions in each plane ranging from 0.0 to 4.0 in steps of 0.4 ; excellent agreement is shown for the situation where particles are not affected by nearby strong resonances. Note that the decapole was not turned off for this check, since the second-order tuneshift from the decapole is expected to be small compared to that of the octupoles. 


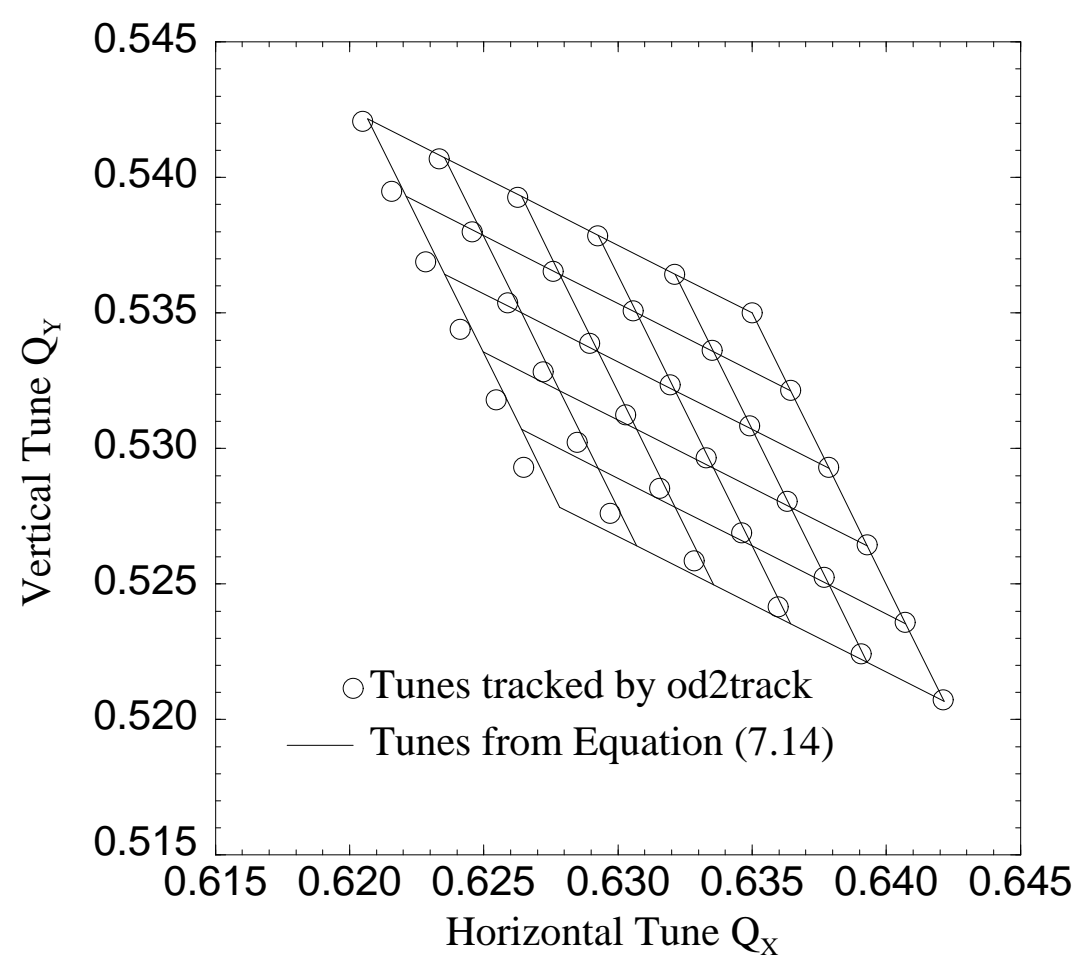

Figure 6.1: Comparison of octupole detuning as predicted by Equation (6.14) to tracking of the octupole-decapole lattice. Lines are predicted contours of constant action, ranging from 0 to 2 in steps of 0.2 . Circles represent tracked tune offsets. Magnet strengths are as in Table (6.1); base tunes are $Q_{x 0}=20.66, Q_{y 0}=20.64$, far from prevailing resonances.

Now the tune plane must be investigated for an optimal working point for this tracking experiment to find 2-dimensional persistent signals. For agreement with the previous theory, the resonances under investigation should both be driven to first order in the decapole strength, and one should be the horizontal resonance $5 Q_{x}$. Since the octupoles are much stronger than the decapoles in this simulation, creating strong stabilizing detuning, all resonances up to second order in octupole strengths should be avoided. Examination of the tune plane of Figure (6.2), with first order decapole resonances represented by solid lines and second order octupole resonances represented by dashed lines, shows a promising working point at the 


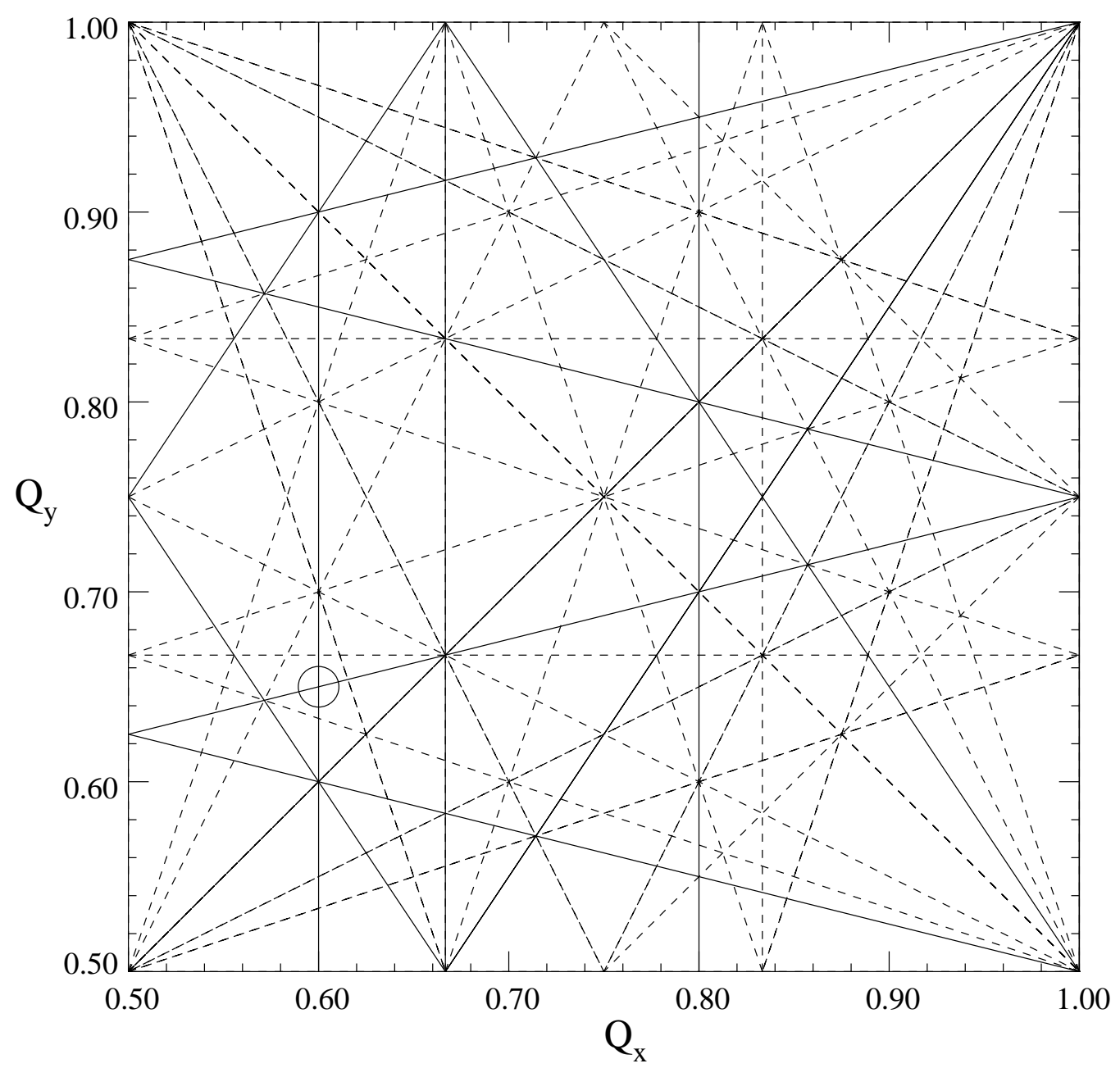

Figure 6.2: The tune plane for the octupole-decapole lattice. Resonances driven to first order in the decapole strength are represented by solid lines; resonances driven to second order in the octupole strength are represented by dashed lines. The circle marks the chosen resonant point, $\left(Q_{x, \text { res }}, Q_{y, \text { res }}\right)=(20.60,20.65)$, for a 2 -dimensional decapole resonance crossing simulation. 
resonant tunes

$$
Q_{x, \text { res }}=0.600=\frac{3}{5}, \quad Q_{y, \text { res }}=0.650=\frac{13}{20}
$$

At this point the $5 Q_{x}$ and $Q_{x}-4 Q_{y}$ resonances cross, so $k=1$ and $l=-4$. Since both resonance tune denominators are commensurate it suffices to take a Poincaré surface of section every 20 turns, instead of every 100 turns as would be suggested by the previous discussion.

For the tracking conditions of Table (6.1) and previously calculated detuning coefficients, the fixed-point actions are predicted by Equation (6.5) to be $J_{x, f p}($ theory $)=J_{y, f p}($ theory $)=1.396$. Instead of going through tedious calculations necessary to predict the fixed point phases, these actions were used as initial conditions with which to track at a variety of initial phases, searching for initial conditions that at least lay within the separatrix of the four-dimensional phase space resonance islands. Such a set of phases, $\left(\psi_{x}=0.2, \psi_{y}=0.3\right)$, was easily found after a small range of initial phases were checked, since the long-term tunes of resonantly trapped particles will be exactly the resonant tunes.

A turn-by turn Poincaré plot of the four-dimensional phase space motion of this set of initial conditions is pictured at the top of Figure (6.3). This plot certainly shows that there is some sort of phase space structure present, but it is difficult to determine whether or not there is resonant motion. Various types of other tools can be used to visualize different sections of this phase space, including three-dimensional projections instead of the two-dimensional projections that are normally used (Holt et. al. 1992). For this analysis, however, a simpler technique is available, which consists of taking a Poincare surface of section every $N=20$ turns of the motion. If the motion is truly resonant, then this stroboscopic view will show phase-locked motion, and a persistent signal would be visible for a real distribution of particles launched on and near this orbit. This is indeed the case 

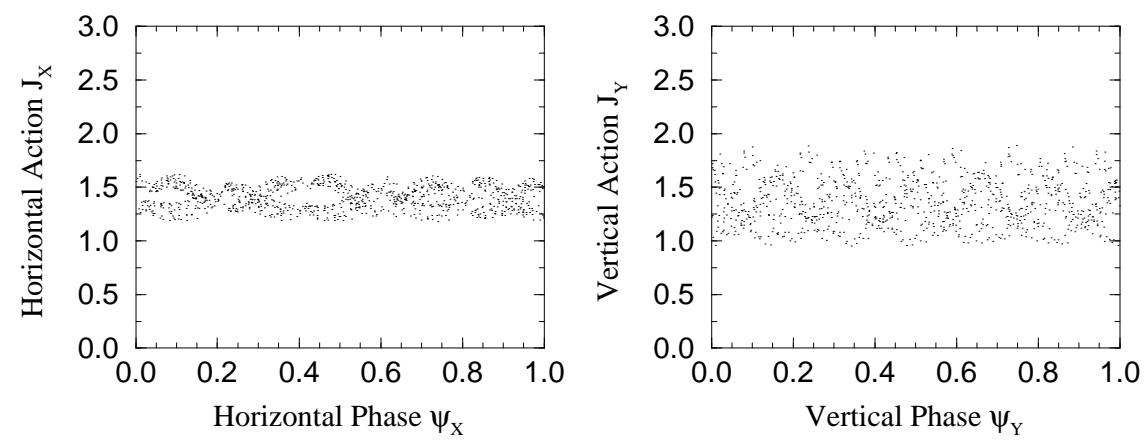

Only plot every 20th tracked turn

$\Downarrow$
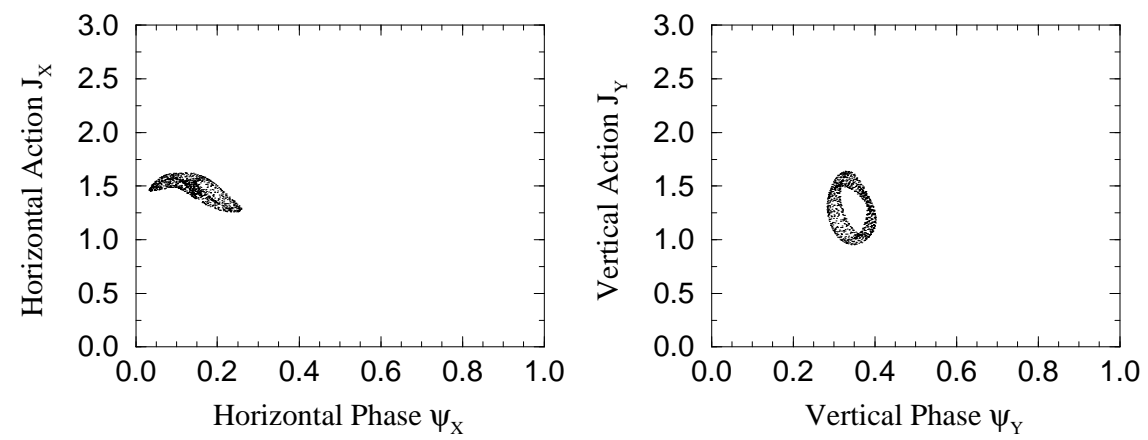

Find centroid of separatrix and retrack

$\Downarrow$
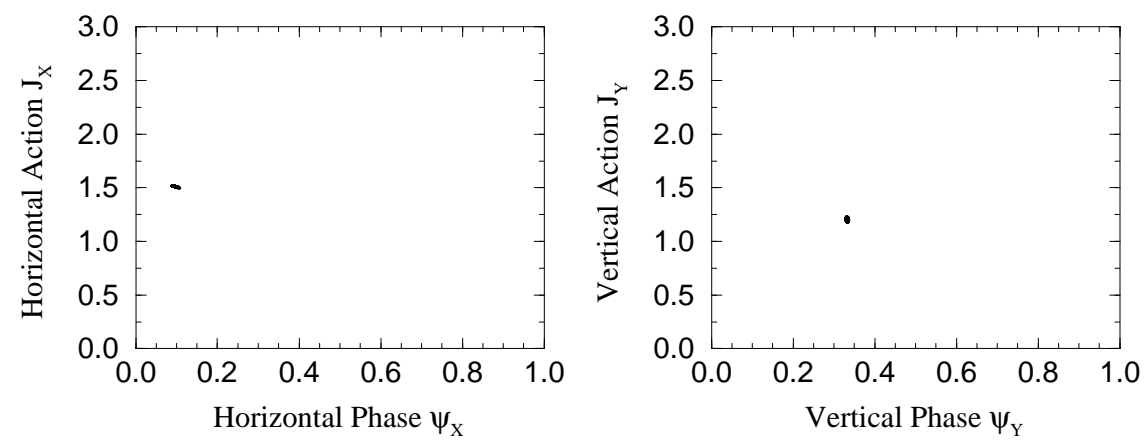

Figure 6.3: Four-dimensional phase space projections used in finding twodimensional resonance islands and persistent signals. Coherent motion is evident in all three plots. 

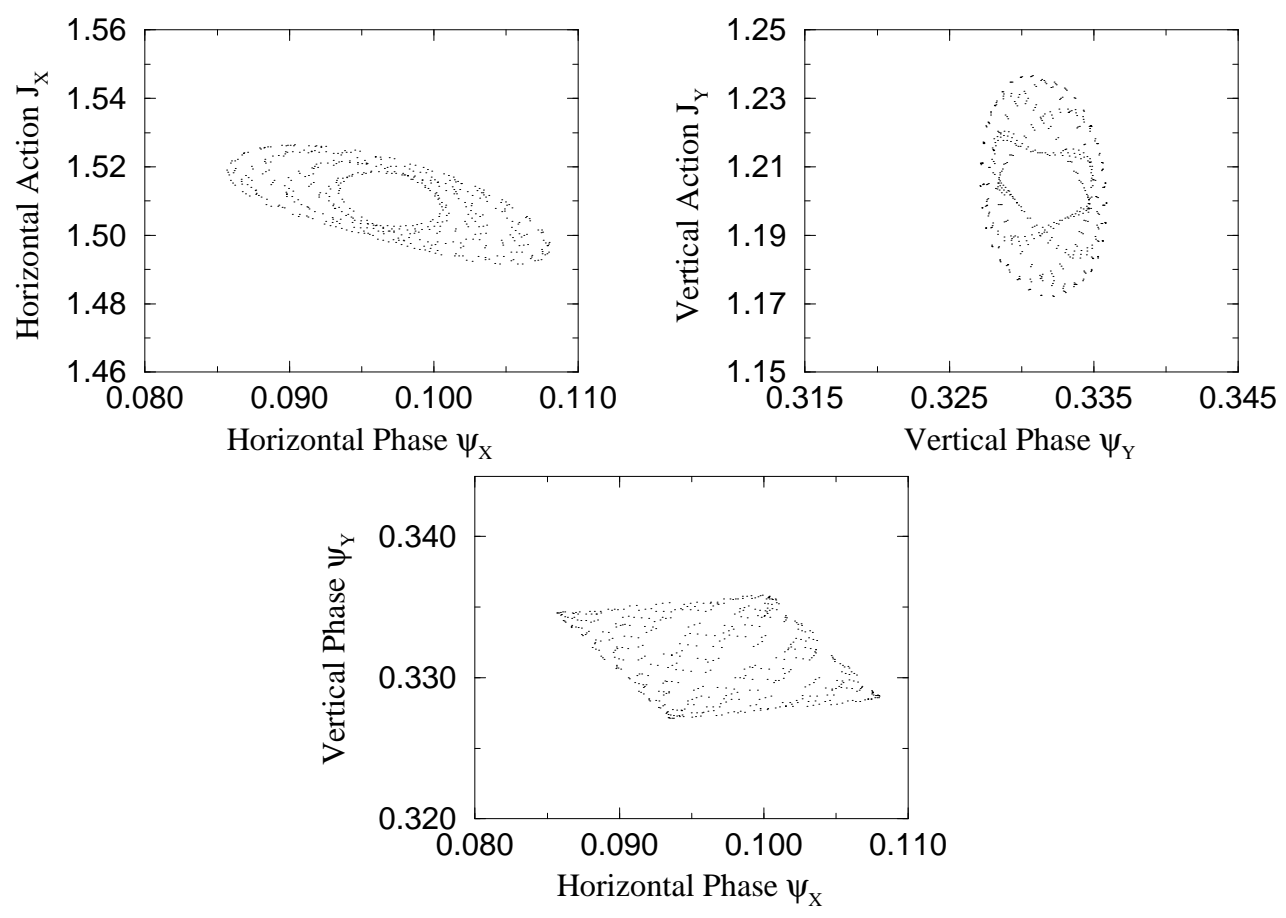

Figure 6.4: Close-up of tracking very near the fixed point of the twodimensional resonance islands, plotted every 20th turn.

for the above initial conditions, as can be seen in the middle of Figure (6.3) - a resonant orbit appears.

Once any sort of resonant orbit has been found, an iterative process can be used to find the actual fixed point of the lattice used for tracking. The centroid of the distribution in each plane is calculated for a number of turns much larger than the expected island periods, thus averaging over many rotations in the island, and these centroids are substituted back into the tracking program as new initial coordinates. This is much less efficient than the more sophisticated methods used in the one-dimensional fixed-point location algorithm of Chapter 4, but is much more easily implemented than would be the corresponding 2-dimensional extension of such an approach. Four iterations yield the fixed point of the lattice 
described in Table (6.1) to four significant figures,

$$
\begin{aligned}
& \left(\psi_{x, f p}, J_{x, f p}\right)=(0.0967,1.5104), \\
& \left(\psi_{y, f p}, J_{y, f p}\right)=(0.3314,1.2040),
\end{aligned}
$$

which is gives modest (10-15\%) agreement with the fixed point amplitudes predicted by theory. The resulting phase space of an intermediate step of this iterative process is shown at the bottom of Figure (6.3), and magnified in Figure (6.4) to show the character of motion for small amplitudes $I_{i}$ near the fixed point. This motion falls on a two-dimensional torus embedded in the four-dimensional phase space, since there are two invariants corresponding to the locally phase-locked motion in each transverse dimension.

Once the fixed point has been found, the island tunes can be measured from tracking data by taking 20-turn stroboscopic data from either plane and performing an FFT on some portion of data with $2^{n}$ data points. Taking 16384 data points suffices for a measurement of these tunes to an accuracy of approximately $10^{-4}$, and gives $Q_{I, 1}=2.1 \cdot 10^{-4}$ and $Q_{I, 2}=8.7 \cdot 10^{-4}$ for the data presented in Figure (6.4).

For a large range of decapole strengths, up to strengths comparable to the octupole strength, the island tunes are displayed in Figure (6.5). One peak from the FFT of small oscillatory motion scales according to $\tilde{b}_{4}^{1 / 2}$ as predicted, but the other appears to scale linearly. No other significant peaks appear in an examination of the FFT of this motion, indicating that the linear scaling is not an anomolous peak created by interference of the natural oscillation frequencies. There is also no evidence that these peaks are aliased from higher frequencies, as they each approach zero at zero decapole strengths.

The island tunes predicted by Equations (6.10a) and (6.10b) are also shown in Figure (6.5), and are nearly three to five times larger than the island tunes found by tracking. The inconsistency between theory and tracking for the resonant 


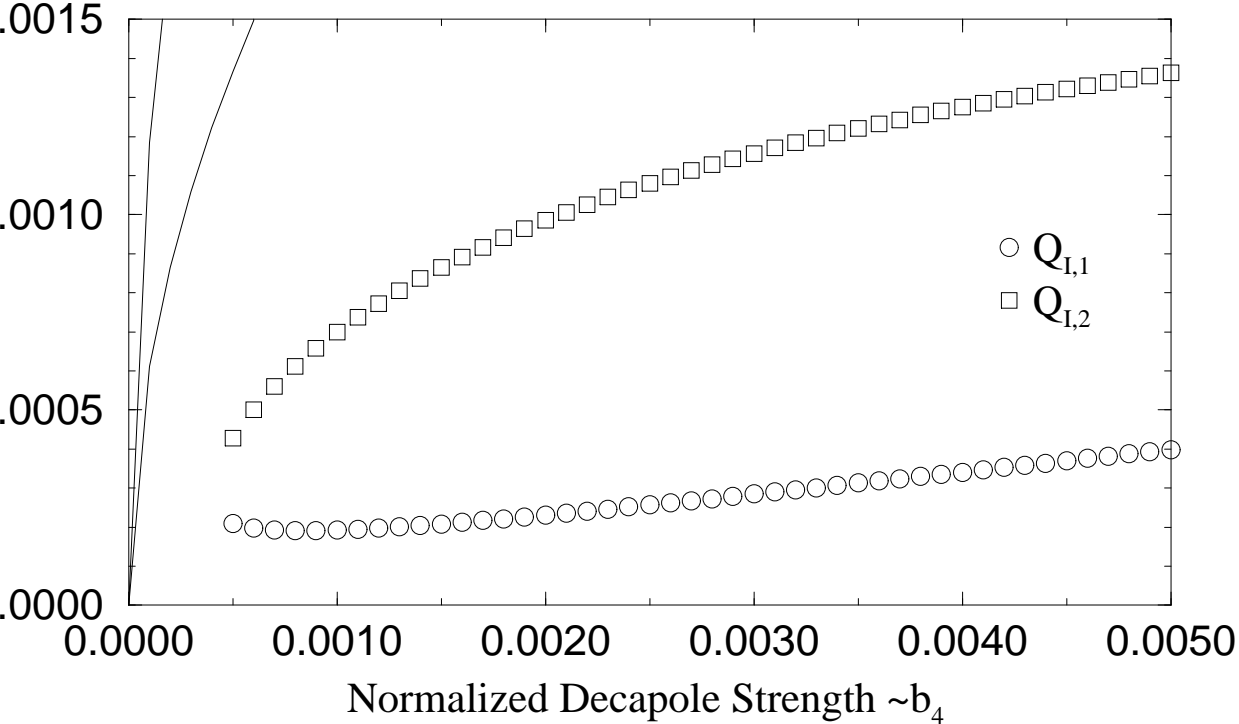

Figure 6.5: Two-dimensional island tunes $\left(Q_{I, 1}, Q_{I, 2}\right)$ measured as a function of decapole strength $\tilde{b}_{4}$ in the tracking program Od2fp. All other lattice parameters for this tracking are as listed in Table (6.1). Lines are theoretical predictions for island tunes.

crossing point small oscillation tunes also appears at other values of the octupole strength as well as other resonance crossing points where second-order octupole resonances are present. This inability of first-order perturbation theory to predict the small-oscillation motion in these areas of the tune plane is a strong indication that such approaches fail to give meaningful results at the crossing of several resonances. Higher order resonances are undoubtedly present at these crossing points, and possibly higher orders of perturbative magnet strengths must be introduced to reconcile theory with tracking. However, with the addition of other resonances the linearization process previously described becomes difficult, particularly because the equations defining the fixed-point phases are transcendental.

\subsection{A POSSIBLE EXPERIMENT}

How might these two-dimensional persistent signals be observed in a real accel- 
erator? First, one must operate with a small beam — much smaller, in fact, than any available at the Fermilab Tevatron, since the Tevatron beam size is typically one-tenth of the physical aperture. Use of a small cooled beam would be optimal, since this would maximize the proportion of the beam actually trapped within the four-dimensional phase space well and produce the highest signal-to-noise ratio for persistent signals in both planes.

Kicks to move the beam onto the two-dimensional resonance islands cannot be applied independently in each plane with linear coupling present; such linear coupling would rapidly transfer energy in the kicked plane to energy in the unkicked plane, increasing the beam emittance in the unkicked dimension. Linear coupling can be removed globally by adjustment of skew quadrupoles, a standard procedure that was also performed in E778 for the explicitly horizontal one-dimensional kick.

The lattice must be studied extensively for nonlinearities before the study is begun, and both linear and nonlinear coupling must be removed as much as possible. This implies measuring the detuning coefficients $\alpha_{x x}, \alpha_{y y}$, and $\alpha_{x y}$. The nonlinear coupling $\alpha_{x y}$ can be measured by kicking the beam in one transverse plane and observing the tune shift in the unkicked plane, far from strong resonances. Measurement of three or four points should suffice for a reasonable measurement, and then octupoles (if present) can be adjusted to remove this component. When the nonlinear coupling has been removed, turn-by-turn data can be taken in both planes with separate kicks to test the turn-by-turn data acquisition system and measure the detuning coefficients $\alpha_{x x}$ and $\alpha_{y y}$, as has been done previously in E778.

Once the detuning coefficients are known, the actual amplitudes of the resonance islands can be calculated to first order, and base tunes can be chosen to optimize the positions of the four-dimensional phase space fixed points. Kicking first in the 
plane where the one-dimensional resonance is present, a systematic scan of various kick amplitudes can be performed, and persistent signals should be evident when the beam is kicked onto the one-dimensional resonance island. Up to this point such an experiment reproduces previous persistent signal results of E778 without tune modulation, as well as those of Experiment CE22 at IUCF.

Once the kick amplitude has been found which consistently populates the onedimensional resonance island, another kick in the opposite plane can be inserted in the cycle after the first kick. If linear and nonlinear coupling have been sufficiently minimized this kick does not couple into motion in the opposite the plane which has already been resonantly captured, and so it does not disturb this motion. A similar scan of various kick amplitudes can then be performed in this plane, searching for production of another persistent signal.

For the purposes of practical measurement of the strengths of two-dimensional resonances this method is most probably inadequate, since the crossing points where two-dimensional persistent signals are present are also crossed by many other resonances which affect the motion around the fixed point. This is evident in the failure of first-order perturbation theory to predict island tunes. Other techniques involving observation of orbit distortions in four-dimensional phase space have been investigated, and prove to be more promising for this application (Li 1990, Liu 1989). However, such an experiment could be considered a prelude to investigations of modulational diffusion, which provides a mechanism for luminosity and luminosity lifetime limitations in storage rings as described in the next chapter. Modulational diffusion is expected to be present near the intersection of two strong resonances, in the same region of the tune plane that two-dimensional persistent signals could be observed. 


\section{CHAPTER 7 MODULATIONAL DIFFUSION}

In this chapter a summary of the requirements for modulational (thick-layer) diffusion to exist in a particle synchrotron is presented and applied to a simple tune-modulated collider model of the Fermilab Tevatron where the only nonlinearities present are two beam-beam kicks. This is presented as an example of how nonlinearities combined with tune modulation can cause individual particle amplitude growth, leading to emittance growth and possible lifetime limitations in a storage ring.

Modulational diffusion has been the subject of many investigations in the past ten years, since it provides a particle loss mechanism in many-dimensional dynamical systems such as particle accelerators over timescales that are longer than those from pure resonant loss (typically hundreds of turns), but shorter than the timescales of Arnol'd (or thin-layer) diffusion (typically hundreds of millions of turns). Most of the salient features and quantitative analysis can be found in assorted publications (Chirikov et. al. 1985, Vivaldi 1984, Lichtenberg and Lieberman 1983); in $\S 7.1$ the requirements for modulational diffusion to exist in a synchrotron are outlined, and the qualitative characteristics of amplitude growth created by this diffusion are described. The simulation lattice, a mockup of the Fermilab Tevatron collider lattice, and the beam-beam force are described in $\S$ 7.2. The results of Evol simulation of such a circumstance in an area of phase space where modulational diffusion is expected are described in $\S 7.3$, which shows that amplitude growth in this circumstance is exponential instead of root-time as classically predicted by diffusion theory. These results and possible future directions are summarized in $\S 7.4$. 


\subsection{CHARACTERISTICS OF MODULATIONAL DIFFUSION}

Consider the one-dimensional tune modulation parameter plane of Figure (4.1). For appropriate tune modulation parameters falling within the "Chaos" region, sets of sidebands are created and overlap with the primary resonance and create a band of chaotic motion. Particles located at amplitudes within this chaotic band have phases that oscillate highly irregularly; in modulational diffusion models this chaos serves as a noise source for regular motion in the other transverse plane, coupled to this phase through a weak nonlinear coupling resonance.

Here we assume that there is horizontal tune modulation creating a localized chaotic region in the horizontal phase space and examine what would nominally be regular motion in the vertical dimension, influenced by one or more of these "weak" coupling resonances. (For these purposes such resonances are considered to be "weak" if their amplitudes are much smaller than that of the primary resonance in the horizontal plane driving the horizontal stochasticity.) The motion in the vertical plane is now that of a very weakly driven oscillator, where the driving force is now chaotic due to its weak coupling to the horizontal stochastic motion. Such motion is similar to that of a random walk problem; the stochastically driven vertical motion can "diffuse" out to large amplitudes in finite time.

Chirikov, Lieberman, Vivaldi and Shepelyanski (1985) write the Hamiltonian for the standard modulational diffusion model as

$$
\begin{aligned}
H_{1}\left(\theta_{x}, I_{x}, \theta_{y}, I_{y}\right)= & \frac{1}{2} I_{x}^{2}-\epsilon \cos \left[(k+1) \theta_{x}+\lambda \sin \Omega t\right]+ \\
& \frac{1}{2} I_{y}^{2}-\mu \cos \left[k \theta_{x}+\theta_{y}\right]
\end{aligned}
$$

where $(\theta, I)$ are action-angle variables in each plane. Ignoring the weak twodimensional coupling, the motion in the horizontal dimension of this model is similar to that of the tune-modulated resonance islands in Chapter 4 as given by the N-turn Hamiltonian of Equation (4.27) - $\epsilon$ represents the square of the island 

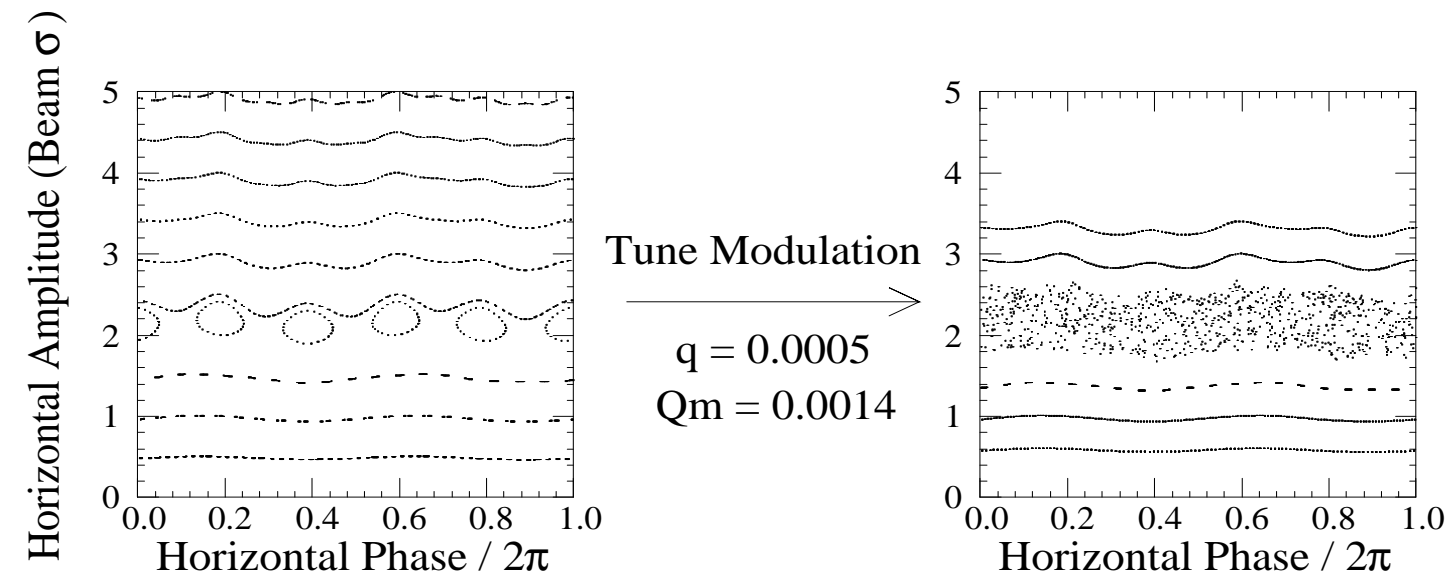

Figure 7.1: 1 dimensional phase space on the $Q_{x}=3 / 5$ resonance, for moderately realistic parameters in the Fermilab Tevatron. Particle tracking is done in Evol with the B0D0 lattice, showing resonance island structures without tune modulation and a thick stochastic band with tune modulation induced by chromaticity.

tune, and $\lambda$ and $\Omega$ represent the strength and frequency of the tune modulation, respectively. Modeling this system with a lattice to be described in the next section produces the one-dimensional phase-space plots shown in Figure (7.1). Motion in the vertical plane in Equation (7.1) is that of a pendulum, weakly coupled to the horizontal motion via the coupling strength $\mu \ll \epsilon$.

A significant difference between the modulational diffusion Hamiltonian (7.1) and the tune modulation nonlinear resonance Hamiltonian (4.27) is the presence of amplitude dependence in the resonance strengths of the latter. In particular, though the horizontal amplitude only varies through the stochastic band, the vertical amplitude growth predicted by modulational diffusion may affect the global motion. Such growth may either carry the horizontal tune off the primary resonance that drives the chaos through detuning, or alter the strength of the coupling resonance, thus changing the vertical amplitude growth rate as the vertical amplitude increases. 


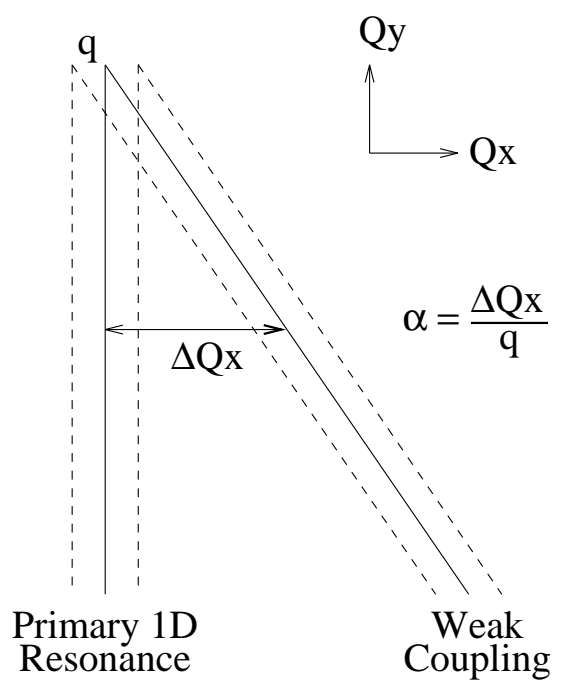

Figure 7.2: Resonance structure for modulational diffusion. $\alpha$ is the horizontal tune distance between the primary driving resonance and the secondary weak coupling resonance, scaled by the modulation depth $q$.

One prediction of modulational diffusion theory is that this diffusive growth in the vertical dimension will scale as the square root of turn number. A diffusion coefficient can be defined,

$$
D \equiv \frac{\left\langle\left(\Delta I_{y}(t)\right)^{2}\right\rangle}{2 T},
$$

where $\Delta I_{y}$ is the vertical action excursion from the initial action and $T$ is the time width of the averaging, in turns. The averaging should be performed over a time $T$ short compared to the vertical diffusion time (so $\Delta I_{y} \ll I_{y}(t=0)$ but long compared to the timescales of motion across the thick horizontal chaotic band (typically hundreds of turns). As the vertical tune is varied along the horizontal one-dimensional resonance, the proximity of the weak coupling resonance changes, as given by the dimensionless quantity

$$
\alpha \equiv \frac{\mid Q_{x} \text { (weak coupling resonance) }-Q_{x} \text { (primary resonance) } \mid}{q} .
$$

A plot of the logarithm of the diffusion coefficient $D$ versus this scaled proximity $\alpha$ shows a series of descending plateaus and sudden drops (Chirikov et. al. 1985). 


\begin{tabular}{|l|c|r|}
\hline Operational Parameter & Symbol & Value \\
\hline \hline Horizontal and vertical chromaticities & $\left(\xi_{x}, \xi_{y}\right)$ & 3.0 \\
Typical momentum offset & $\Delta p / p$ & 0.0003 \\
Synchrotron (modulation) frequency & $Q_{M}$ & 0.00078 \\
Beam-beam linear tune shift per crossing & $\xi$ & 0.005 \\
Revolution frequency & $f_{r e v}$ & $47.7 \mathrm{kHz}$ \\
\hline
\end{tabular}

Table 7.1. Typical Fermilab Tevatron 1992 operational parameters at $900 \mathrm{GeV}$.

This strange structure has sharp drops in $D$ at even integer values of $\alpha$ for the case where both the driving resonance and the coupling resonance are modulated, and it is this sort of structure we attempt to qualitatively reproduce here within the operational framework of the Fermilab Tevatron.

\subsection{THE TEVATRON SITUATION AND AN OPERATIONAL MODEL}

In the Fermilab Tevatron during the 1992 collider run with separators, there were two strong beam-beam interactions every turn - one at the CDF experimental site at ring location $\mathrm{B} 0$ and one at the D0 experimental site. The operating estimate of the linear beam-beam tune shift $\xi$ is approximately $\xi \approx 0.005$ per interaction, and with planned upgrades including the Fermilab Main Injector, this value may very well rise even further (Holmes 1991). With the exceptions of these beam-beam kicks and chromaticity-correction sextupoles (which are neglected for the sake of simplicity of the tracking model), the Tevatron is quite a linear machine, and so its transverse dynamics in this situation here can be modeled extremely simply in the tracking program Evol using only linear phase advances and beam-beam kicks. Typical operating parameters for the 1992 collider run are listed in Table 7.1 .

The base tunes of the Tevatron in typical collider run circumstances are $Q_{x 0} \approx$ 20.586 and $Q_{y 0} \approx 20.575$, running at a horizontal tune between the 12 th order 
resonance, $Q_{x}=20.583$ and the 5th, $Q_{x}=20.600$. For the purposes of this study, however, a worst case scenario is investigated, where the driving resonance for the horizontal stochasticity necessary for modulational diffusion is the 5 th order resonance and single particles are launched at a variety of vertical tunes along this resonance. If $\xi$ ever exceeds .009 with two collisions in the Tevatron, the available space between the 12 th and the 5 th becomes too small for the entire beam, and a significant portion of the beam could be strongly affected by one of these resonances. The relevant portion of the tune plane diagram and the strange shape of the beam-beam footprint are shown in Figure (7.3).

The beam-beam force used here and within the tracking program Evol uses the weak-strong approximation and assumes both beams have round gaussian distributions of equal transverse size $\sigma$. For the horizontal beam-beam kick,

$$
\frac{\Delta x^{\prime}}{\sigma}=\frac{-4 \pi \xi}{\beta_{x}^{\star} R^{2}}\left[1-e^{-R^{2} / 2}\right] \frac{x}{\sigma},
$$

where $x$ is the transverse position relative to the opposing beam center, $x^{\prime} \equiv$ $d x / d s, \beta_{x}^{\star}$ is the beta function at the interaction point, and $R$ is the distance from the center of the opposing beam scaled to the beam size $\sigma$ :

$$
R^{2} \equiv\left(\frac{x}{\sigma}\right)^{2}+\left(\frac{y}{\sigma}\right)^{2}
$$

a kick similar to Equation (7.4) is seen in the vertical plane. Salient features relevant to this study can be noted:

The detuning is drastically different from the model of Equation (7.1), where there is explicitly no coupling other than the weak resonance. The explicit variation of resonance strength with particle amplitude is also a difference between these two models. As vertical amplitude grows in the beam-beam situation, one of two mechanisms will halt modulational diffusion: the vertical amplitude growth will either pull the horizontal tune off the primary driving resonance or it will 


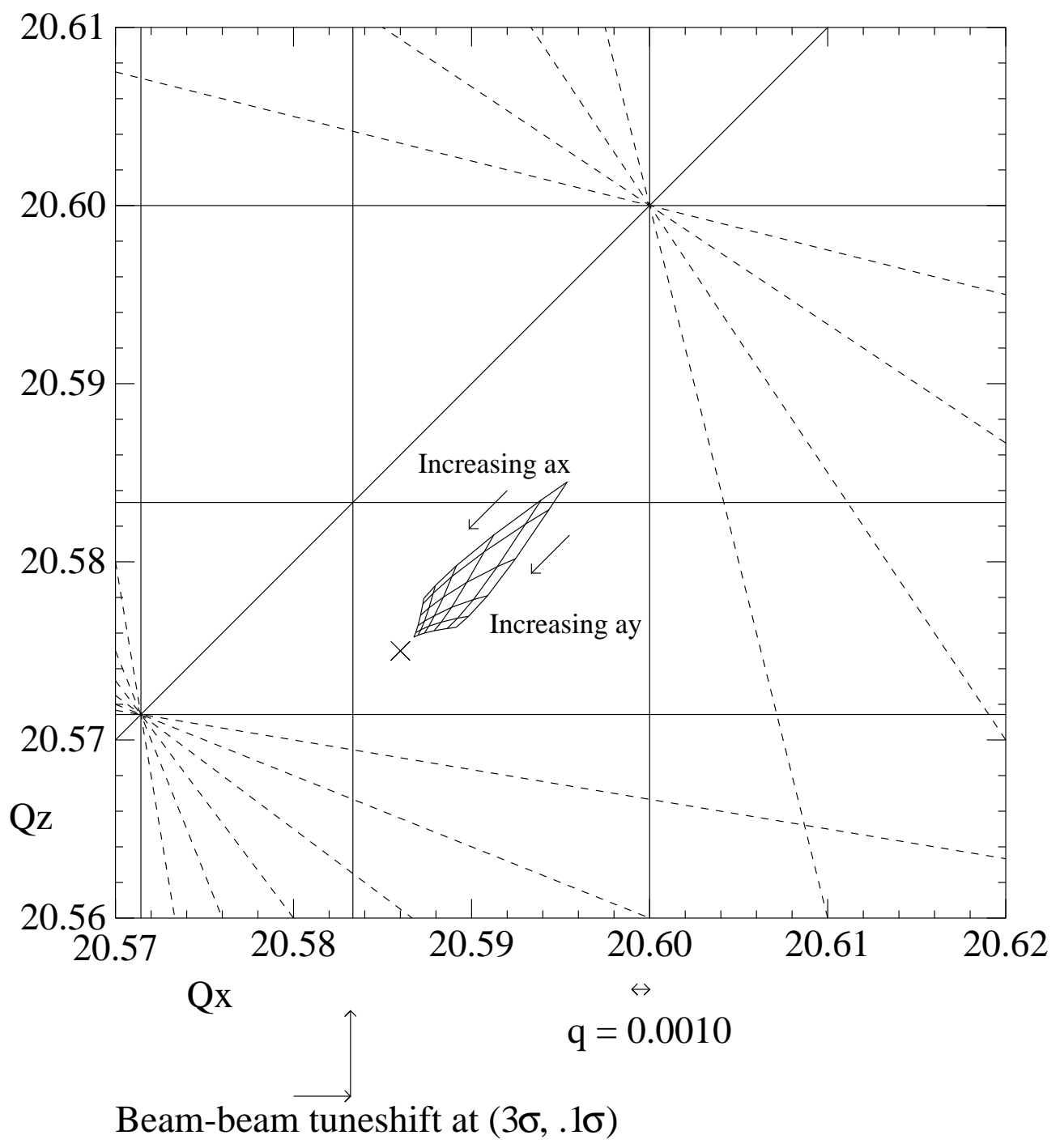

Figure 7.3: The tune plane for typical Fermilab Tevatron 1992 collider operations, showing the 5 th, 7 th and 12 th order resonances. The nominal operating tunes are indicated by the cross, and the beam-beam footprint is shown for $\xi=0.005$ with two collisions at B0 and D0. Footprint contours of constant amplitude range from . $1 \sigma$ to $5.1 \sigma$ in $1 \sigma$ increments. 
- The variation of tune with amplitude (detuning) given by the beambeam force is nonlinear and strongly coupled, quite unlike the octupole detuning observed in the previous chapter.

- From the form of $R$, even-order resonances are driven to first order in $\xi$. Odd-order resonances of order $N$ are driven as even order resonances of order $2 N$.

- Resonance strengths vary with particle amplitude, or action.

- There is no beam-beam tuneshift or resonance driving at infinite amplitudes, so global motion of the unperturbed beam-beam system is stable.

suppress the coupling resonance strength. It remains to be conclusively shown whether such vertical amplitude growth will cause significant particle loss; however, even collective vertical amplitude growth without loss will raise the vertical beam emittance and result in luminosity degradation.

\subsection{SIMULATION RESULTS}

The tracking program Evol was used for all simulations, using the B0D0 lattice described in the previous section. In order to drive the 5 th order resonance strongly for the worst-case scenario, a small $.1 \sigma$ beam-beam offset was included; closed orbit alignment errors of this magnitude at the collision points are quite possible. Tracking this lattice with no tune modulation with the beam-beam tuneshift given in Table 1 on the $Q_{x}=20.6$ resonance finds an island tune of $Q_{I}=1.51 \cdot 10^{-3}$. Since the synchrotron frequency of the Tevatron at this energy is approximately $Q_{M}=7.8 \cdot 10^{-4}$ (with a period $T_{M}=1 / Q_{M}=1280$ turns), the chaotic region of the tune modulation parameter space is quite accessible for moderate tune modulation depths $q$.

Tracking was performed with tune modulation depth $q=0.0010$, present only in the horizontal plane for comparison to the results originating in the similarly modulated Hamiltonian of Equation (7.1). This tune modulation amplitude corresponds to a horizontal chromaticity of about 3 units with a momentum offset 

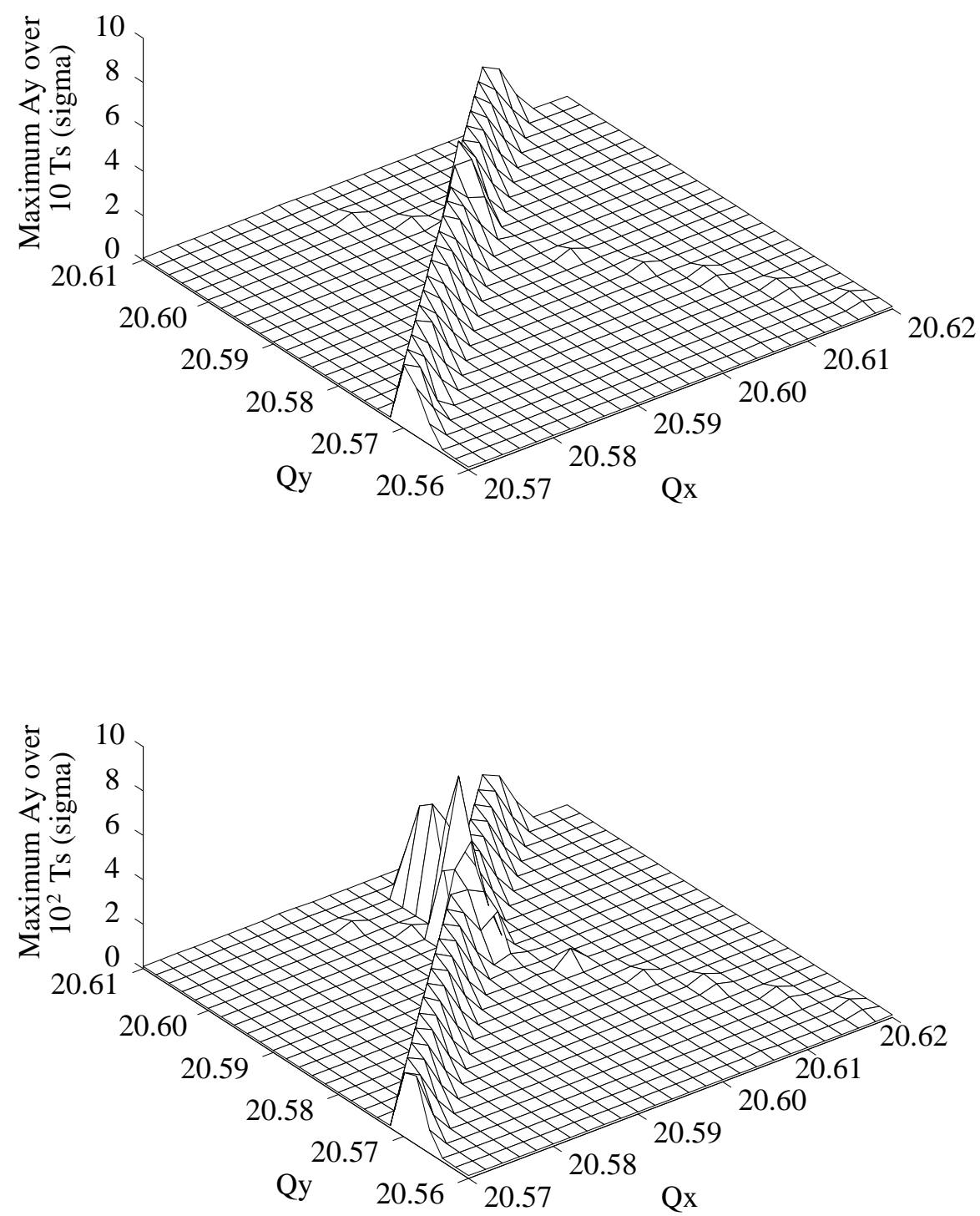

Figure 7.4: Maximum vertical amplitudes of single particles with initial vertical amplitudes $.1 \sigma$, tracked over 10 and 100 synchrotron periods. The particle's horizontal amplitude is $3 \sigma$, inside a chaotic band. 

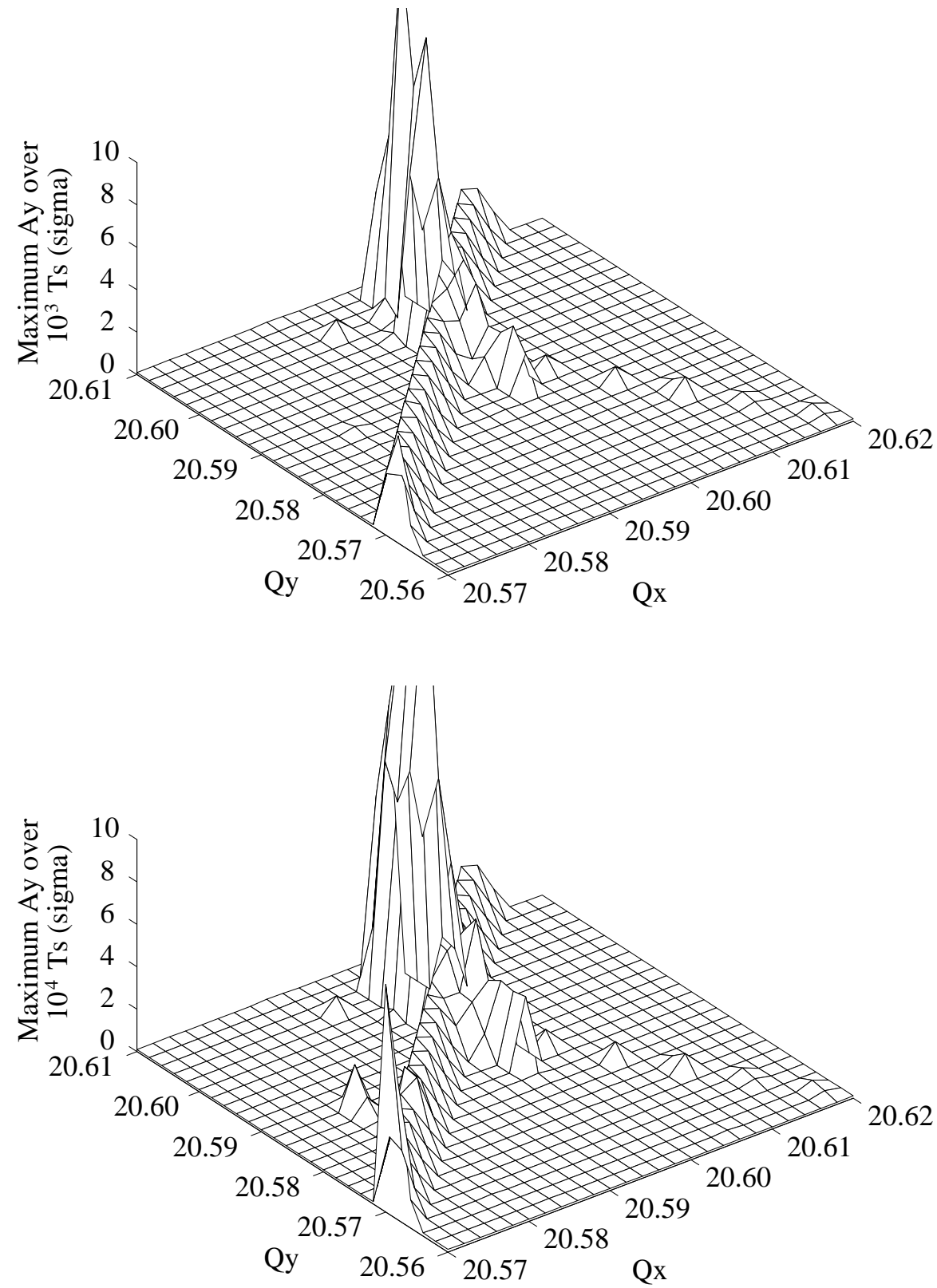

Figure 7.5: Maximum vertical amplitudes of single particles with initial vertical amplitudes $.1 \sigma$, tracked over $10^{3}$ and $10^{4}$ synchrotron periods. Conditions for tracking are otherwise the same as those in Figure (7.4). 
$\Delta p / p$ of $3 \cdot 10^{-4}$, realistic values for the Tevatron. Particles were launched with horizontal amplitude of $3 \sigma$, with base tune $Q_{x 0}=20.597$, and vertical amplitude of $.1 \sigma$ with various base tunes. Tracking was stopped when a finite number of synchrotron periods had been tracked $\left(10^{4}\right.$, corresponding to nearly 5 minutes of real particle evolution), or the vertical amplitude had reached $1.0 \sigma$. The one sigma vertical cutoff was introduced because the influence of the vertical motion on the horizontal stochastic band was expected to become non-negligible at moderate vertical amplitudes.

To establish the timescales of the relevant amplitude growth mechanisms, the maximum vertical amplitude was recorded for single particles launched at the above initial conditions over a mesh on the tune plane, for tracking times ranging from 10 to $10^{4}$ synchrotron periods. The tune mesh limits used were the same as those shown in the tune plane diagram, Figure (7.3), and the results of this tracking are shown in Figures (7.4) and (7.5). In these figures there is a quite definite amplitude growth near the intersections of the $Q_{x}-Q_{y}$ and $5 Q_{x}$ resonances that evolves over timescales of thousands of synchrotron periods, consistent with the naive timescales of modulational diffusion. Such growth is completely absent with modulation turned off $(q=0)$, where only amplitude growth on the $Q_{x}-Q_{y}$ resonance is seen due to energy exchange between the unbalanced horizontal and vertical amplitudes; this is a conclusive indication that the modulation drives this vertical amplitude growth. The growth also displays structure along the horizontal resonance, consistent with the modulational diffusion expectations of the dependence of the amplitude growth rate on distance from the nearest coupling resonance. There is also some growth that appears on the $3 Q_{x}+2 Q_{y}$ resonance; however, the structure along this resonance is quite minimal in comparison to the growth near the previously mentioned intersection of $Q_{x}-Q_{y}$ and $5 Q_{x}$ resonances.

Once the timescales of amplitude growth have been established, there remains 
the question of how the vertical amplitude evolves with time. It has already been mentioned that classical diffusion predicts that the vertical amplitude will grow proportionally to $t^{1 / 2}$ - if this is the case a plot of $\log a_{y}$ versus $\log t$ should be a straight line with a slope of one half. However, if the vertical amplitude grows exponentially with time,

$$
a_{y}(t)=a_{y 0} e^{\gamma t}
$$

the plot of $\log a_{y}$ versus $t$, not $\log t$, should grow linearly, and the slope of this line is the exponential growth rate $\gamma \cdot \gamma$ has units of inverse synchrotron periods, because the natural time unit for problems involving direct modulation is the modulation period, not turns.

Figure (7.6) shows three examples of vertical amplitude evolution over relatively long timescales, each plotted on log-linear and $\log -\log$ scales. It is clear from examining these evolutions (as well as those of many other particles at different distances $\alpha$ from the nearby weak coupling resonance $4 Q_{x}+Q_{y}$ ) that the vertical amplitude is growing as an exponential of time, not a power law as one would expect from standard diffusion phenomenology. It has been suggested that this growth may be explained by the dependence of resonance strengths on particle amplitude - the change in amplitude creates a changing resonance strength which feeds back upon the amplitude growth, creating exponential growth.

The exponential growth coefficient $\gamma$ can now be plotted versus the scaled distance to the weak coupling resonance as one varies the vertical tune along the $5 Q_{x}$ resonance to investigate whether there is any structure present. Since $\gamma$ is expected to vary over many orders of magnitude, we instead plot $\log \gamma$ versus $\alpha$; $\alpha$ can be directly determined from the vertical base tune $Q_{y 0}$ via

$$
\alpha=\frac{20.60-Q_{y 0}}{5 q}
$$

when considering the $4 Q_{x}+Q_{y}$ resonance to be the source of weak coupling. 

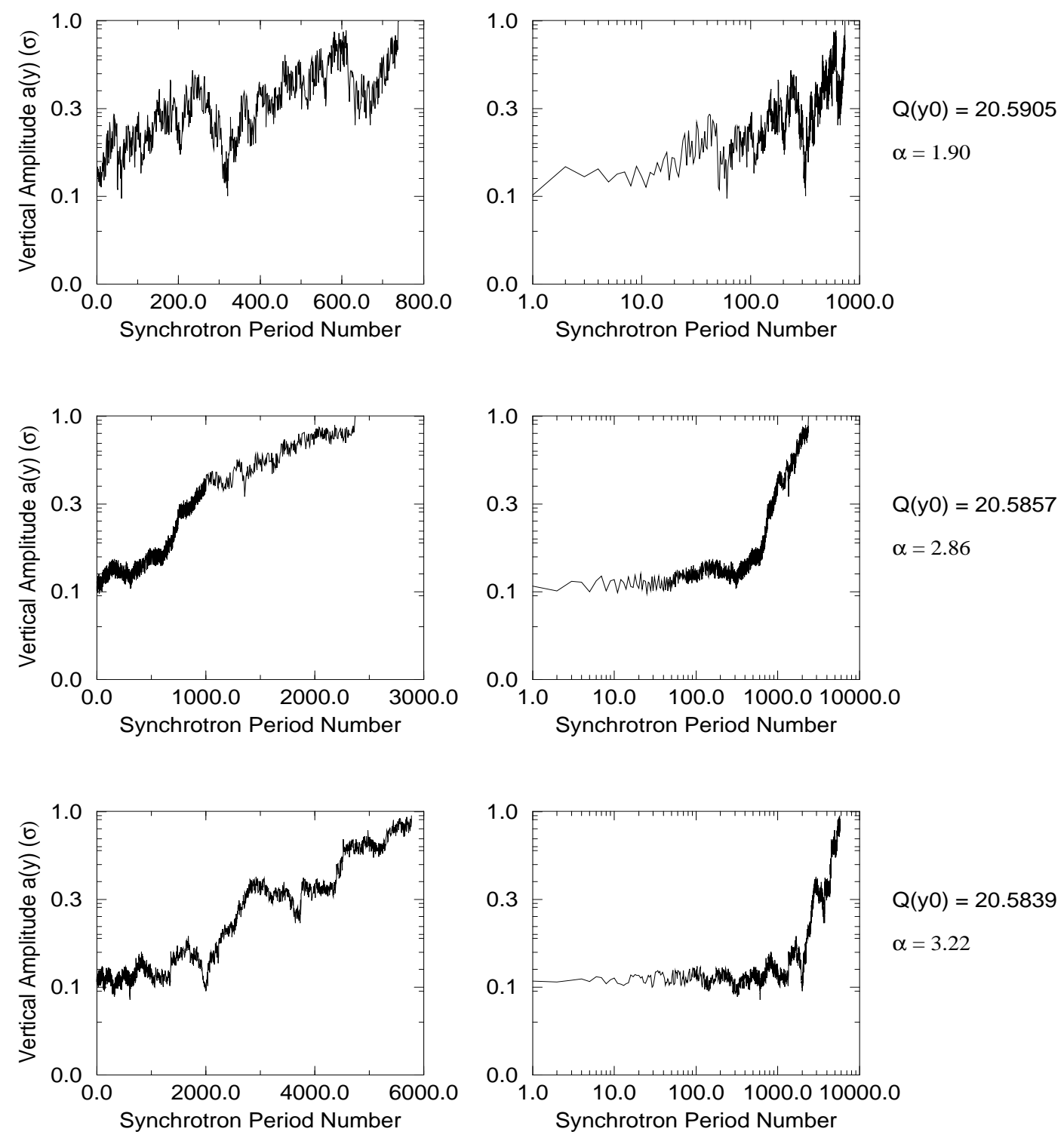

Figure 7.6: Character of vertical amplitude growth for particles launched within a horizontal stochastic band, and initial vertical amplitudes $.1 \sigma$ and three different base tunes, or three values of the scaled coupling resonance proximity $\alpha$. Tracking was stopped when the vertical amplitude reached $1 \sigma$ or after $10^{4}$ synchrotron periods. The vertical amplitude growth rate changes by an order of magnitude here. 


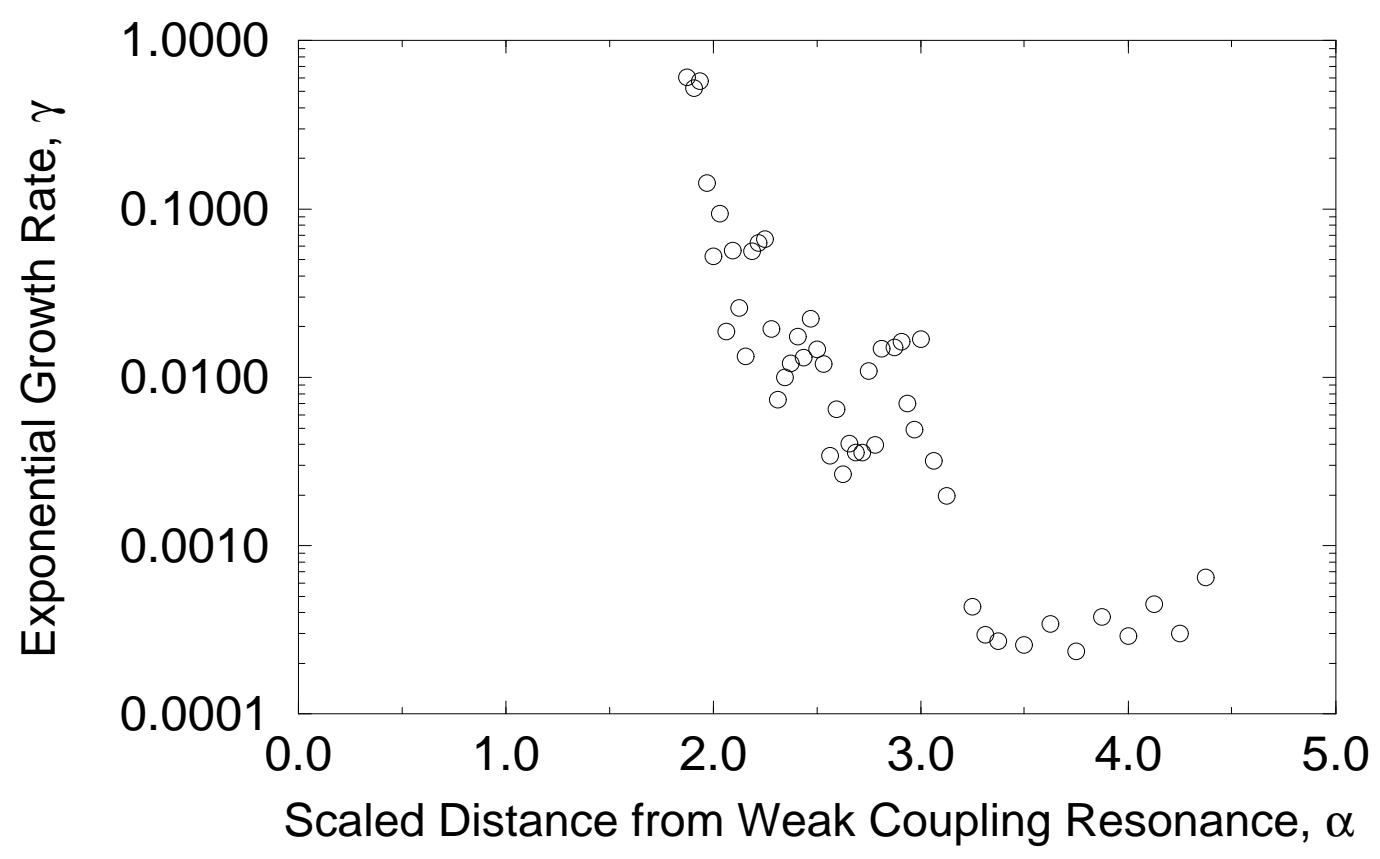

Figure 7.7: Exponential vertical amplitude growth rate $\gamma$ plotted versus the scaled distance $\alpha$ from the $4 Q_{x}+Q_{y}$ resonance.

Other resonances, such as $Q_{x}-Q_{y}$ and $3 Q_{x}+2 Q_{y}$ are also nearby, but are farther away in horizontal tune distance $\alpha$ than this resonance as can be seen in Figure (7.3). $\gamma$ is measured from a standard linear fit of tracked $\log a_{y}$ versus time data. Figure (7.7) shows this data; note the two distinct "plateaus" and the sudden drops in the growth rate at $\alpha=2$ and $\alpha=3$.

\subsection{CONCLUSIONS AND FUTURE DIRECTIONS}

Modulational diffusion has been investigated within a simple model of the beambeam interaction in the Fermilab Tevatron collider. Realistic operational parameters indicate that particles subject to horizontal stochasticity, or naively those that are within the tune modulation depth distance in horizontal tune of the $5 Q_{x}$ resonance, experience modulational diffusion that causes their vertical amplitudes to grow exponentially over timescales of thousands of synchrotron periods, or 
millions of turns, leading to possible long-term particle loss. The rate of this amplitude growth is also dependent on proximity of nearby coupling resonances, and shows a structural dependence similar to those of previous modulational diffusion studies, even though the vertical amplitude growth is not root-time as naively predicted in these models where resonance strengths are not action-dependent.

Under current operating conditions, no particles are expected to be affected by the $5 Q_{x}$ resonance this severely unless the horizontal tune drifts upwards, dragging particles into the fifth, or the linear beam-beam tune shift $\xi$ increases. However, with future luminosity upgrades, this tune shift per crossing will almost certainly rise and the operational space used in past runs may not be large enough to accommodate the entire tune spread of the beam. A significant portion of the beam would be influenced by the horizontal 7 th and 5 th integer resonances and vertical beam blowup could possibly occur. This circumstance would lead to luminosity degradation and intensity loss over a collider store as the beam size grows. These effects, were they present in an actual collider, would be difficult to diagnose due to their slow growth timescales.

Future studies should be twofold. First, a concrete theoretical structure of modulational diffusion should be investigated to conclusively show that in the case of amplitude-dependent coupling resonance strengths, vertical amplitude growth is exponential instead of root-time as in classical models. Second, the collective nature of the particle growth should be investigated to see what observable effects such a mechanism could have on the beam size (and thus luminosity) evolution over time. These collective effects and the emittance growth timescale dependence on proximity to driving resonances would be experimentally observable.

The octupole-decapole tracking model might also be used to investigate the amplitude growth mechanism once a theoretical framework is in place, to avoid the rather complex detuning and coupling of the beam-beam force. Tracking with 
this lattice has several distinct advantages - motion at large particle amplitudes is no longer stable, so no ad hoc aperture needs to be introduced, and parameters for a Hamiltonian description as in Equation (7.1) can easily be found to first order in the individual magnet strengths as described in Chapter 3. 


\section{CHAPTER 8 CONCLUDING REMARKS}

In this dissertation we have described one and two-dimensional nonlinear resonances in an accelerator context and examined how motion within a one-dimensional resonance may be perturbed by tune and beta function modulation. Tune modulation was used in an experiment, E778, to measure a quantity, the island tune $Q_{\mathrm{I}}$, associated with the strength of a one-dimensional resonance, showing excellent agreement with theory and simulation. This modulation also drives a nonuniversal instability, modulational diffusion, in weakly coupled systems, and this was investigated for a simple operational model of the Fermilab Tevatron collider.

Nonlinearities are always present in any accelerator, either deliberately installed or due to imperfections in magnetic field quality. A simple class of nonlinear perturbations, one-dimensional resonances, were the primary focus of this dissertation. They were shown to create structures, "resonance islands", within phase space, and capture of particles within these islands was demonstrated through simulation and experiment, creating coherent motion or "persistent signals" oscillating exactly at the resonant tune. Motion within these resonance islands was examined in a discrete Hamiltonian formalism and shown to be equivalent to that of a free pendulum. This motion was also parameterized by three quantities: the island tune, the phase space amplitude of the resonance and the resonance amplitude width.

A stability model was developed for particles oscillating close to the resonance island fixed point under the influence of tune modulation and beta modulation. This model predicts boundaries for the phase-locked stability of this motion that 
depend only on the island tune $Q_{\mathrm{I}}$ and the modulation strength and frequency. Comparison of the two showed that for realistic operational parameters the effects of tune modulation are much stronger than those of beta modulation, and both were compared to a simple robust simulation program to show excellent agreement for a particular resonance of interest.

The procedure and results of a tune modulation experiment, a portion of experiment E778 at Fermilab, were also described. The behavior of particles trapped in a nonlinear one-dimensional resonance, the $5 Q_{x}$ resonance at the horizontal tune $Q_{x}=20.40$, was systematically examined under the influence of controlled tune modulation for two distinct nonlinear configurations and three different horizontal island amplitudes. For one particular case of sextupole configuration and island amplitudes a detailed analysis of the response of the persistent signal at high frequencies agreed with the one-dimensional tune modulation model.

Frequency domain analysis was shown to be a useful tool for investigation of one-dimensional persistent signals, requiring no scaling with initial beam intensity or normalization of the beam position monitor measurement. This method has several advantages over time domain analysis, because tune modulation parameters and the island tune are all natural frequency domain variables characterizing this system. Because the tune modulation frequency can be finely controlled, this method may also allow investigation of the frequency (or particle) distribution within the nonlinear resonance island from examination of the rate of captured signal loss as the modulation frequency is increased.

The unperturbed single-resonance model was extended to two transverse dimensions, and a first order nonlinear model with two crossing resonances was examined. Simulation showed the existence of two-dimensional coherent motion and resonance islands at a particular set of base tunes corresponding to the point where the resonances under investigation crossed. The corresponding theoretical 
model predicted fixed point locations and phases accurately, but did not reproduce island tunes even at small nonlinear strengths. This indicates that the simple two-resonance model breaks down at the points in phase space where these resonances cross. Although persistent signals are present here, it is more likely that valuable information about the strengths of two-dimensional resonances will be obtained from two-dimensional smear, which is a qualitative measure of how the resonance distorts nearby nonresonant phase space.

An understanding of the effects of modulations leads to characteristic timescales of motion that are much larger than those typically encountered in the commissioning of many storage rings and colliders. Modulational diffusion is a previously uninvestigated amplitude growth mechanism, and it is shown here to cause luminosity loss over timescales of minutes using realistic operational parameters for the Tevatron. These timescales are long enough to prove difficult to diagnose yet short enough to significantly impact the luminosity lifetime of a collider, reducing the effectiveness of this machine in meeting experimental goals.

The character of amplitude growth due to modulational diffusion was shown to be different than that predicted by classical models, with exponential growth instead of root-time. Although not shown here, this is a general characteristic of systems where the resonance strength depends on particle amplitude, creating a feedback of this strength on the amplitude growth it creates. Modulational diffusion is not universal - there are limits that can be prescribed, corresponding to the tune modulation diagram, which preclude the existence of this mechanism in operational circumstance. These conditions can provide useful design limits for future colliders, including limits on power supply ripple and synchrotron frequency.

Future avenues of investigation and research include examination of the effects of tune modulation on two-dimensional nonlinear resonances. Though this is a more realistic scenario, the coupled nature of the motion and the complexity of 
examining multi-resonance systems make such an examination difficult. Since tune modulation exists in both planes of motion, it is feasible that such a mechanism could create a web of weak overlapping resonances in the tune plane, and that regions where modulational diffusion exists could be characterized. 


\section{BIBLIOGRAPHY}

Abromowitz, M. , and I. Stegun, eds. 1965. Handbook of Mathematical Functions. New York: Dover Books.

Byrd, J. 1992. Ph.D. Thesis, Cornell University, Ithaca, New York. (Unpublished)

Chao, A. and M. Month 1974. Nucl. Instr. Meth. 121, 129.

Chao, A. et. al. 1987a. SSC Labs Publication SSC-N-360.

Chao, A. et. al. 1987b. Phys. Rev. Lett. 61, 2752-5.

Chen, T. 1990. SSC Labs Publication SSC-323.

Chen, T. et. al. 1992. Phys. Rev. Lett. 68, 33-6.

Chirikov, B.V. 1979. Phys. Rep. 52, 265.

Chirikov, B.V. et. al. 1985. Physica 14D, 289-304.

Courant, E.D. and H.S. Snyder 1958. Ann. of Phys. 3, 1-48.

Edwards, D.A. and M.J. Syphers 1988. AIP Conf. Proc. No. 184, 2-189.

Gabella, W.E. 1991. Ph.D. Thesis, University of Colorado, Boulder, Colorado. (Unpublished)

Gabella, W.E. et. al. 1992. Fermilab Publication TM-1783.

Goldstein, H. 1980. Classical Mechanics. Reading: Addison-Wesley.

Grote, H. and F. Christoph Iselin 1990. CERN Preprint SL/90-13.

Holmes, S.D. 1991. Proceedings of the IEEE Particle Accelerator Conference, $2986-8$.

Holt, J.A., L. Michelotti and T. Satogata 1992. Fermilab Publication 537.

Landa11, L.D. and E.M. Lifshitz 1975. Mechanics. Oxford: Pergamon Press.

Lee, S.Y. et. al. 1991. Phys. Rev. Lett. 67, 3768-71.

Li, M.Y. 1990. Ph.D. Thesis, University of Houston, Houston, Texas. (Unpublished) 
Lichtenberg, A.J. and M.A. Lieberman 1983. Regular and Stochastic Motion. New York: Springer-Verlag.

Li1, J.Y. 1989. Master's Thesis, University of Wisconsin, Madison, Wisconsin. (Unpublished)

McLachlan, N.W. 1951. Theory and Application of Mathien Functions. London: Oxford Press.

Merminga, N. 1989. Ph.D. Thesis, University of Michigan, Ann Arbor, Michigan. (Unpublished)

Merminga, N. and K.Y. Ng 1989. Fermilab Publication FN-493.

Merminga, N. and K.Y. Ng 1992. Fermilab Publication FN-506.

Michelotti, L. 1986a. AIP Conf. Proc. No. 153, 236-87.

Michelotti, L. 1986b. Part. Accel. 19, 205-210.

Michelotti, L. 1991. Proceedings of the IEEE Particle Accelerator Conference, 1881-1883.

Peggs, S. 1982. Part. Accel. 12, 219.

Peggs, S. 1985. Part. Accel. 17, 11-50.

Peggs, S. 1988. SSC Labs Publication SSC-175.

Peggs, S. and R. Talman 1986. Ann. Rev. Nucl. Part. Sci. 36, 287-325.

Peggs, S. R. Talman and C. Saltmarsh 1987. SSC Labs Publication SSC-169.

Sands, M. 1970. SLAC Publication SLAC-121.

Saritepe, S. and S. Peggs 1991. Proceedings of the IEEE Particle Accelerator Conference, 473-5.

Satogata, T. and S. Peggs 1991. Proceedings of the IEEE Particle Accelerator Conference, 476-8.

Satogata, T. et. al. 1992. Phys. Rev. Lett 68, 1838-41.

Schachinger, L. and R. Talman 1985.

Schoch, A. 1958. CERN Technical Note 57-21. 
Tsironis, G. 1990. Fermilab AP Note 90-001.

Tsironis, G., S. Peggs and T. Chen 1990. Proc. of the Second European Particle Accelerator Conference, Vol. 2, 1753-5.

Vivaldi, F. 1984. Rev. Mod. Phys. 56, 737-55. 